IDENTIFYING AND CLASSIFYING SLUM AREAS USING REMOTE SENSING

Divyani Kohli 
Examining committee:

Prof.dr.ir. M. Boon

Prof.dr.ir. M.F.A.M. van Maarseveen

University of Twente

Universityof Twente

Prof.dr. M. Herold

Wageningen University

Prof.dr. C. Jürgens

University Bochum, Germany

ITC dissertation number 279

ITC, P.O. Box 217, 7500 AE Enschede, The Netherlands

ISBN 978-90-365-4008-7

DOI $10.3990 / 1.9789036540087$

Cover designed by Job Duim

Printed by ITC Printing Department

Copyright (c) 2015 Divyani Kohli

All rights reserved. No part of this publication may be reproduced without the prior written permission of the author.

DU FACULTY OF GEO-INFORMATION SCIENCE AND EARTH OBSERVATION 


\title{
IDENTIFYING AND CLASSIFYING SLUM AREAS USING REMOTE SENSING
}

\author{
DISSERTATION
}

to obtain

the degree of doctor at the University of Twente, on the authority of the rector magnificus, prof.dr. H. Brinksma,

on account of the decision of the graduation committee,

to be publicly defended

on Wednesday, November 25, 2015 at 14:45

by

Divyani Kohli

born on September 16, 1981

in Dehradun, India 
This thesis is approved by:

Prof.dr.ir. Alfred Stein (promoter)

Dr. Richard Sliuzas (co-promoter)

Dr. Norman Kerle (co-promotor) 
To my Oapu, Ojju Singh

Thank you for being my inspiration 



\section{Acknowledgements}

My final but most important task is to acknowledge all those people that have contributed in the culmination of this thesis. Though it is nearly an impossible task to thank everybody who was directly or indirectly involved, I will try my best. It has been a considerably long journey, full of personal and scientific experiences that have enriched my life. A journey, with lots of ups and downs where there were moments of satisfaction/happiness, but also of frustration and despair. It has not been a journey of scientific investigation alone but also of self-discovery where I identified my strengths, weaknesses and learnt to be at peace with myself. So, to start with, I would like to thank this journey for the opportunity to delve into the ocean of knowledge and also learn about amazing scientific innovations.

I feel blessed to have a supervisory team that stood by me all these years. Prof. Alfred Stein and Dr. Richard Sliuzas, this thesis would not be possible without your guidance. I have no words to thank you for your endless support. Alfred, you have been a source of inspiration for me. Your impeccable time management, dedication to science and student's progress is so inspirational. There was never a day when you were unavailable to help, never an unanswered email. Your prompt responses and critical feedbacks and, at times, the much needed push helped me finish chapter drafts. Too often, I have felt guilty about not being able to keep the pace. Among other things, I have also learnt to appreciate and report on research outputs, that I always assumed "not too significant". I still remember the metaphors you used to quoteparticularly the one between writing a research paper and composing music for an Opera.

Richard, you were my strongest support while I was in Netherlands and also in India. I knew I could just walk into your office anytime without prior appointments. Many times, over the past years, I have talked/written about various problems and challenges- both professional and personal. You have always offered unconditional support, advice and help. I was introduced to slum-related research by you, because of which I started to see the world with a completely different perspective. I must admit that I was also one of those who would move around with a blind eye towards the "invisible homes". Thank you, for helping me broaden my perspective as well as for the patience, scientific insights, brilliant ideas and feedback during the endless exchange of chapter drafts. Your advice and emphasis on scientific details but "to the point" writing style is something I will try to adhere to. Please bear my long, narrative style in this section at least.

I sincerely extend my appreciation for Dr. Norman Kerle. Your scientific brilliance and "out of box" thinking has always inspired me. Though, we have 
had little correspondence over the past two years, I feel enriched from the discussions and feedbacks of the initial years. Many times, I have delved into the discussion notes, your comments and meeting-recordings to evaluate and improve my work.

I am thankful to the many people I met during field-work. Somewhat new to the domain to slum-related research, it has been an eye-opening experience. It was a stark face-off with human misery but also with hope and optimism. The numerous interactions surely gave me a realistic version of all the theory I had read or at times, misinterpret. These interactions, no doubt, helped me pursue my university's motto: 'High tech, human touch'. Some people, of course, deserve a special mention. I deeply appreciate Sulochana mam's help during Pune field-work. My thanks to Anjana Vyas and Dhruv from CEPT, Pratibha Joshi of Shelter associates and Sheela Patel of Sparc for useful discussions. Special thanks to Sharad Mahajan of MASHAL for sharing Pune slum atlas. I also learnt a lot during my field-work to Kisumu, Kenya. My deep gratitude to Ben Obera, Margaret, Patrick, Robert, Soloman, Evans, Merciline, Vincent, Emmanuel, Malachi and Matthew for useful discussions.

I extend deep appreciation to the GeoEye foundation for providing images for this research. Thanks to Peter Hofmann from University of Salzburg for sharing valuable research ideas. Big thanks to the ever youthful, David Rossitier, for the numerous conversations and advices on various issues. Special thanks to Prashant sir for brain-storming sessions and brilliant ideas; Lesslie sir, for providing technical ideas. Thanks to Wietske Bijker for being a great supervisor during my Masters and for discussions during PhD proposal formulation. Thanks also to Mr. P.L.N Raju and Dr.Y.V.N. Krishna Murthy for helping me work from Indian Institute of Remote Sensing (IIRS) in Dehradun.

I feel indebted to the European Commission's Erasmus Mundus program for the scholarship to carry out research at ITC. Prof. Martin van Maarseveen was my first contact here, thank you for introducing me to the right people. Thanks to Monika, Emile, Javier, Johannes, Mark, Liza, Arvind and Frans for being such nice PGM colleagues. I am deeply grateful to Loes Colenbrander for assisting diligently in every PhD-related issue, Marion Pierik for coordinating the financial issues, Paul van Dijk for listening to our woes patiently, Theresa van den Boogaard for meticulously handling the bureaucratic issues and Marga Koelen and Carla Gerritsen for helping in the library. I appreciate the IT-helpdesk for solving all IT problems. A big thank you Job Duim for working on the cover page. Thanks to the cheerful Petra Weber for helping with various issues.

Being at ITC is a unique experience given the different nationalities and cultural diversity of students. I feel fortunate to have friends who shared their ideas about various things, life experiences and of course, food! Maitreyi (birdy), 
Sejal and Priya, thank you for being a part of the family in NL. Birdy, thank you for the beautiful companionship and for standing by me. Sejal, you are my role-model, thanks for sharing your ideas on various subjects. Priya, thanks for all the support. Claudia Robbie Sliuter, Claudia U, Janik, Leo and Nelly, it was wonderful sharing Casa de Basil with you all. Mike McCall and Margaret, thank you for creating such a special place! Special thanks to Valentina Spanu for being an amazing friend. Fangfang and Fangyuan; thank you for enriching my life in many ways, especially for the various sport activities we participated in. Sincere thanks to my friends Sanaz Salati, Xuanmei, Flaviah, Armindo, Rafael, Pu Hao, Quang, Sukhad, Christine, Mike, Mareila, Parveen, Tanmoy, Debanjan Da, Anandita, Paresh bhai, Beti, Rehana, Sara and Adish for the enjoyable (and sometimes stressful!) times we shared together. Gaurav and Yamini, your place always felt like home. Zoya, Mitava and Devashree, I really cherish the moments we spent together in ITC. Thank you, Monika Lengoiboni and Anthony to help settling-in when I was nothing but a "confused student". My sincere thanks to Rohan Bennett and Nicholas Hamm for the friendship and interesting conversations. Nick, thank you for the brownies and cakes! Laura, Cynthia and Linda, it was a pleasure to stay at your place and interact with you. Razieh joon, we have shared the most stressful moments together and laughed through adversities. I feel so fortunate to share my office with you.

I feel like the luckiest person to have a great family. My parents have always supported my dreams and aspirations. I would like to thank my mom to be a source of inspiration and strength for all of us. My deep appreciation is extended to my sisters and brother-in-laws. Thank you Sona and Jiju for providing long-distance support, also in the form of recipes whenever I craved Indian food or had to cook for friends. Vikram and Mona, I knew that I could count on you for anything, anywhere and anytime. Thank you my soul-mate, Tina, and Sanjeev for the much-needed moral support. I thank you all for eternal love, support and blessings. I would like to thank the little chickos for bringing so much joy in our lives. I also thank Dolly aunty and Harjit uncle for hosting my stay at their beautiful house in Dehradun.

Finally, I express my deepest gratitude towards Pali aunty and Papu. Both of you have set an example of extraordinary life for me, where age does not fade away the passion of life! I have totally enjoyed and learnt a lot from our philosophical, spiritual, historical and political discussions. Papu, you have provided all the possible help and at times, arranged logistics to help me finish my PhD. Coming back to India meant working alone at home, where I missed intellectual exchanges and discussions with colleagues. You helped me with that void by intently listening to my research ideas and enriching me by your informal but very informed comments. Your curiosity for knowledge and immense understanding of various subjects is truly inspiring. Thank you for tolerating my R\&D (rona \& dhona) and for having implicit confidence in me. 


\section{Table of Contents}

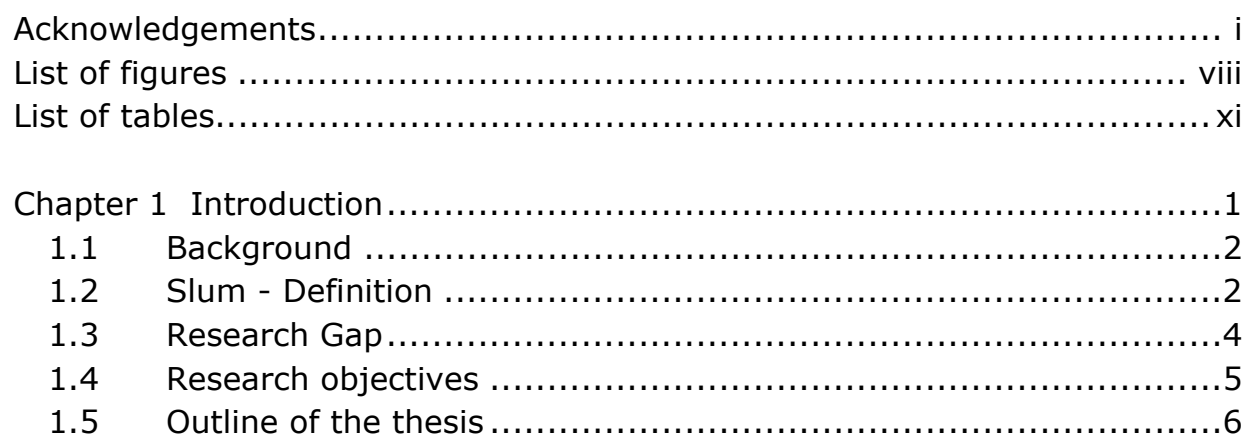

Chapter 2 An ontology of slums for image-based classification ...............9

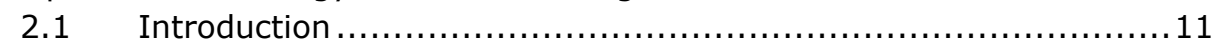

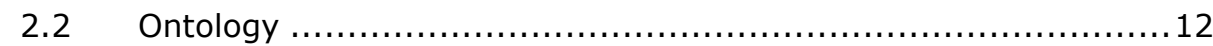

2.3 Previous work on slum identification and mapping ................. 13

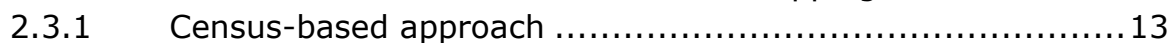

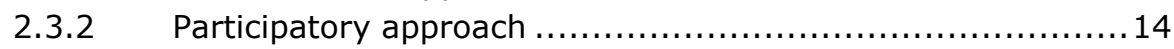

2.3.3 Remote sensing based approach........................... 15

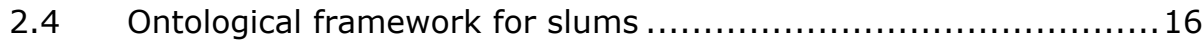

2.4.1 Specification ............................................... 16

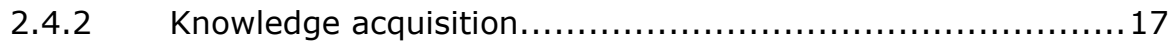

2.4.3 Conceptualization .............................................. 19

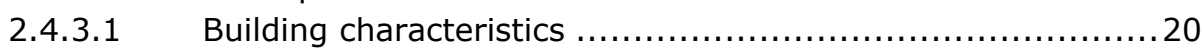

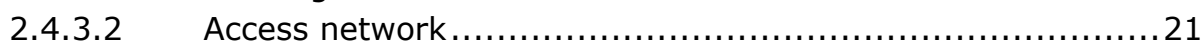

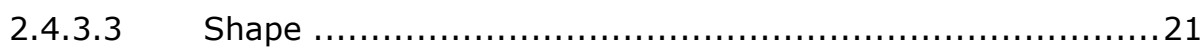

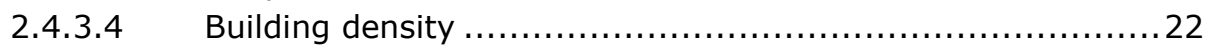

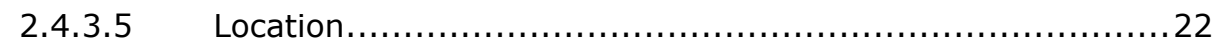

2.4.3.6 Neighborhood characteristics ................................. 24

2.4.4 Implementation- local adaptation of GSO $\ldots \ldots \ldots \ldots \ldots \ldots \ldots . \ldots 24$

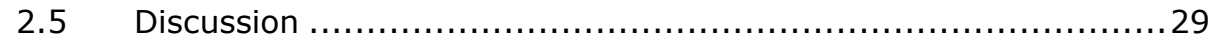

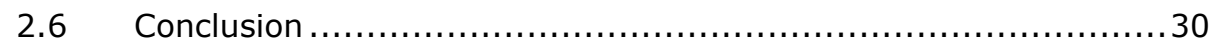

Chapter 3 Transferability of Object-Oriented Image Analysis Methods for

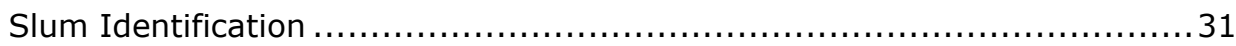

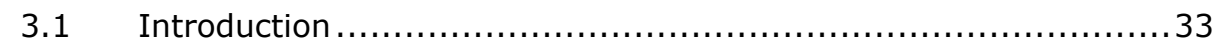

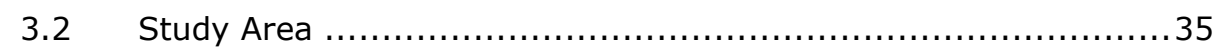

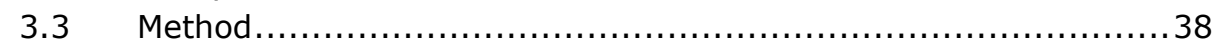

3.3.1 Local Ontology of Slums in Ahmedabad ......................... 38

3.3.2 Converting Local Ontology to OOA Parameters .................40 40

3.3.3 Approach for Slum Identification and Classification............43 43

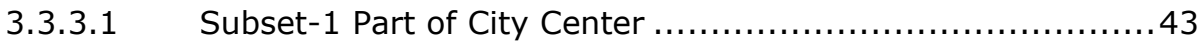

3.3.3.2 Subset-2 Full City Center ................................... 44 
3.3.3.3 Subset-3 Residential and Industrial Part of Outer City.........44

3.3.3.4 Clean-Up ....................................................... 45

3.3.3.5 Accuracy Assessment........................................... 46

3.3.3.6 Identifying the Most Stable Parameters ......................... 46

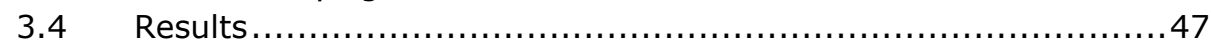

3.4.1 Identifying the Most Stable Parameters .........................49 49

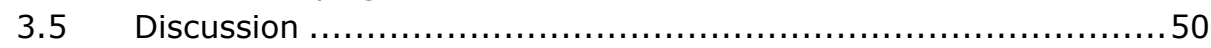

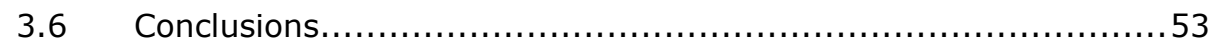

Chapter 4 Urban slum detection using texture and spatial metrics derived

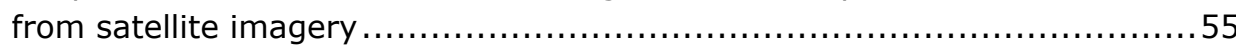

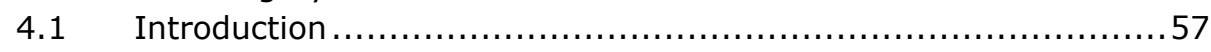

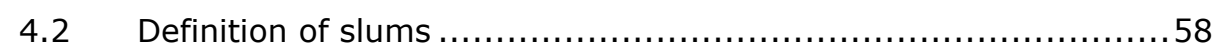

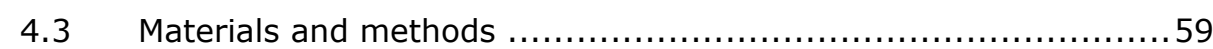

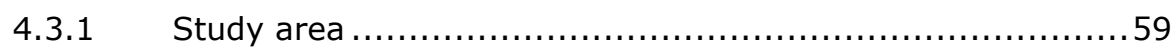

4.3.2 Image data ...................................................... 60

4.3.3 Local ontology of slums in Pune.................................60

4.3.4 Conversion of local ontology to OOA parameters...............62

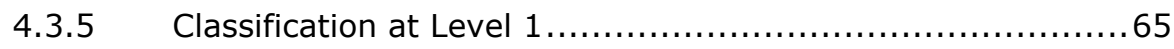

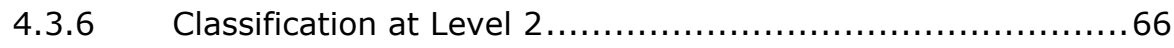

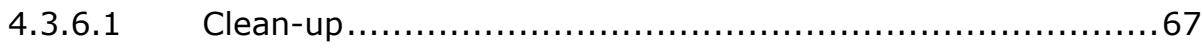

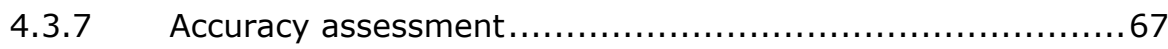

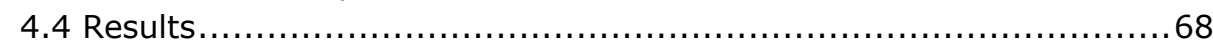

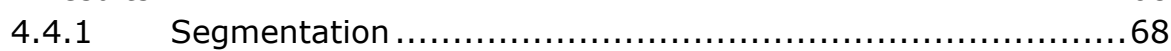

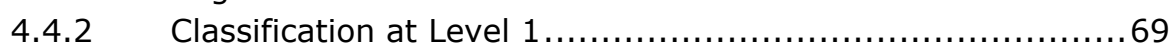

4.4.3 Classification of slums......................................... 70

4.4.4 Accuracy assessment........................................ 71

4.4.4.1 Accuracy of basic land cover features ........................... 71

4.4.4.2 Accuracy of classified slums ..................................... 72

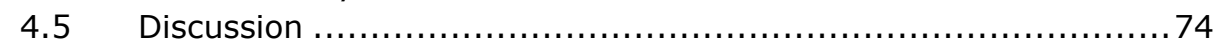

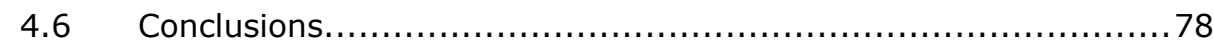

Chapter 5 Uncertainty analysis for image interpretations of urban slums ... 79

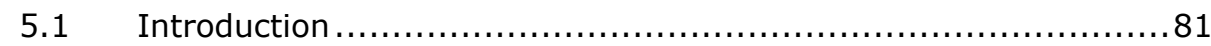

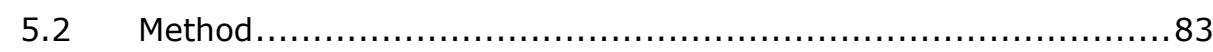

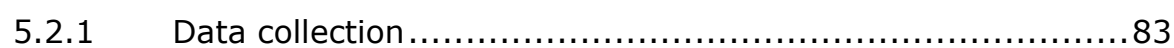

5.2.2 Data collection on image interpretation ........................ 83

$5.2 .3 \quad$ Selection of respondents ........................................ 84

$5.2 .4 \quad$ Statistical analysis.............................................. 85

5.2.5 Group-wise and city-wise comparison of the delineations .....88

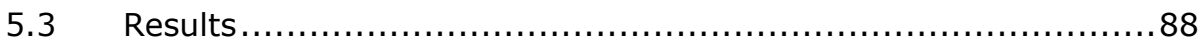

5.3.1 Area of maximum agreement level ........................... 88

5.3.2 Modelling uncertainty using random sets ....................... 90

5.3.3 Difference in delineations of the two groups and cities .........96 96 


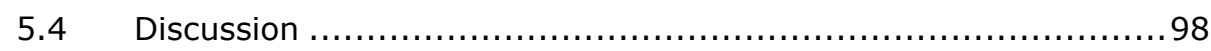

5.4.1 Factors used to distinguish slums from non-slum areas.......98

5.4.2 Factors leading to difficulties in slum interpretation ............. 98

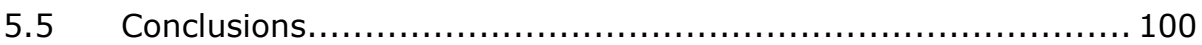

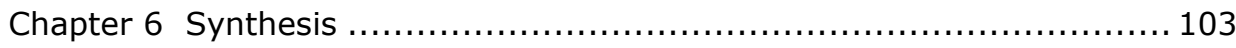

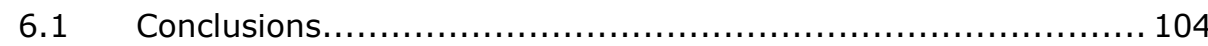

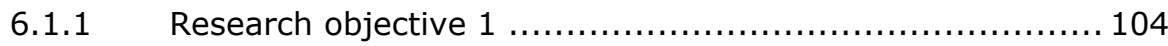

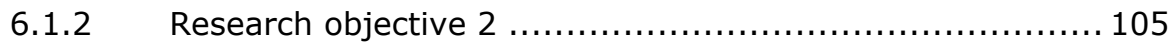

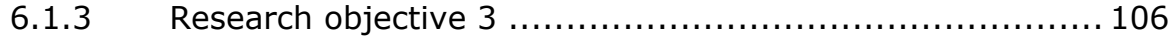

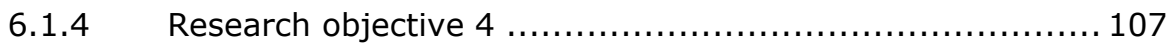

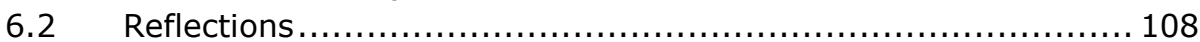

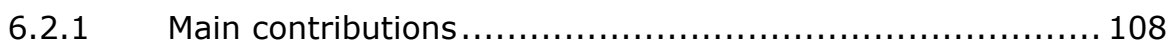

6.2.2 Recommendations for further research .................... 109

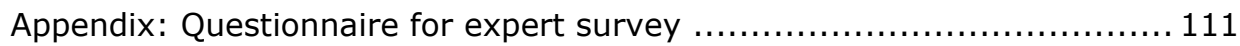

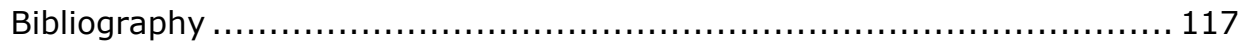

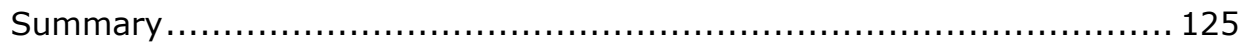

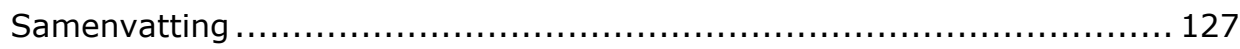

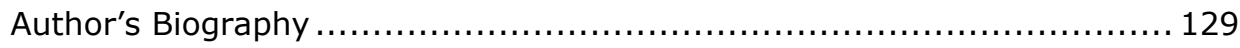

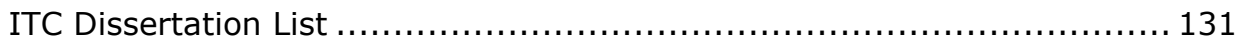




\section{List of figures}

Figure 2-1 An example of slum delineation in different contexts by an Indian expert. City names: a) Ahmedabad (India), b) Nairobi (Kenya), c) Cape Town (South Africa), d)Kisumu (Kenya). Source: Google Earth.

Figure 2-2 The six general indicators categorized to form a hierarchy to represent concepts at three spatial levels.

Figure 2-3 Diagram of building attributes with the corresponding values derived from the expert survey.

Figure 2-4 Diagram of access network characteristics observable via VHR images with the corresponding values derived from the expert survey. ......22

Figure 2-5 Diagram showing possible site conditions of a slum derived from expert survey.

Figure 2-6 Diagram of socio-economic factors that are possible driving forces for development of slums according to domain expert survey.

Figure 2-7 A snapshot of Kisumu, Kenya. The Nyalenda slum area is clearly visible below the major road running diagonally from bottom left to top right.

.25

Figure 2-8 Delineations of slums from a snapshot of Kisumu, Kenya produced by 5 randomly selected domain experts.................................... 26

Figure 3-1 Pan-sharpened GeoEye-1 image in true color composite display and ground photographs of different parts of the city: (a) Typical slum house made of non-durable materials. (b) A slum settlement on the east of the river. (c) Old City area. (d) Newly planned residential complexes emerging on the western side of the river. 36

Figure 3-2 Pan-sharpened GeoEye-1 image of Ahmedabad city in color infrared composite display. The three overlaid rectangles mark the extent of different subsets analysed in this study... 37

Figure 3-3 Multiresolution segmentation of the multispectral image ( $\psi=40$, Shape $w_{\text {shape }}=0.5, w_{\text {compt }}=0.5$ ). (a) The individual buildings and trees are outlined in the non-slum areas. (b) In the slum areas, image objects are clumps of buildings due to the relatively low color contrast.

Figure 3-4 Integrating expert knowledge from local ontology of Ahmedabad at settlement level. Slums tend to have an irregular shape with very high building densities in comparison to non-slums. .45

Figure 3-5 Slum delineations by an expert overlaid on Subset-2 and the magnified view of two such delineations: (a) A slum on the bank of the river (b) Slum buildings under the vegetation cover. 46 
Figure 3-6 Subset-1 classified using ruleset 1 . Slums form a strip close to the east of the river with the exception of one slum patch on the west.

Figure 3-7 Classification of Subset-2 with Ruleset-2 after applying clean-up procedures.

Figure 3-8 Classification results of Subset-3: (a) Application of Ruleset-1, industrial buildings are misclassified as slums due to their large roofs. (b) Application of Ruleset-3, the slum area is now correctly classified after removing the false positives.

Figure 4-1 Location map of the study area in the South-Western part of India. The ward map shows the administrative wards of Pune city. The pan-sharpened Quickbird image is from the central part of the city. 59

Figure 4-2 Slum delineations provided by a local NGO (MASHAL) displayed on the Quickbird image of Pune city. 61

Figure 4-3 Method linking texture layer and spatial metrics to GSO at object and settlement level. 65

Figure 4-4 Settlement-primitives at Level 2 using texture layer GLCM contrast: (a) Highly dense buildings in slums outlined and displaying a clear contrast to neighbouring segments; (b) Planned area with segments comprising different land cover features. 69

Figure 4-5 Classification of image at Level 1: (a) Magnified view of built-up classified in slum area; (b) Segmentation result of the same area. 70

Figure 4-6 Final classified map of the study area with slums, ground pictures and corresponding zoomed views of image: (a) Ground photograph of buildings in Old City; (b) Ground photograph of a typical slum settlement; (c) Magnified view of a part of Old city misclassified as slum on final map; (d) A typical slum settlement.

Figure 4-7 Figure showing source of error in classification resulting from: (a) generalization; (b) political/administrative definition; (c) vegetation cover; (b) false-positives from Old City. .73

Figure 5-1 Slum delineation in different contexts by a single expert in the cities of a) Ahmedabad (India), b) Nairobi (Kenya), c) Cape Town (South Africa). Source: Google Earth.... .85

Figure 5-2 Different forms of extensional uncertainty: 1) slum delineation encloses the actual slum boundary; 2 ) slum delineation inside the actual slum boundary; 3 ) slum delineation same as the actual slum boundary. 87

Figure 5-3 Agreement level layers of slum delineations by 19 experts for the three contexts (a) Ahmedabad (India), (b) Nairobi (Kenya) and (c) Cape Town (South Africa). .89 
Figure 5-4 The bootstrap estimates of random set for Nairobi: (a) the random set model shown by its covering function; (b) the standard deviation of random

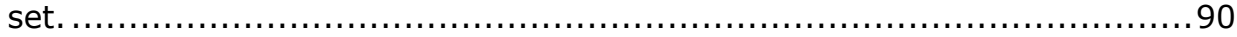

Figure 5-5 Overlay of respondents' slum interpretations for the snapshot of Nairobi and the magnified view of two areas: (a) boundary deviation due to generalization; (b) deviation resulting from inclusion/exclusion of fringe in Nairobi. 92

Figure 5-6 The bootstrap estimates of the random set for Ahmedabad: (a) the random set model shown by its covering function; (b) the standard deviation of random set. .93

Figure 5-7 Overlay of respondents' slum interpretations for the snapshot of Ahmedabad. 94

Figure 5-8 The bootstrap estimates of random set for Cape Town: (a) the random set model shown by its covering function; (b) the standard deviation of random set.

Figure 5-9 Overlay of respondents' slum interpretations for the snapshot of Cape Town and the zoomed-in view of two areas ( $a$ and $b$ ) showing boundary deviations resulting from inclusion or exclusion of vegetation. 96

Figure 5-10 Average, minimum, maximum and standard deviation of areas delineated by the advanced (light) and basic (dark) group for Nairobi, Ahmedabad and Cape Town... 


\section{List of tables}

Table 2-1 List of countries referred to by respondents in the expert survey grouped by region.

Table 2-2 Characteristics of slums for each general indicator that were specified by domain experts.

Table 2-3 Number and percentage of experts who referred to a general deprivation issue while defining slums. 18

Table 2-4 Number and percentage of experts identifying a site or neighborhood condition as being a likely characteristic of an Asian or SSA slum. 23

Table 2-5 Translation of visual observation and expert knowledge to imagebased indicators. 27

Table 2-6 Adaptation of ontological concepts from generic slum ontology to the case study and its respective OOA parameterization. 28

Table 3-1 Adaptation of concepts from the generic slum ontology to a local ontology for Ahmedabad followed by respective object-oriented image analysis (OOA) parameterization.

Table 3-2 The definitions of the stable parameters used in the classification of three subsets. For details on each of the parameters, refer to Haralick et al. (1973) and eCognition reference book (Trimble 2014). 41

Table 3-3 Ruleset summary and the quantitative classification criteria used for different subsets. The classification steps show the sequence with top being attempted first. 42

Table 3-4 Allocation of weights to the parameters depending on applicability in different subsets. 50

Table 4-1 Adaptation of GSO concepts to an ontology for Pune with OOA parameterization at environs, settlement and object levels. 62

Table 4-2 Definitions of the parameters used in the classification. For details, refer to Trimble (2014). 64

Table 4-3 Ruleset summary and the quantitative classification criteria with the sequence from top to bottom. 68

Table 4-4 The producer's and user's accuracies of the land cover classes at Level $1 ; \mathrm{PA}=$ producer accuracy, $\mathrm{UA}=$ user accuracy, $\mathrm{OA}=$ overall accuracy and Kappa = Kappa agreement index

Table 5-1 Number of respondents grouped based on their experience in remote sensing and slum mapping. 
Table 5-2 List of region of origin and countries referred to by respondents in

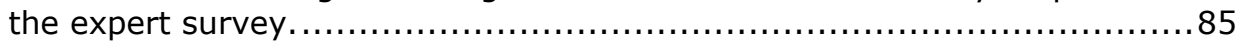

Table 5-3 Percentage of identified slum area at maximum agreement level. The percentage of adjusted area is the area after removing the over-estimated area identified by three or less respondents. 89

Table 5-4 Linear relations between area and the explanatory variables, with respect to different cities and experience...................................... 97

Table 5-5 ANOVA of area in relation to experience and cities within the

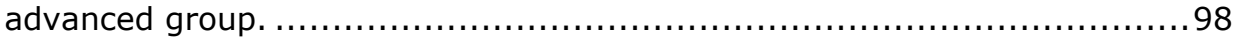


"I often look up there and wonder about the sky, stars, different shades, sun. and moon. There could be someane up there looking back at us wondering about us" 
Chapter 1

Introduction 


\subsection{Background}

More than half of the global population is urban now owing to unprecedented pace of urbanization in almost all the world regions (UN-HABITAT 2012). In addition to urban population growth, governments in developing countries face the challenge of high growth rates of poverty. This is manifested most conspicuously in the proliferation and expansion of slums that are home to a growing proportion of urban dwellers (Ooi and Phua 2007). One third of the global urban population lives in slums (UN-HABITAT 2014). To work towards the improvement of living conditions of slum-dwellers, a number of steps are being taken at national as well as international level. In particular, the Millennium Development Goals (MDGs) formulated by the United Nations (UN) recognize various problems related to poverty and development and have led to the demand for data on several key indicators, including the percentage of slum dwellers in all countries (UN-HABITAT 2003a).

This thesis explores the use of remote sensing (RS) methods that can assist in making the provision of data and information on slums more readily available. Recording of unidentified sections of urban poor by mapping unlisted and hidden slum clusters, other temporary settlements and infrastructure is an important strategy to potentially improve the lives of slum dwellers (Sen et al. 2003). Quantification of slum estimates by identifying and defining slums spatially in a consistent manner is important in geographical targeting within slum intervention programs on global as well as local scales (UN-HABITAT 2003b). The potential of RS to assist in producing statistics on slums is being realized by international experts; robust, systematic methods, however, do not exist currently (Sliuzas et al. 2008a). The major hurdles are different definitions across different contexts and the high variability in slum characteristics. Spatial statistical methods have never been considered so far.

\subsection{Slum - Definition}

Rapid urbanization, rural to urban migration, lack of affordable housing, increasing poverty and inequality are often cited as the main reasons for slum development and growth (UN-HABITAT 2003a). Although these reasons or their combination may vary for different cities of the world, slums tend to share some common features globally. A prominent feature is that slum areas tend to have a high concentration of poor people and poor shelter and physical environmental conditions (UN-HABITAT 2003c). Different countries often develop their own definitions for slums, thus making international comparisons and monitoring difficult. In order to work towards the goal of improvement in the lives of slum dwellers, a global definition of slums was formulated by an Expert Group Meeting in 2002 at Nairobi (UN-HABITAT 2003b). According to this definition, a slum household lacks in at least one of the following: 
- Secure tenure, i.e. the right of all individuals and groups to effective protection by the state against forced evictions.

- Access to safe water - A household is considered to have access to safe water supply if it has a sufficient amount of water for family use, at an affordable price, available to members without being subject to extreme effort, especially to women and children.

- Access to sanitation - A household is considered to have adequate access to sanitation if an excreta disposal system, either in the form of a private toilet or a public toilet shared with a reasonable number of people, is available.

- Durability of housing - A house is considered 'durable' if it is built on a non-hazardous location and has a structure, which is both permanent and enough to protect its inhabitants from the extremes of climatic conditions such as rain, heat, cold, humidity.

- Sufficient living area - A house is considered to provide a sufficient living area for the household members if not more than two people share the same room.

UN-HABITAT accentuates the fact that the above indicators convey an operational definition of a slum household that can be modified with the contextual information. A generalized definition for a slum area was also formulated as follows:

"[A slum is] a contiguous settlement where the inhabitants are characterized as having inadequate housing and basic services. A slum is often not recognized and addressed by the public authorities as an integral or equal part of the city" (UN-HABITAT 2003b).

A dwelling that has all of the following is considered to be durable, while slum dwellings often lack one or more these:

- Permanency of Structure - walls, roof and floor;

- Compliance with building codes;

- The dwelling is well maintained and structurally sound;

- The dwelling is not located on or near toxic waste;

- The dwelling is not located on a flood plain;

- The dwelling is not located on an unstable slope;

- The dwelling is not located in a dangerous right of way (e.g. rail, highway, airport, power lines and pipe lines). 
Several of the above criteria for durability can potentially be extracted from RS data or derived with the aid of such data.

\subsection{Research Gap}

Traditionally, urban slum and non-slum settlements have been differentiated based on administrative definitions or income-based indicators. Official data collection methods in most developing countries are typically head-count based (census) and do not provide detailed spatial information about the concentration or location of slum-dwellers. Previous work on slum mapping comprises of three major approaches: mapping census data with GIS (Baud et al. 2009, Baud et al. 2008), participatory methods, (Lemma et al. 2006, Joshi et al. 2002, Hasan 2006) as well as RS-based methods (Mason and Fraser 1998, Sliuzas et al. 2008b). While there is a clear advantage of using census data as it provides household level information, the long temporal gap between two census surveys and degree of data aggregation is problematic. A participatory approach is beneficial as it involves mapping the details of informal settlements at individual or household levels with the cooperation of slum-dwellers. A major challenge, though, is the amount of resources required for collecting such detailed data. Thus, alternative methods that can reduce enumeration resources and can systematically generate city-wide slum maps are sought. RS using very high resolution (VHR) images are potentially useful for that purpose.

The durability indicator of the UN-HABITAT slum definition is of particular interest for this thesis as it concerns physical aspects of slums that may be visible on RS images. RS can provide spatially disaggregated data of a high temporal consistency, which can facilitate rapid monitoring and effective intervention of slums by local authorities. A further development in standardised slum mapping methods is challenging due to the variability in slum characteristics and their definitions across the world and also diversity within a city (Sliuzas et al. 2008a). Among other reasons, this difference can be attributed to variation in building materials depending on local availability, different topographic conditions, cultures and architectural traditions. Slums often develop in areas that are disaster-prone such as steep slopes, floodprone and marshy areas, along railway tracks and close to major roads. These sites may be unattractive for planned development because of potential risks. The variety of slums around the world is also reflected in different names depending on geographical location and material used to build them - e.g. chawls in Mumbai, kachiabadi in Karachi, vijiji in Nairobi, favellas in Rio de Janiero or jhuggi jhopri in Delhi. Even within a single city, slums can differ in many ways. An example is the case of Cairo where slums on former agriculture lands have quite a regular structure based upon the former field patterns whereas slums on desert land lack such a pre-defined structure and are more 
chaotic. Furthermore, there is a need for methods to differentiate poverty areas or slums from slum-like areas (Angeles et al. 2009). Slum-like areas may share similar physical characteristics and appear to be slums, whereas they are essentially different from slums. Some historic city areas that have developed organically may share 'slum-like' features. These areas, for example, may exhibit very high building densities with irregular road network. Unacquaintance with such areas may lead to mis-interpretations. Automatic image interpretations can thus contain uncertainties that may negatively impact the accuracy of the final classified products. This issue also receives attention in this thesis using a novel spatial statistical approach.

VHR images are commonly available and can likely be used to extract detailed slum information better than ever before. Slum areas can be monitored at a higher frequency than before. Extraction techniques that were entirely pixelbased, have now been supplemented by object oriented image analysis (OOA) methods. In order to apply these, clear and unambiguous rulesets have to be developed and applied; these in turn rely on an appropriate definition of slums. In order to apply them, a careful treatment of spatial uncertainty and spatial data quality is required. Also, given the detail available in VHR images, the opportunities are coupled with higher levels of complexity in terms of extracting and representing real-world knowledge by means of RS. It is thus important to understand and integrate epistemological and ontological aspects of real-world objects for wider applicability of derivation methods using remote sensing (Blaschke 2010). Previous research on RS-based slum mapping relied mainly on complimentary ground information, or is methodologically limited to the study-area in which the research was carried out. Generically applicable RS-based methods that address the varying conditions including the understanding of slums in different contexts are yet not available.

\subsection{Research objectives}

The main objective of this study is to develop and test remote sensing based methods for characterising and identifying slum areas using VHR satellite imageries. To achieve this objective, the following specific objectives have been defined.

1. The first objective is to develop an ontological framework to conceptualize slums using morphological indicators of the built environment.

2. The second objective is to determine the transferability of an OOA method to identify and classify slums using a VHR satellite image.

3. The third objective is to develop a hierarchical classification method in an OOA environment to detect and classify slums from VHR image. 
4. The fourth objective is to quantify the uncertainties related to image interpretations of urban slums.

\subsection{Outline of the thesis}

This thesis consists of six chapters. The four core chapters (2-5) correspond to the research findings related to each of the aforementioned objectives respectively.

Chapter 1 presents a brief research background, rational for the selection of the research topic, research objectives and outline of the thesis.

Chapter 2 addresses the first objective of the thesis. Taking UN-HABITAT's definition of slum household as a basis, this study proposes an ontological framework that conceptualizes slums using morphological indicators of the built environment. This framework, referred as generic slum ontology (GSO), integrates expert knowledge and is to be used as a basis for image based slum identification. It focuses particularly on physical aspects that may be derivable through remote sensing, i.e. identifying which indicators could be used to distinguish slums from non-slum areas.

Chapter 3 deals with the second objective of this thesis. It demonstrates a case of applicability of GSO and explores the transferability of an OOA method for slum classification developed on a single image area to other image areas. To include contextual knowledge, expert knowledge is encoded in the ontological framework to classify slums in the three subsets of GeoEye image of Ahmedabad city, India. A set of image-based parameters are identified as most stable or transferable for differentiating slums from non-slum areas in all the subsets.

Chapter 4 addresses the third objective that proposes a hierarchical method for slum detection by using a combination of texture and spatial metrics. The method is applied to a Quickbird image of the city of Pune, India. Integration of expert knowledge of slums is done in the form of a local slum ontology which also demonstrates the applicability of the GSO on a different context.

Chapter 5 presents the research related to the fourth objective. It demonstrates a method to quantify the uncertainties of slum interpretations performed by experts. Delineations done in three different contexts are considered and factors responsible for uncertainties in distinguishing slums from non-slum areas are studied. The study cases are Nairobi, Cape Town and Ahmedabad. 
Chapter 6 presents the main research findings and conclusions corresponding to each research objective. The main contributions and novelty of scientific output of this thesis are discussed followed by recommendations for future research to further improve slum identification and classification using remote sensing. 


\section{Chapter 2}

\section{An ontology of slums for image-based classification*}

"I speak to maps. And sometimes they say something back to me. This is not as strange as it sounds, nor is it an unheard of thing. Before maps the world was limitless. It was maps that gave it shape and made it seem like territory. like something that cauld be possessed, not just laid waste and plundered. Maps made places on the edges of the imagination seem graspable"

- Abdubrazak Gurnah

* This chapter is based on the article:

Kohli, D., Sliuzas, R., Kerle, N., Stein, A., 2012. An ontology of slums for image-based classification. Computers, Environment and Urban Systems 36, 154-163. 


\begin{abstract}
Information about rapidly changing slum areas may support the development of appropriate interventions by concerned authorities. Often, however, traditional data collection methods lack information on the spatial distribution of slum-dwellers. Remote sensing based methods could be used for a rapid inventory of the location and physical composition of slums. (Semi-) automatic detection of slums in image data is challenging, owing to the high variability in appearance and definitions across different contexts. This paper develops an ontological framework to conceptualize slums using input from fifty domainexperts covering sixteen different countries. This Generic Slum Ontology (GSO) comprises concepts identified at three levels that refer to the morphology of the built environment: the environs level, the settlement level and the object level. It serves as a comprehensive basis for image-based classification of slums, in particular, using object-oriented image analysis (OOA) techniques. This is demonstrated by with an example of local adaptation of GSO and OOA parameterization for a study area in Kisumu, Kenya. At the object level, building and road characteristics are major components of the ontology. At the settlement level, texture measures can be potentially used to represent the contrast between planned and unplanned settlements. At the environs level, factors which extend beyond the site itself are important indicators, e.g. hazards due to floods plains and marshy conditions. The GSO provides a comprehensive framework that includes all potentially relevant indicators that can be used for image-based slum identification. These characteristics may be different for other study areas, but show the applicability of the developed framework.
\end{abstract}




\subsection{Introduction}

At present about half of the world's population is estimated to live in cities. According to the projection by a recent report of UN-HABITAT (UN-HABITAT 2010), urban areas will be the epicenter of almost all of the world's population growth in the next 30 years. In addition to this growth, planners and governments in developing countries face the challenge of high growth rates of poverty. Whereas poverty was once seen as being a largely rural problem, today we speak of the urbanization of poverty (Kombe 2005). Urban poverty is manifested most conspicuously in the proliferation and expansion of slums, which are home to a growing proportion of urban dwellers. Though there have been collective efforts at global and national levels to improve the condition of slum dwellers, the world's slum population is estimated to reach 889 million by 2020 (UN-HABITAT 2010).

Addressing the problems of slums requires comprehensive information on these highly dynamic areas as a basis for coordinated actions at local, national and international level. As slums are directly related to poverty, consistent information on the nature and extent of slums is useful for planning, implementing and monitoring urban poverty alleviation programs. The systematic quantification of slums requires methods to identify and define them spatially in a consistent manner in support of geographical targeting in slum intervention programmes (Sliuzas et al. 2008a). Further, considering the rapid change in the structures of such settlements as a result of expansion, infill, relocation and extension, automatic techniques could help in slum monitoring. For these reasons, there is a growing interest in developing and exploiting robust approaches for collecting information on slums. These approaches include participatory methods (Lemma et al. 2006, Joshi et al. 2002, Hasan 2006), mapping census data with GIS (Baud et al. 2009, Baud et al. 2008) as well as very high resolution (VHR) remote sensing based methods for mapping slums (Mason and Fraser 1998, Sliuzas et al. 2008b). In particular RS methods could provide updates also for the parts of the world where census data or other data are not readily available (Weeks et al. 2007).

Traditionally, urban slum and non-slum settlements have been differentiated based on administrative definitions or income-based indicators. The official data collection methods in most developing countries are typically head-count based (census) that do not provide detailed spatial information about the concentration or location of slum-dwellers. Furthermore, there can be contradictions between the definition of slums in different countries that often develop their own definitions, making data exchange, comparisons and monitoring of intervention plans difficult. 
In this study, we first provide a brief review of the three major approaches of slum mapping, including the use of very high resolution (VHR) optical images. Recent research in the use of VHR images has been moving from pixel-based methods to object-oriented image analysis approaches, which requires a clear conceptualization of the object of interest. Such a conceptualization forms the basis of image-based mapping, but also needs to address the huge variability in slum characteristics and appearance across different contexts. The purpose is to develop contextual OOA methods that closely resemble the cognitive approach of visual image analysis for slum identification and mapping. We follow an ontological approach to conceptualize slums using indicators related to the morphology of the built environment. The ontological framework provides a comprehensive description of spatial characteristics and their relationships to represent and characterize slums in an image.

\subsection{Ontology}

The first use of the term "Ontology" is attributed to Aristotle around 340 BC as representing reality by breaking it down into concepts, relations and rules (Agarwal 2005). Gruber (1993) defined ontology as a specification of conceptualization, i.e. an abstract and simplified representation of real-world entities. According to Benslimane et al. (2000), "Ontology provides a formal representation for the concepts of an application and relationships among them, thus capturing the intended meaning of the terms of the domain of interest". Ontology thus provides a basic knowledge skeleton around which a knowledge base can be built (Swartout and Tate 1999). For Chandrasekharan et al. (1999), ontology acts as the representation vocabulary for a specific domain, defining the terms that are used. These definitions show slight variations in the terminology to define ontology, depending on the viewpoint of the author (Winter 2001). We follow the definition of Sowa (2000), who considers the ontology of a specific domain to consist of the domain vocabulary, all its essential concepts, their classification, taxonomy, relations and domain axioms.

Ontologies have acquired considerable importance in knowledge representation in recent years. They are used to represent knowledge in biomedical science (Matei 2008)and computer science (Guarino 1995, Guarino 1997). Ontologies have been applied in landscape-studies (Lepczyk et al. 2008), in crisis management (Fan and Zlatanova 2010, Li et al. 2008), in GIScience for sharing geographic information in a standardized way (Agarwal 2005) and in urban studies to facilitate communication between information systems, stakeholders and urban experts (Belmonte et al. 2008, Fonseca et al. 2000, Teller et al. 2007). The common idea behind all these studies has been to develop a common understanding of the domain of interest and facilitate information exchange. Recently, ontologies have been applied in remote 
sensing studies to address uncertainty in object-reconstruction from an image, based on knowledge of the real world (Agouris et al. 1999).

We expand upon the ontological work of Hofmann et al. (2008) to develop a Generic Slum Ontology (GSO). We use the "durable housing" indicator of UNHABITAT (2003b), which defines a slum household as one lacking in any one of five factors: secure tenure, access to safe water, access to sanitation, sufficient living area and durability of housing. Durable housing refers to: permanency of structure - walls, roof and floor; compliance of building codes; well maintained and structurally sound; not be located on a hazardous location (e.g. near toxic waste; on a flood plain; on an unstable slope). VHR images are well suited to quantify the durability of housing, as many of these physical characteristics can be readily observed. In addition, we use knowledge from experts to identify other indicators for slum detection, building upon the study by Ebert et al. (2009), who used a set of image-derived physical proxy variables to assess urban social vulnerability. We use the physical characteristics of the urban environment apparent from VHR images to characterize slums using the generic slum ontology as a basis.

\subsection{Previous work on slum identification and mapping}

In this section we discuss three different slum mapping approaches, based on (i) census data, (ii) participatory methods and (iii) advanced remote sensing image analysis. Throughout this study, the terms informal settlements and slums are used interchangeably, since informal settlements are often referred to as slums and vice-versa in previous literature (Hofmann et al. 2008, UNHABITAT 2003a). Though by definition, not all slums would be considered as informal settlements, e.g. the inner-city slums which are dilapidated structures with formal rights. However, according to UN-HABITAT's definition informal settlements are always considered to be slums by virtue of the lack of tenure security. Moreover, the great majority of informal areas are occupied by relatively poor residents and will usually have at least one additional form of deprivation (i.e. related to water, sanitation, overcrowding or durable housing).

\subsubsection{Census-based approach}

The census-based approach uses data collected through census surveys as a basis of deprivations/poverty mapping. Census data contain details on the inhabitants residing in a country aggregated to small areas to protect individual privacy. They consist of data on social, economic and habitat/infrastructural aspects at the level of an enumeration unit (Baud et al. 2008). Baud et al. (2008) used census data to map concentrations of poverty at the ward level in Delhi, India by developing an index of multiple deprivation. Similar studies 
were conducted in three other Indian cities using a multi-criteria model within GIS for the identification of multiple deprivation hotspots (Baud et al. 2009). Weeks et al. (2007) used census data from Accra, Ghana, to create a slum index based on the UN slum indicators for each Census enumeration area (EA), using it to measure the concentration of slums in each EA. High correlations were found between the slum index, the socio-economic characteristics of neighborhoods and certain land cover metrics derived from VHR satellite imagery (Weeks et al. 2007).

As census data are routinely collected in many countries, they provide an interesting option for poverty/slum mapping. In addition, they mostly provide information at the household level. Their utility is restricted by the long temporal gap (often a decade, in most countries) between two census surveys (Hall et al. 2001, Ebert et al. 2009), and time needed to check raw data and collate and release derived statistics to users (Martínez 2009). Considerable socio-economic and physical changes can occur on the ground, especially in slum areas. Also, the degree of data aggregation can be a problem. For example, census wards in Delhi vary in size between 1500 households to more than 57,000 households.

\subsubsection{Participatory approach}

The participatory approach involves mapping the details of informal settlements at individual or household level with the cooperation of slumdwellers (Joshi et al. 2002). Hasan (2006) used this approach to survey and map infrastructure details of informal settlements in Karachi. Karanja (2010) presented a full enumeration of households and slums in Kisumu, Kenya. Earlier, Lemma et al. (2006) employed rapid appraisal techniques that integrate local knowledge with Geographic Information Technology (GIT) in a participatory approach that involved input from stakeholders and visual image interpretation using satellite images and aerial photographs. As communitybased slum enumeration is also being actively promoted by international slum networks, such as Slum Dwellers International, it will likely become a widely used approach and tool (see http://www.sdinet.org/ritual/enumerations/).

Participatory approaches can help to generate spatial and non-spatial information in the form of thematic GIS layers that provide a supplement to socio-economic surveys and feed into slum-related policy development and planning. From the perspective of slum communities this is especially important, as the process of enumeration generates much needed data that are useful both for the slum communities themselves, as well as in negotiations with government bodies concerning needs and services. However, the detailed level of information provided by the participatory approach is time consuming to collect. In cities with extensive slum settlements, this information may be difficult to upscale to larger areas. Other methods that can reduce enumeration 
resources and can systematically generate city wide slum maps from VHR images are thus required. Such maps could be used as an input for urban policy, growth monitoring and various planning processes.

\subsubsection{Remote sensing based approach}

The remote sensing (RS)-based approach uses image processing techniques to map slums from satellite imagery. RS can provide spatially disaggregated data with high temporal consistency, which can facilitate rapid monitoring and effective intervention of slums by local authorities. Mason and Fraser (1998) demonstrated automated shack extraction from a VHR aerial imagery. Visual interpretation from VHR images has been used to extract concentrations of urban poverty (Sliuzas and Kuffer 2008, Angeles et al. 2009). Baud et al. (2010) used VHR images to identify precise patterns within deprived areas using physical variables such as built-up density, building size and site suitability. Visual interpretation methods, however, require extensive local expert knowledge to produce reliable results. Moreover, automating the interpretations can lead to more objective and faster results. Jain et al. (2005) suggested and later demonstrated (Jain 2007) that studying informal development through image classification could explain the patterns over time and space. Weeks et al. (2007) used Ridd's vegetation, impervious surface, bare soil (V-I-S) model (Ridd 1995) to classify slum neighborhoods in Accra, Ghana. However, pixel-based classification, as used in these studies, is unable to represent the heterogeneity of an urban environment (Myint et al. 2011).

With VHR images, OOA techniques for classification have proven to be accurate in urban applications (Sliuzas et al. 2008b, Ebert et al. 2009). Such a multiscale approach may capture the heterogeneity of urban areas by following a hierarchy for object-classification that includes contextual information for objects and non-physical features (Ebert et al. 2009), specifically for informal settlements (Hofmann 2001). These opportunities are coupled with higher levels of complexity in terms of deriving real-world knowledge through remote sensing. Therefore, it is important to understand and integrate epistemological and ontological aspects of real-world objects for wider OOA applicability (Blaschke 2010). Hofmann et al. (2008) extended earlier work (Hofmann 2001) by introducing the concept of ontology to first define informal settlements as a basis for object-based classification. Their ontological framework was able to represent conceptual knowledge but the indicators and the absolute thresholds for the variables remained data/scene specific. The work described in this study builds on this ontological approach, but instead of focusing on a particular study area, we develop a generic ontological framework for slums as a basis for object-based classification across different contexts. 


\subsection{Ontological framework for slums}

The GSO consists of characteristics at three spatial levels: object level, slum settlement level and the slum environment. We follow this multi-scale approach considering the variable nature of slums across different contexts, by considering a set of indicators at different scales for a slum as a vague phenomenon. The ontology development process is based on the one suggested by Fernández López et al. (1999) named "Methontology", comprising three phases: specification, conceptualization and implementation.

\subsubsection{Specification}

Specification refers to determining the domain, its scope and the set of terms to be represented in an ontology (Fernández López et al. 1999). It creates a clear vision of the ontology's coverage, its intended use and the types of questions the information in the ontology should provide answers to. On the basis of image-based identification and classification of slums, we focus on those characteristics that are observable from VHR images. To collate information on the main concepts and relationships associated with slums, first a literature review was carried out to form an initial list of potential indicators. To refine this list and to include any missing indicators, we carried out semistructured surveys and interviews with fifty domain experts (urban planners, remote sensing experts, academics and local administrators) with knowledge in remote sensing and field experience of slums. The experts were from Asia, Sub-Saharan Africa (SSA), Europe, Latin America, North America and Australia. They were asked to fill the questionnaire considering the slums they were most familiar with. All experts referred only to slums from countries in Asia, SSA or Latin America. Table 2-1 shows the names and numbers of the countries in Asia, SSA or Latin America mentioned by respondents. As is evident from the table, the majority of responses referred to SSA and Asia. The low representation of Latin America and the absence of several other regions where slums are known to exist is a limitation of the study. 
Table 2-1 List of countries referred to by respondents in the expert survey grouped by region.

\begin{tabular}{lll}
\hline Region & Countries & $\begin{array}{l}\text { Number of } \\
\text { responses }\end{array}$ \\
\hline Asia & $\begin{array}{l}\text { Bangladesh, India, Indonesia, Nepal, } \\
\text { Philippines } \\
\text { Sub-Saharan Africa, } \\
\text { Rhana, Namibia, Malawi,Mozambique, } \\
\text { Rwanda, South Africa, Tanzania, Uganda } \\
\text { Argentina, Venezuela, Jamaica }\end{array}$ & 15 \\
\hline
\end{tabular}

\subsubsection{Knowledge acquisition}

Methontology identifies knowledge acquisition as an important and independent step for ontology development (Fernández López et al. 1999). We used literature review, interviews and semi-structured survey for knowledge acquisition. The survey consisted of questions on how domain experts defined slums (open-ended question), specific characteristics of slums in their respective context (Table 2-2) and a section for delineating slums (Figure 21). Their responses were used to understand how experts perceive a slum. They also helped to comprehend the decision-making steps employed by experts to identify slums in an image. The responses provided insight into the variability and similarities of slums in various contexts. Based on this, we finalized the list of indicators to form the generic slum ontology as a comprehensive set to capture main slum features.

To capture the basic understanding of slums in different contexts, we analysed the definitions provided by the experts. Content analysis was used to categorize answers from respondents by studying all responses to develop a set of general deprivation issues that frequently occurred in their responses (Table 2-3). Subsequently, we counted the number of responses that referred to each issue. The issue basic services include those responses that mentioned any one or a combination of: access to water, sanitation, drainage and electricity. The issue socio-economic conditions include responses referring to low income, poor/sub-standard living conditions, schools and health facilities. Finally, the responses related to the physical conditions were allocated in the physical infrastructure issue including responses related to the physical condition of houses, absence of or irregular roads, high building density, hazardous location, poor environmental condition, durability (remote sensing derivable). The category others refer to responses mentioning tenure or overcrowding in their definitions. As is evident from the table, 86 percent of the respondents referred to physical infrastructure while defining slums. This substantiates the potential of using remote sensing for slum identification and mapping. 
Table 2-2 Characteristics of slums for each general indicator that were specified by domain experts.

\begin{tabular}{ll}
\hline General indicators & Specific characteristics \\
\hline Building characteristics & $\begin{array}{l}\text { Building types, number of floors, footprint in } \mathrm{m}^{2} \\
\text {,building height, roof, wall and floor materials }\end{array}$ \\
Access network & $\begin{array}{l}\text { Type of roads, percentage paved, width, layout } \\
\text { Density }\end{array}$ \\
Settlement shape & $\begin{array}{l}\text { Segetation of roof coverage, open spaces and } \\
\text { Location }\end{array}$ \\
Neighborhood characteristics & Site condition of slum \\
\hline
\end{tabular}

We also asked the domain experts to identify and delineate slums in satellite images of four cities acquired from Google Earth (Figure 2-1). Additionally, the experts were asked to list the factors they used for identification and delineation. Finally, they were also asked to mention the reasons, if any, for not being able to identify a slum.

The results of the survey show that experts use eleven basic slum indicators to delineate slums from imagery: building size, roof material, absence of roads, irregular roads, lack of vegetation and open spaces, density (compactness), irregular shape of settlement, association with neighbouring areas, texture and locality. The combination of indicators, however, varied from place to place. This information was used in conceptualizing the basic knowledge about slums and finalizing the set of indicators.

Table 2-3 Number and percentage of experts who referred to a general deprivation issue while defining slums.

\begin{tabular}{lcccc}
\hline \multirow{2}{*}{ Responses } & \multicolumn{4}{c}{ General Deprivation Issues } \\
\cline { 2 - 5 } & $\begin{array}{c}\text { Basic } \\
\text { services }\end{array}$ & $\begin{array}{c}\text { Socio- } \\
\text { economic } \\
\text { conditions }\end{array}$ & $\begin{array}{c}\text { Physical } \\
\text { infrastructure }\end{array}$ & $\begin{array}{c}\text { Others (tenure, } \\
\text { overcrowding) }\end{array}$ \\
\hline $\begin{array}{l}\text { Number of } \\
\text { respondents }\end{array}$ & 35 & 30 & 43 & 38 \\
Percentage & 70 & 60 & 86 & 76 \\
\hline
\end{tabular}



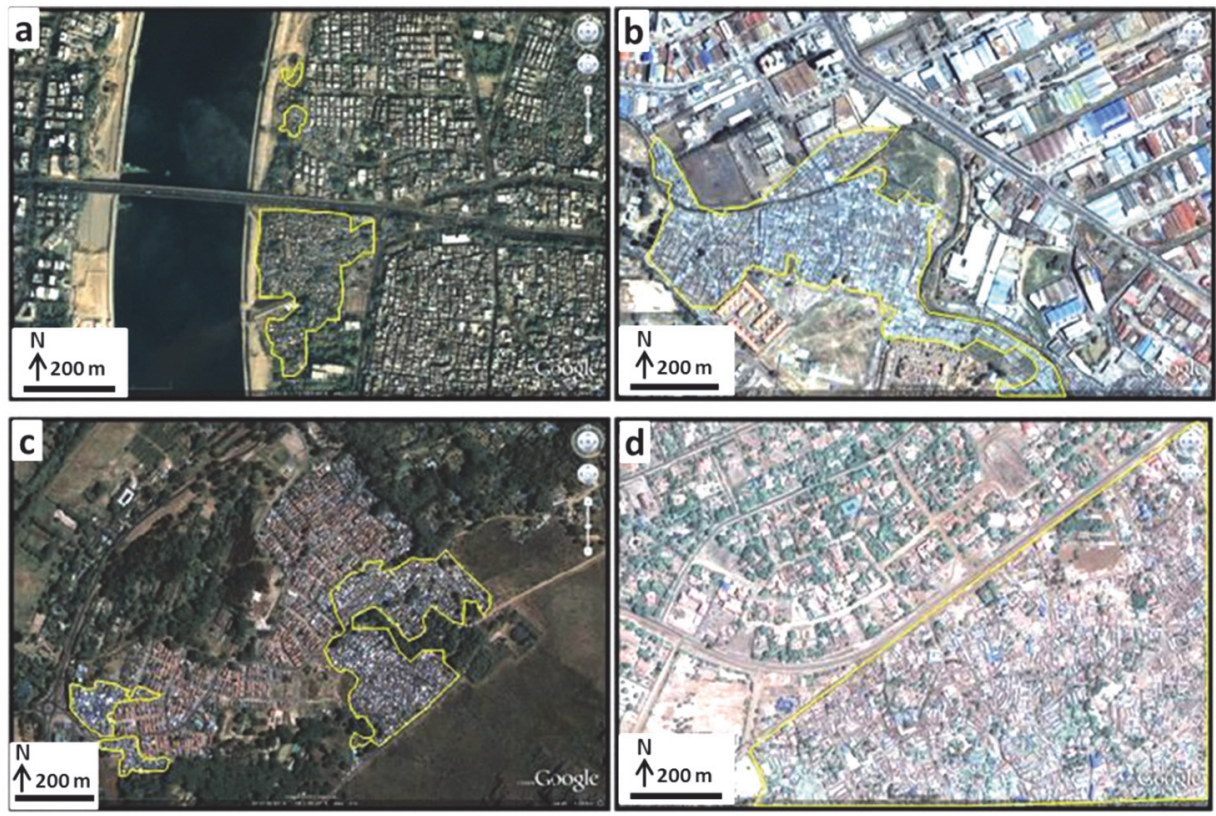

Figure 2-1 An example of slum delineation in different contexts by an Indian expert. City names: a) Ahmedabad (India), b) Nairobi (Kenya), c) Cape Town (South Africa), d)Kisumu (Kenya). Source: Google Earth.

\subsubsection{Conceptualization}

Conceptualization refers to organizing and structuring the knowledge acquired during the specification phase in a semi-formal way, using a set of immediate representations that both the domain experts and ontologists can understand (Fernández López et al. 1999). Figure 2-2 shows the classification (taxonomy) starting from object level to a higher level, referred to as environs (Gasevic et al. 2009). It shows the concepts at three spatial levels, their hierarchy and the indicators associated with each level. Axioms, such as close to, has, is in, is of, are used to specify relation between indicators and their attributes. The description of concepts is as follows:

- Environs

- Settlement level

- Object level
Surroundings of the settlement, i.e. its location with respect to neighboring land uses, or areas with specific environmental conditions, such as hazardous areas.

Overall form/shape/density of the settlement

Components of the settlement, such as characteristics of buildings and roads. 


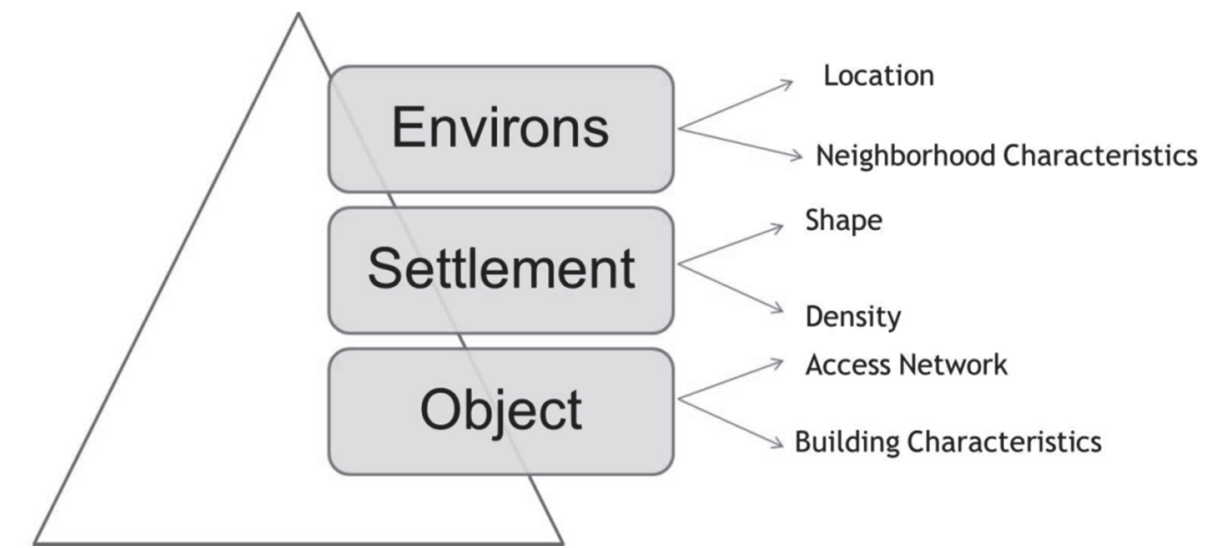

Figure 2-2 The six general indicators categorized to form a hierarchy to represent concepts at three spatial levels.

\subsubsection{Building characteristics}

A building is a structure with roof and walls. Buildings in a slum settlement tend to have characteristics that are different from those in non-slum areas. Some authors have explicitly described features of buildings while discussing slums in different contexts (Neuwirth 2005, see Davis 2006). 76 percent of the domain experts referred to building characteristics while defining slums. All attributes related to a building are represented in Figure 2-3; however, not all of them are detectable from satellite images. A combination of attributes such as roof type, footprint, shape and orientation can be used to identify slum buildings using remote sensing. There may be regional similarities in slum characteristics. All the experts referring to SSA mentioned the roof material to be either tin or iron sheets in addition to others. The materials from Asian context include mud, plastic or straw thatch etc. with color being a distinguishing feature (Figure 2-1). 63 percent of the responses from the SSA context said that the type of building is detached, whereas 73 percent from Asian context mentioned the type as attached. Generally, the footprints of dwellings in a slum are smaller than in planned settlements, and their shape and orientation do not generally comply with planning standards. 


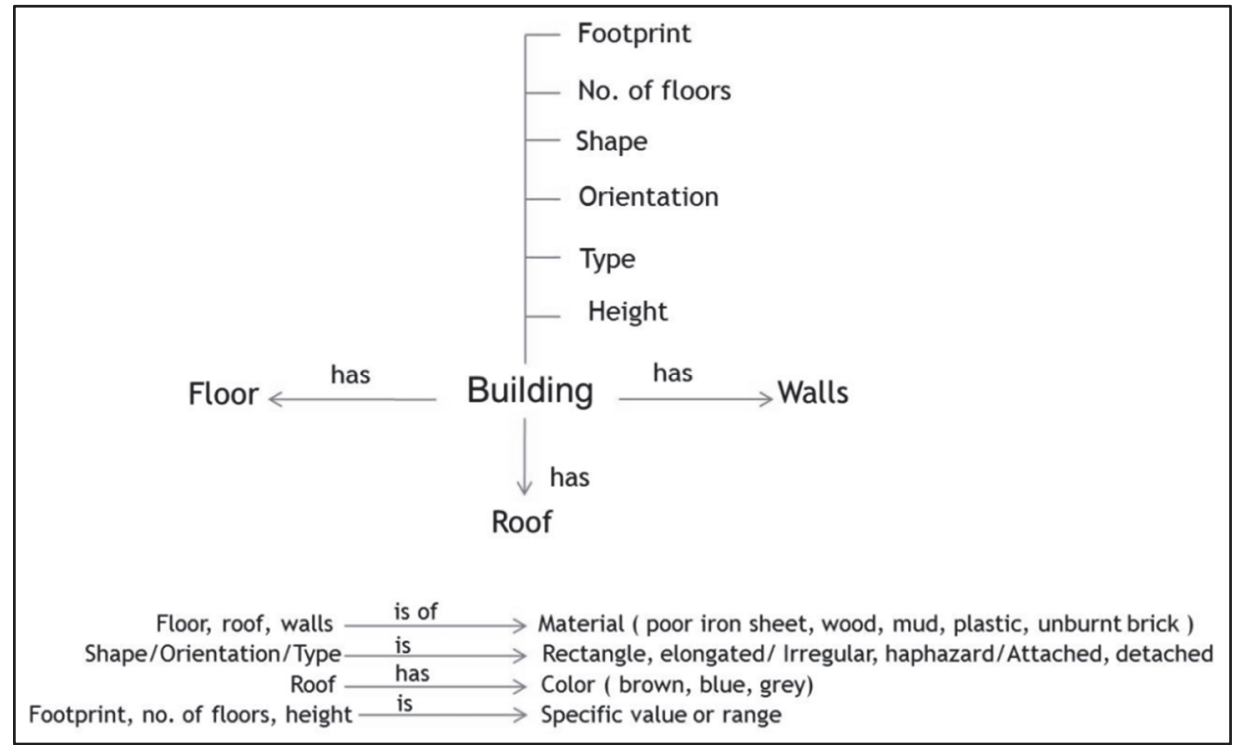

Figure 2-3 Diagram of building attributes with the corresponding values derived from the expert survey.

\subsubsection{Access network}

A slum settlement generally has an irregular access network with variable street types, surfaces and widths (Figure 2-1). The Oxford dictionary (OED 2011) defines a street as "a public road in a city, town, or village, typically with houses and buildings on one or both sides". Figure2-4 gives an overview of attributes related to street layout and connectivity. According to our field experience and previous literature (Nobrega et al. 2008, Sliuzas et al. 2008b, Hofmann et al. 2008), road characteristics are an important indicator for slum identification, as most slums lack proper road networks and existing roads are often poorly connected to surrounding areas. The expert survey confirms this. Terms referring to access/roads appeared in 50 percent of the respondent's definitions of slums, and 96 percent of the respondents said that the road layout in a slum is irregular, while only 14 percent said that it is likely to be well connected with neighboring areas. Slums may also only have footpaths or access streets that are un-paved.

\subsubsection{Shape}

Slums tend to follow the shape of features such as roads, railways or drainage channels, due to comparatively easy availability of public land (Neuwirth 2005, Davis 2006). Slums may also be adjacent to planned areas or major ring roads. The terms elongated or linear were used by 59 percent of the experts to specify the shape of slum in their respective context, while 50 percent also mentioned irregular. This indicator can be used to find probable location of slums based on distance from such features. 


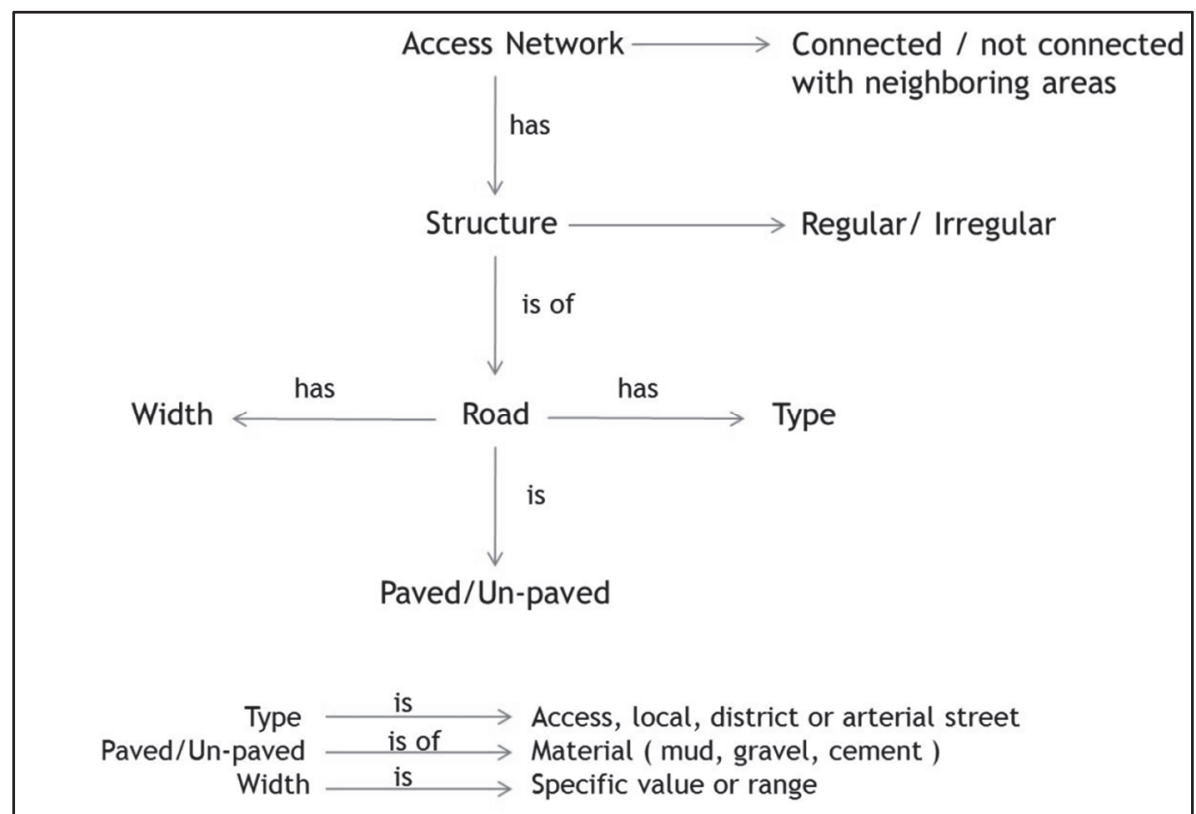

Figure 2-4 Diagram of access network characteristics observable via VHR images with the corresponding values derived from the expert survey.

\subsubsection{Building density}

Building density is a key variable for identifying slums. Slums generally have high roof coverage with no open spaces or vegetation (Neuwirth 2005). For the Asian context, 14 (93 percent) out of 15 of the experts specified roof coverage as 80 percent or above. By comparison, in the SSA context only 12 (60 percent) out of 20 experts who responded to this question specified such high densities. Thus, density differs locally, and it also depends upon the age of the settlement. The association with surrounding areas (planned) plays an important role in using this indicator for identifying slums. Building density plays a central role in a typical slum definition but both dense formal neighborhoods, and low density slums are sufficiently common worldwide to ensure ambiguity. Therefore, density cannot be used as the sole variable for slum identification.

\subsubsection{Location}

Slums frequently develop on hazardous sites where other development is not possible. A site is defined as a plot or area of land that has been or is intended to be constructed or used for some purpose. Table 2-4 shows the number and respective percentages of respondents who said that a slum is likely to have a specific site condition. Slums may be found, for example, in flood zones, marshy areas, along railways and on steep slopes (Figure 2-5) (Davis 2006). In Latin American cities, for example, slums may be located on high and 
unstable slopes, whereas in India the majority of slums are located next to linear features such as roads and canals. These indicators are context dependent, as also became evident from the expert survey. However, the percentage of responses for proximity to railway lines and roads is relatively high in both Asia and SSA. By proximity or closeness we mean the spatial distance that could have a detrimental effect on the living conditions of the people living in a slum. The expression is context-dependent and the implications of distance will vary from place to place. RS can be used to locate probable slum settlements based on hazard zones, height information and GIS layers of the area.

Table 2-4 Number and percentage of experts identifying a site or neighborhood condition as being a likely characteristic of an Asian or SSA slum.

\begin{tabular}{|c|c|c|c|c|}
\hline \multirow{2}{*}{$\begin{array}{l}\text { Site or neighborhood } \\
\text { condition }\end{array}$} & \multicolumn{2}{|c|}{$\begin{array}{l}\text { Asia } \\
\text { (15 valid responses) }\end{array}$} & \multicolumn{2}{|c|}{$\begin{array}{l}\text { SSA } \\
\text { (29 valid responses) }\end{array}$} \\
\hline & Number & Percentage & Number & Percentage \\
\hline Flood zone & 6 & 40 & 14 & 48 \\
\hline Steep slopes & 3 & 20 & 10 & 34 \\
\hline $\begin{array}{l}\text { Close to railway lines, } \\
\text { highways, major roads }\end{array}$ & 13 & 87 & 22 & 76 \\
\hline $\begin{array}{l}\text { Close to high-voltage power } \\
\text { lines }\end{array}$ & 5 & 33 & 17 & 59 \\
\hline Close to hazardous industries & 8 & 53 & 9 & 31 \\
\hline $\begin{array}{l}\text { Connected to infrastructure in } \\
\text { neighboring areas }\end{array}$ & 4 & 27 & 9 & 31 \\
\hline $\begin{array}{l}\text { Close to/inside neighborhood } \\
\text { of low socio-economic status }\end{array}$ & 11 & 73 & 16 & 55 \\
\hline $\begin{array}{l}\text { Close to / inside neighborhood } \\
\text { of middle/high socio-economic } \\
\text { status }\end{array}$ & 4 & 27 & 19 & 66 \\
\hline Close to employment & 14 & 93 & 21 & 72 \\
\hline
\end{tabular}




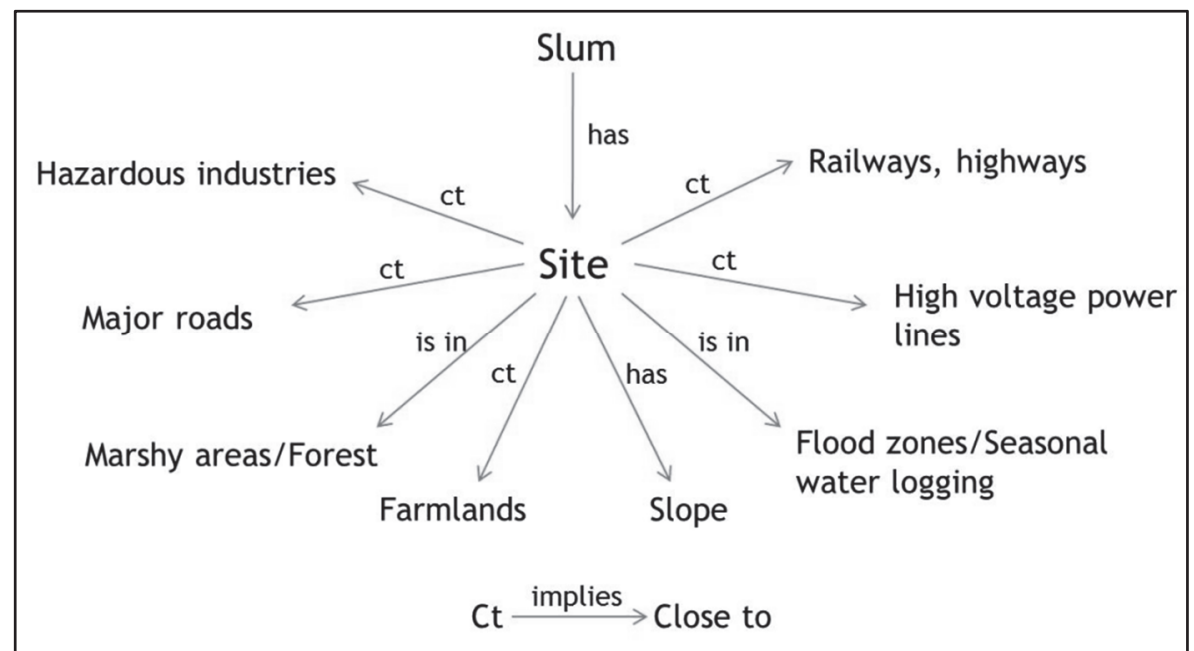

Figure 2-5 Diagram showing possible site conditions of a slum derived from expert survey.

\subsubsection{Neighborhood characteristics}

The location of a slum settlement depends on socio-economic factors (Figure 2-6). Slums are often formed close to opportunities for unskilled or low skilled jobs. The opportunities can be in higher socio-economic status neighborhoods, which lead to the development of informal houses in their vicinity. Industries and central business districts (CBD), in most developing countries, also attract slum development due to employment possibilities such locations offer (Neuwirth 2005, Davis 2006). This is evident from the expert survey also. 93 percent of respondents from the Asian context and 72 percent of the experts referring to SSA said that it is likely that a slum is located close to sites of employment opportunities. Slums further tend to develop in existing low socioeconomic areas due to affordable living expenses (Table 2-4).

\subsubsection{Implementation- local adaptation of GSO}

Implementation involves the transformation of conceptual model into an implemented model (Fernández López et al. 1999). To illustrate this, we provide one example of local adaptation of GSO to one of the cities used in the expert survey. We emphasize the need for local adaptation of the concepts mentioned in the generic ontology, as not all indicators would apply to all areas. 


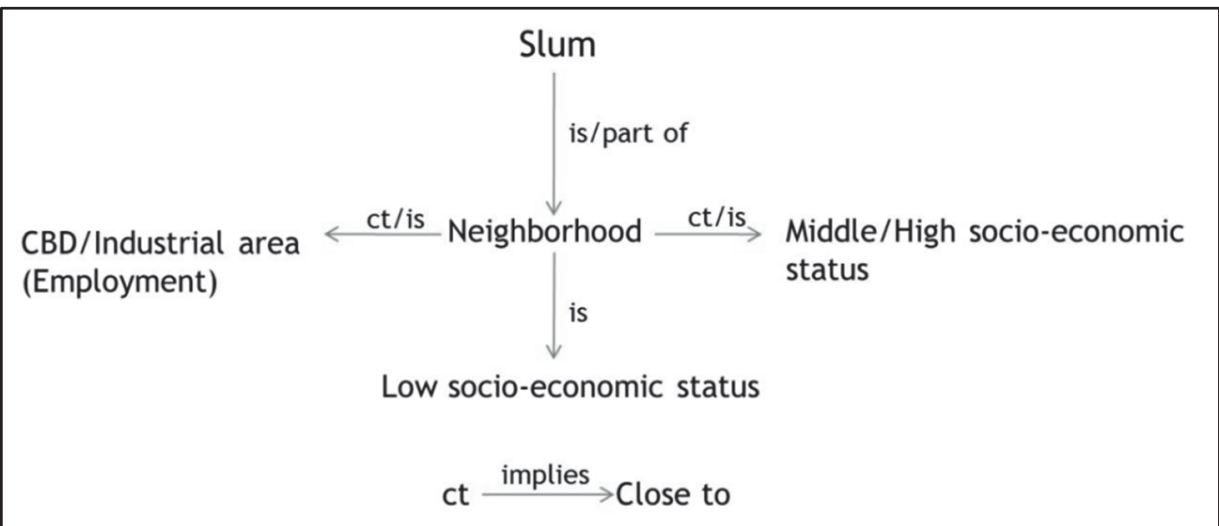

Figure 2-6 Diagram of socio-economic factors that are possible driving forces for development of slums according to domain expert survey.

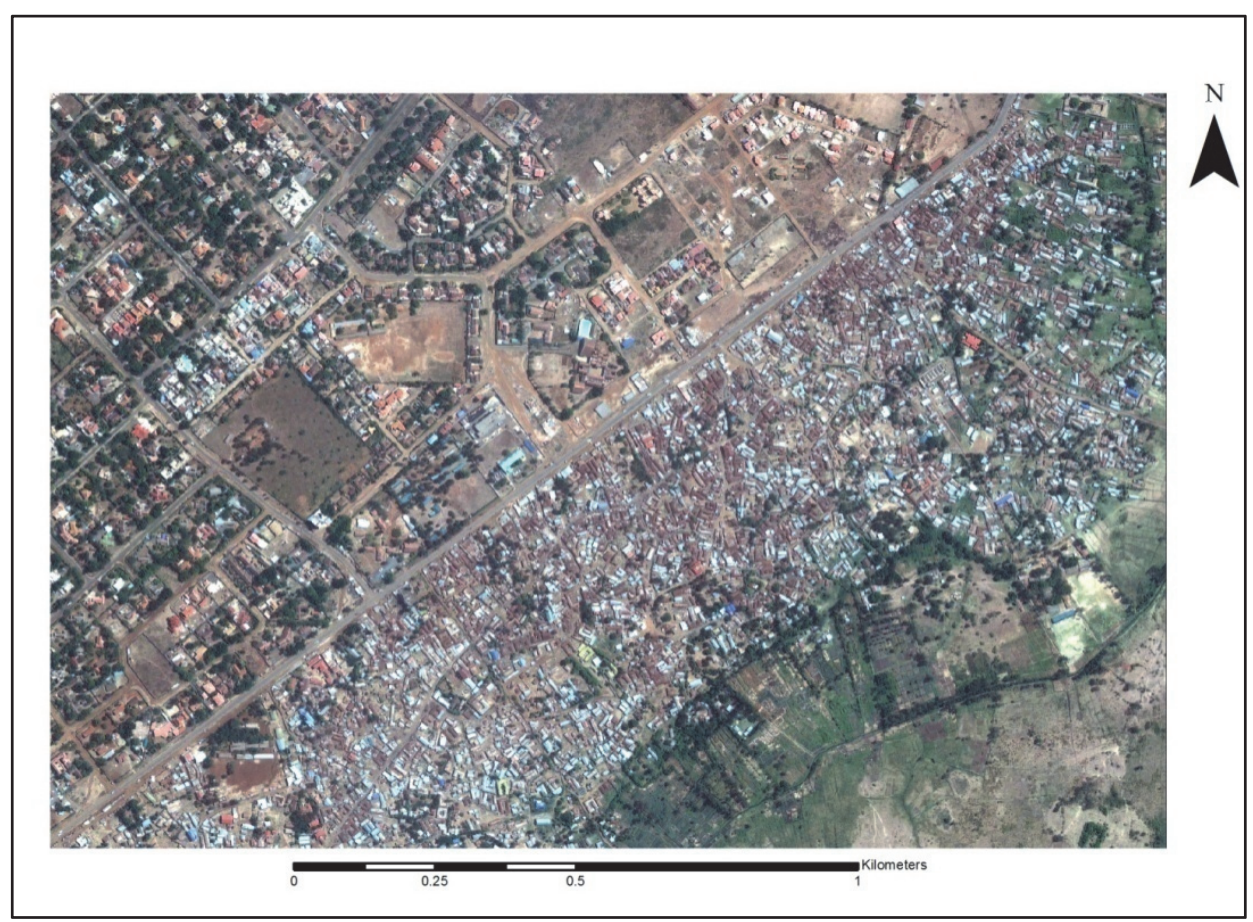

Figure 2-7 A snapshot of Kisumu, Kenya. The Nyalenda slum area is clearly visible below the major road running diagonally from bottom left to top right.

Kisumu (Figure 2-7) is the third largest city in Kenya, with a population in 2004 estimated at 500,000. It is one of the poorest cities in Kenya with more than 60 percent of population living in slums. Urbanization and lack of planning over the past decades have led to uncontrolled slum formation, with slum settlements encircling the entire inner planned area of the city. This is evident from the clear contrast between the planned areas and the slums in the image 
(Figure 2-7). We used a subset of Kisumu for slum delineations by respondents in the expert survey. The experts were provided snapshots of Google Earth images in $\mathrm{kml}$ format that could be reopened with the same extents (Figure 21). Figure 2-8 shows an example of slum outlines by 5 experts for Kisumu. The slum boundaries have deviations as 3 respondents in this example excluded patches of open areas. 1 respondent included one open area while the remaining expert (green colored) outlined the area including both open areas. The difference in boundaries could also be due to shift in the image extent while digitizing in Google Earth. Nonetheless, there is considerable overlap between the delineations and the indicators used in the decision-making process. The following indicators recurred while reading through the respondent's factors for slum delineations in this context: Color, shape, size and orientation of buildings, chaotic street pattern, irregular layout, high density, less vegetation, contrast with planned area and haphazard development.

Table 2-5 summarizes the observations of visual image interpretation integrated with the knowledge of slums from local experts (UN-HABITAT 2005). These observations helped to understand the characteristics of slums in Kisumu. Four main indicators comprising spectral range, geometry, texture and association were chosen to translate the slum knowledge into specific OOA parameters.

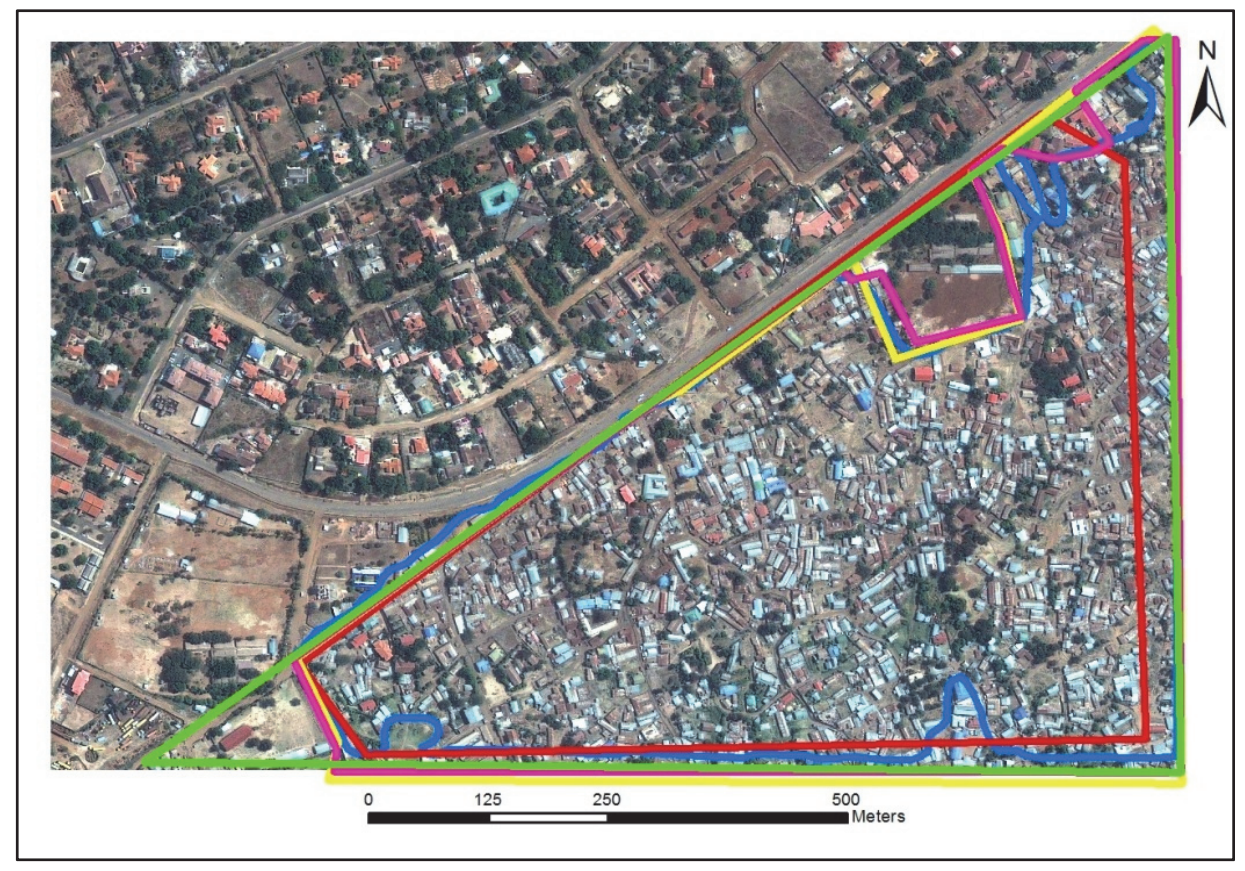

Figure 2-8 Delineations of slums from a snapshot of Kisumu, Kenya produced by 5 randomly selected domain experts. 
Table 2-5 Translation of visual observation and expert knowledge to image-based indicators.

\begin{tabular}{lll}
\hline Image observation & Knowledge of slums from local experts & Indicators \\
\hline $\begin{array}{l}\text { Buildings range from bright grey } \\
\text { to a dark brown color; a few are } \\
\text { bright blue and red. }\end{array}$ & $\begin{array}{l}\text { Majority of roofs constructed from } \\
\text { galvanized iron sheets, tin, few painted with } \\
\text { blue and red color }\end{array}$ & Spectral \\
Same building with varying colors & $\begin{array}{l}\text { Single building made up of mixture of old } \\
\text { and new iron sheets }\end{array}$ & Shape/ \\
$\begin{array}{l}\text { Rectangular and elongated shape } \\
\text { of buildings, varying building size }\end{array}$ & $\begin{array}{l}\text { Row houses for rental purpose - many units } \\
\text { in a single building }\end{array}$ & Geometry \\
Irregular orientation of buildings & $\begin{array}{l}\text { Buildings built haphazardly due to lack of } \\
\text { planning }\end{array}$ & \\
$\begin{array}{ll}\text { Unpaved and irregular roads } \\
\begin{array}{l}\text { Irregular shape of slum } \\
\text { settlements }\end{array}\end{array}$ & $\begin{array}{l}\text { Slums form a belt around the planned area } \\
\text { and are along the major roads. }\end{array}$ & \\
$\begin{array}{l}\text { Settlements surrounded by } \\
\text { farmlands }\end{array}$ & $\begin{array}{l}\text { Traditional houses/villages being engulfed } \\
\text { by uncontrolled slum development. }\end{array}$ & Association \\
$\begin{array}{l}\text { Settlements in swamps, black soil } \\
\text { Clear shadow in the west of the }\end{array}$ & $\begin{array}{l}\text { Some slums located in marshy and flood - } \\
\text { prone areas }\end{array}$ & Single story buildings mostly \\
Variable densities & $\begin{array}{l}\text { Density of buildings higher than planned } \\
\text { areas }\end{array}$ & Texture \\
\hline
\end{tabular}

Table 2-6 shows the analysis of physical characteristics adopted from the generic ontology that can be used as the basis for identification and classification of slums in this particular context. In Kisumu, the haphazard orientation of individual buildings, irregular and unpaved roads, high density of settlements, and location in flood-prone and marshy areas were the most important observations for slum identification by experts with local knowledge and visual interpretation. These observations were further categorized and analysed based on the ontological framework by assigning potential parameters for each relevant attribute. It is to be noted that a set of relevant attributes or their thresholds will differ for different contexts. Nonetheless, the GSO provides the basic set of indicators relevant to represent slums in an image. 
Table 2-6 Adaptation of ontological concepts from generic slum ontology to the case study and its respective OOA parameterization.

\begin{tabular}{|c|c|c|c|c|}
\hline Level & Indicator & $\begin{array}{l}\text { Interpretat- } \\
\text { ion element }\end{array}$ & $\begin{array}{l}\text { Observation } \\
\text { (Kisumu) }\end{array}$ & $\begin{array}{l}\text { OOA } \\
\text { parameterization }\end{array}$ \\
\hline \multirow[t]{2}{*}{ Environs } & Location & $\begin{array}{l}\text { Slope, pattern, } \\
\text { secondary } \\
\text { data }\end{array}$ & $\begin{array}{l}\text { On flood zones, } \\
\text { marshy areas, close to } \\
\text { farmlands, and along } \\
\text { highways }\end{array}$ & $\begin{array}{l}\text { Association - distance } \\
\text { to features }\end{array}$ \\
\hline & $\begin{array}{l}\text { Neighbor- } \\
\text { hood } \\
\text { characteri- } \\
\text { stics }\end{array}$ & $\begin{array}{l}\text { Pattern, } \\
\text { secondary } \\
\text { data }\end{array}$ & $\begin{array}{l}\text { Surrounding the } \\
\text { planned areas, close } \\
\text { to sites of employment } \\
\text { opportunities }\end{array}$ & $\begin{array}{l}\text { Association - distance } \\
\text { to planned settlement }\end{array}$ \\
\hline \multirow[b]{2}{*}{$\begin{array}{l}\text { Settlement } \\
\text { level }\end{array}$} & Shape & Pattern & $\begin{array}{l}\text { Encircling the major } \\
\text { ring road }\end{array}$ & Geometry - buffer \\
\hline & Density & Texture & $\begin{array}{l}\text { Denser compared to } \\
\text { planned } \\
\text { Low vegetation and } \\
\text { open spaces }\end{array}$ & $\begin{array}{l}\text { Texture - entropy, } \\
\text { contrast, variance, } \\
\text { mean } \\
\text { Geometry - area of } \\
\text { vegetation, open space }\end{array}$ \\
\hline \multirow{8}{*}{$\begin{array}{l}\text { Object } \\
\text { level }\end{array}$} & & Shape & Rectangular & $\begin{array}{l}\text { Geometry - rectangular } \\
\text { /elliptical fit }\end{array}$ \\
\hline & Building & Size & $\begin{array}{l}\text { Range of values - } 10 \\
\text { to } 40 \mathrm{~m}^{2}\end{array}$ & Geometry - area \\
\hline & & Material & $\begin{array}{l}\text { Roofs-corrugated iron } \\
\text { sheets, tin }\end{array}$ & $\begin{array}{l}\text { Spectral - layer mean } \\
\text { values }\end{array}$ \\
\hline & & Color & $\begin{array}{l}\text { Range-grey, brown, } \\
\text { red and blue }\end{array}$ & $\begin{array}{l}\text { Spectral -maximum } \\
\text { difference, brightness }\end{array}$ \\
\hline & \multirow{4}{*}{$\begin{array}{l}\text { Access } \\
\text { network }\end{array}$} & Orientation & $\begin{array}{l}\text { Haphazard } \\
\text { arrangement }\end{array}$ & $\begin{array}{l}\text { Geometry - angle of } \\
\text { buildings and shadows, } \\
\text { main direction }\end{array}$ \\
\hline & & Shape & Irregular & $\begin{array}{l}\text { Geometry - number of } \\
\text { nodes, length }\end{array}$ \\
\hline & & Type & Unpaved access paths & $\begin{array}{l}\text { Spectral - layer mean } \\
\text { value }\end{array}$ \\
\hline & & Width & Range -1 to $3 \mathrm{~m}$ & $\begin{array}{l}\text { Geometry - length } \\
\text { /width ratio }\end{array}$ \\
\hline
\end{tabular}

The detailed analysis of translation of ontological concepts from object to environs level is shown in table 2-6. At object level, the layer mean values and brightness can be used as the main spectral features to represent roof color and its material. Main direction, angle of buildings and shadows are important parameters to classify slums, considering the haphazard nature of buildings in Kisumu slums. The shape features chosen to characterise buildings include rectangular fit, elliptic fit and area. Number of nodes is an important parameter for roads, as irregular roads tend to have higher number of nodes compared to planned roads. Length and width can also be used to classify roads as there is a high concentration of access paths within the slum areas unlike planned settlements, where there are regulations for the length, width and area of roads. Unpaved roads can be classified using spectral signatures. The belt-like shape of slums in Kisumu, which border the planned areas, can be 
characterized by using a buffer around the major road. At settlement level, texture measures can be potentially used to represent the contrast between planned and unplanned settlements. In addition, areas of vegetation and open spaces are relatively rare in slums due to the high density of buildings. At the environs level, most of the slums in Kisumu qualify for hazardous location, i.e. floods plains and marshy land. The slums have typically developed on former farmlands, resulting in dense, haphazard settlements. The context-related features, in terms of association to certain land use and additional GIS layers, can be used to classify slums in this respect. Further, detailed analysis of all the indicators can help to study intra and inter slum variability, e.g. based on different roof colors, densities, presence/absence of roads, development stage (Sliuzas 2004).

\subsection{Discussion}

The absence of a unique and consistent definition of a slum hinders the development of objective, universally applicable slum detection methods using remote sensing and OOA, which require a clear conceptualization of the object of interest. Our conceptualization of slums is based on information obtained from 50 international domain experts on how they identify and delineate slums from VHR images. The resulting framework is robust enough for global application, though it does still require adaptation to local conditions.

In the expert survey, most respondents referred to countries in Asia and SSA. Thus, it was not possible to integrate knowledge from other regions (e.g. Europe, North America, North Africa, Middle East), and there was also insufficient representation (Table 2-1) for Latin America. The characteristics of slums could be different in these regions (Neuwirth 2005, Dubovyk et al. 2011). Nonetheless, the study does include two world regions with high rates of poverty and slum proliferation (UN-HABITAT 2010). To fully verify the validity and value of the GSO, a larger, more representative survey of experts covering all world regions is required. However, even without such a survey, the usefulness of the current GSO can be tested through its application in different contexts.

The description of general indicators, their attributes and values provides insight into the variability and similarities of slums across the SSA and Asian region. For example, the great majority of respondents from the SSA context specified the roof material in slums to be tin or iron sheets, providing a clear regional characteristic. Irregularity of roads is common for almost all the slums in both regions. Other similarities are the relatively high percentages for a slum site to be close to linear features, an aspect that also explains the elongated shape of slums mentioned by a considerable number of experts. Proximity to 
employment may also be an important driving force for slum development as evident from the responses.

There are also some slight, but noticeable differences between the two regions. A high proportion of respondents specified building type as detached in SSA, whereas the majority of responses from Asia specified the building type as attached, an indication of the higher densities that are generally found in Asian slums. The low percentage of slums located close to hazardous industries in SSA is perhaps more a reflection of the lower rate of industrialization in SSA cities than the importance of this factor. The relatively low percentage of slums close to or inside low socio-economic status neighborhood could be due to the relative lack of formal low income housing; in many SSA cities the slums are the sole source of shelter for low-income groups. Due to the lack of such housing, slums develop and expand informally close to middle or high socioeconomic status neighborhoods (see Kisumu example), where services and informal sector employment may be found. Further work is needed to confirm the validity of such observations.

\subsection{Conclusion}

The generic slum ontology (GSO) provides a set of well-defined attributes that can be used as a starting point for a systematic approach to identify and classify slums. It is useful for the understanding of the relation between image and actual slum characteristics, as it provides the opportunity to integrate local knowledge through the selection of relevant attributes and by adjusting the parameters for each selected attribute to local conditions, as was demonstrated in the Kisumu example. These parameters may be used for practical application in an OOA environment to translate ontology into an operational system of image classification. The GSO can, therefore, be used as a conceptual classification schema as a basis for information extraction from VHR images. In subsequent work we will adapt and apply the generic ontology to other contexts using an OOA. We expect that such an approach will help to remove the ambiguities in slum definition and also guide the classification process in an objective way. The comprehensive knowledge base that can be generated is important for addressing problems related to monitoring slum growth but perhaps more importantly in supporting slum upgrading and improvement. 


\section{Chapter 3}

\section{Transferability of Object-Oriented Image Analysis Methods for Slum Identification ${ }^{*}$}

"Maps cadify the miracle of existence"

- Nicholas Crane

* This chapter is based on the article:

Kohli, D., Warwadekar, P., Kerle, N., Sliuzas, R. and Stein, A. 2013. Transferability of object-oriented image analysis methods for slum identification. Remote Sensing, 5(9), 4209-4228. 


\begin{abstract}
Updated spatial information on the dynamics of slums can be helpful to measure and evaluate progress of policies. Earlier studies have shown that semi-automatic detection of slums using remote sensing can be challenging considering the large variability in definition and appearance. In this study, we explored the potential of an object-oriented image analysis (OOA) method to detect slums, using very high resolution (VHR) imagery. This method integrated expert knowledge in the form of a local slum ontology. A set of image-based parameters was identified that was used for differentiating slums from non-slum areas in an OOA environment. The method was implemented on three subsets of the city of Ahmedabad, India. Results show that textural features such as entropy and contrast derived from a grey level co-occurrence matrix (GLCM), and the size of image segments are stable parameters for classification of built-up areas and the identification of slums. Relation with classified slum objects, in terms of enclosed by slums and relative border with slums was used to refine classification. The analysis on three different subsets showed final accuracies ranging from $47 \%$ to $68 \%$. We conclude that our method produces useful results as it allows including location specific adaptation, whereas generically applicable rulesets for slums are still to be developed.
\end{abstract}




\subsection{Introduction}

The proliferation of slums in cities in developing countries is a major concern for local, national and international organizations. Slum eradication is a major target of the Millennium Project, and has drawn attention to the lack of reliable data for slum areas (UN-HABITAT 2003c, UN-HABITAT 2010). Many slums are not considered to be part of the formal city and are hence not mapped. However, access to reliable spatial and other data on slums is essential for assessing the performance of policies and programs for slum eradication. Such performance-based urban management requires reliable baseline data in order to implement programs for measuring and evaluating policy progress so that required adjustments can be made (McGill 1995).

Census surveys are traditionally used for collecting data on inhabited spaces in most countries every ten years. However, given the dynamic nature especially of urban areas, available data are often outdated. In addition, slums are frequently omitted from formal statistical assessments, hence current spatial information on the concentration or location of slum dwellers is frequently absent (Hofmann et al. 2008, Paar and Rekittke 2011). Other methods, such as participatory approaches, require the involvement of local people and are therefore time-consuming and resource-intensive(Joshi et al. 2002). Remote sensing (RS) based methods can provide spatially disaggregated and regular information on slums by means of effective detection at variable spatial scales (Stoler et al. 2012). With the increasing availability of very high resolution (VHR) satellite imagery, we hypothesize that detection and characterization of slum identification can be improved.

A high level of complexity is involved in the way real-world objects are represented in remote sensing imagery (Blaschke et al. 2008). This, consequently, leads to challenges in image-based detection and characterization (Kohli et al. 2012). Jain (2007) studied the identification of slums in fused Ikonos images. She found that the heterogeneity of an urban environment could not be represented with a pixel-based classification approach using only spectral values. Object-oriented image analysis (OOA), also referred to as object-based image analysis (OBIA)and geographic objectbased image analysis (GEOBIA), has proved to be useful and accurate in urban classification applications (Cleve et al. 2008, Sliuzas et al. 2008b, Ebert et al. 2009, Salehi et al. 2012). Urban areas comprise a mix of land cover features such as water, built-up (buildings and impervious) and vegetation (gardens, parks, agriculture fields). OOA-based methods have the potential to capture this heterogeneity by following a hierarchical procedure for object-classification and by including contextual information for objects and non-physical features (Ebert et al. 2009). In urban studies, this enables identification of buildings, roads, and other anthropogenic features more accurately (Myint et 
al. 2011). Cleve et al. (2008) showed that an object-based classification provides a higher accuracy than pixel-based classification when differentiating urban from rural land-use and land-cover categories. Additionally, with the proliferation of high-resolution imageries and their improved access, automatic object delineation techniques are being developed (Blaschke 2010). Specifically in the case of informal settlements, which can have a relatively complex and undefined morphology, OOA-based methods offer the potential to integrate spectral, spatial and contextual characteristics (Hofmann 2001, Oleire-Oltmanns et al. 2011, Niebergall et al. 2007) for classification. Hofmann (2001) used OOA to classify informal settlements from an Ikonos image. This work was later improved by developing a context-specific ontology to detect informal settlements using Quickbird data (Hofmann et al. 2008). OOA also makes effective use of physical proxies to characterize urban attributes that are not directly observable, for example for the assessment of social vulnerability (Ebert et al. 2009). Proxy variables of vegetation coverage measured from RS images have been used in some studies to derive socio-economic variables (Patino and Duque 2013, Stow et al. 2007, Weeks et al. 2007). A similar approach is attractive for slums and informal settlements because spectral information alone does not allow an unambiguous detection. The reflectance resulting from similar surface material composition or land cover of slum and non-slum areas can be confusing and thus additional criteria are required to avoid misclassifications.

For image-based mapping, it is important to have a clear conceptualization of slums as the objects of interest. A major challenge is that slums have diverse definitions and can have different appearance depending on their geographic setting and local context. This has led to a research gap in slum detection using RS. Previous research on slum/informal settlement mapping focused on developing context-specific methods. Kohli et al. (2012) addressed this gap by developing an ontological framework to conceptualize slums using indicators related to the morphology of the built environment. The generic slum ontology (GSO) provided a comprehensive description of spatial characteristics and their relationships to represent and characterize slums from an image.

This study adapts the GSO to slums in an Indian city, followed by an OOAbased classification. For wider application of a method, it is important to test its applicability in areas of varying characteristics. Earlier studies have shown that rulesets obtained by OOA methods can be transferred and reapplied to various contexts (subsets of same image or different images) (Walker and Blaschke 2008, Hofmann et al. 2011). The transferability is defined as the degree to which a particular method is capable of providing comparable results for other images. A ruleset is easily transferable if it requires minimal manual adaptations for different imaging conditions. This study was carried out with GeoEye-1 image of the city of Ahmedabad, India (Figure 3-2). 
The objectives of this study are as follows:

- Study the applicability of GSO to an Indian city by integrating expert knowledge.

- Based on the knowledge from the local slum ontology, develop an OOA method for classification of slums.

- Explore the transferability of the set of parameters used in the OOA method by applying it to three different image subsets of a single city.

\subsection{Study Area}

Ahmedabad, India's seventh largest city with a population of approximately 5.5 million, covers an area of $205 \mathrm{~km}^{2}$ (COI 2011). The city is divided into an eastern and a western part separated by the Sabarmati river. The eastern part is the Old City and includes the central town Bhadra that is characterized by the barricaded pol system of densely clustered buildings. The Old City is the historical core and is also known as "Walled City". It was found in 1,411 AD and grew over time. The western part includes educational institutions, modern buildings, well-planned residential areas, shopping malls, multiplexes and new business districts. Slum areas are found in both parts and consist of small, selfconstructed building units with roof material of tin, thatch, plastic, concrete or clay tiles (Bhatt 2003), forming small pockets along the river and other parts of the city (Figure 3-1). In 2001, approximately $25 \%$ of the total population of the city lived in slums (COI 2001).

GeoEye-1 images acquired on the 8 January 2010, with a resolution of $0.5 \mathrm{~m}$ panchromatic and $1.65 \mathrm{~m}$ multispectral were used for classification. The images were obtained in standard processing level, i.e., radiometric and geometric correction was done by the image provider. A resolution merge by principal components was performed to produce a pan-sharpened image of four bands with a $0.5 \mathrm{~m}$ resolution ( $\mathrm{Lu}$ and Weng 2005). A vector GIS layer depicting the boundary of the primary river was also used for classification of water. Three subsets of the GeoEye image were used to test the transferability of slum identification parameters between subsets (Figure 3-2). 


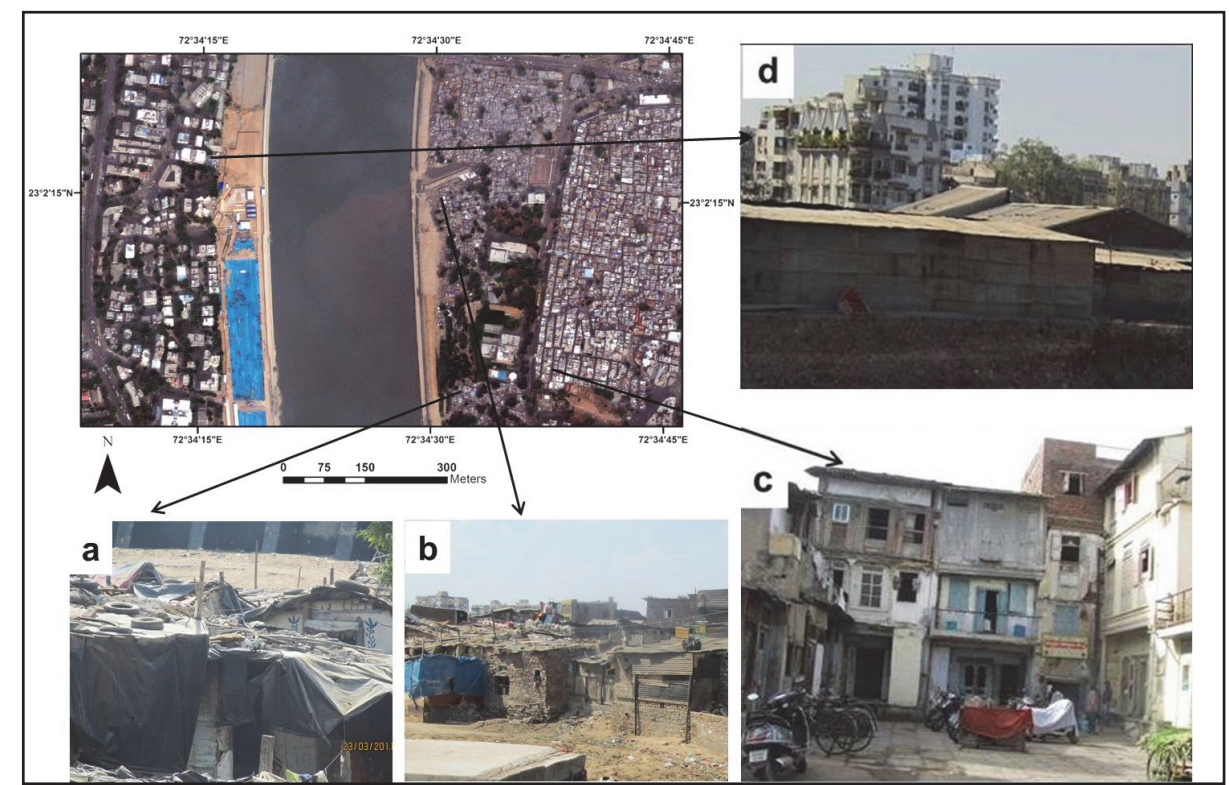

Figure 3-1 Pan-sharpened GeoEye-1 image in true color composite display and ground photographs of different parts of the city: (a) Typical slum house made of non-durable materials. (b) A slum settlement on the east of the river. (c) Old City area. (d) Newly planned residential complexes emerging on the western side of the river.

- Subset-1 is part of the city centre and is approximately $3 \mathrm{~km}^{2}$ in size. It comprises of vegetation, river, residential, commercial buildings and slums, with slums located mainly along the river.

- Subset-2 covers the entire city centre, is much larger in size (approximately $18 \mathrm{~km}^{2}$ ) and includes Subset-1. By means of Subset 2 , effects of enlargement can be studied.

- Subset-3 is part of the outer city and contains a mixture of vegetation, industrial and residential buildings and slums with slums located mainly close to factories. It is of a smaller size than Subset-1 (approximately 1 $\mathrm{km}^{2}$ ) and thus allows studying the effects of transferability. 


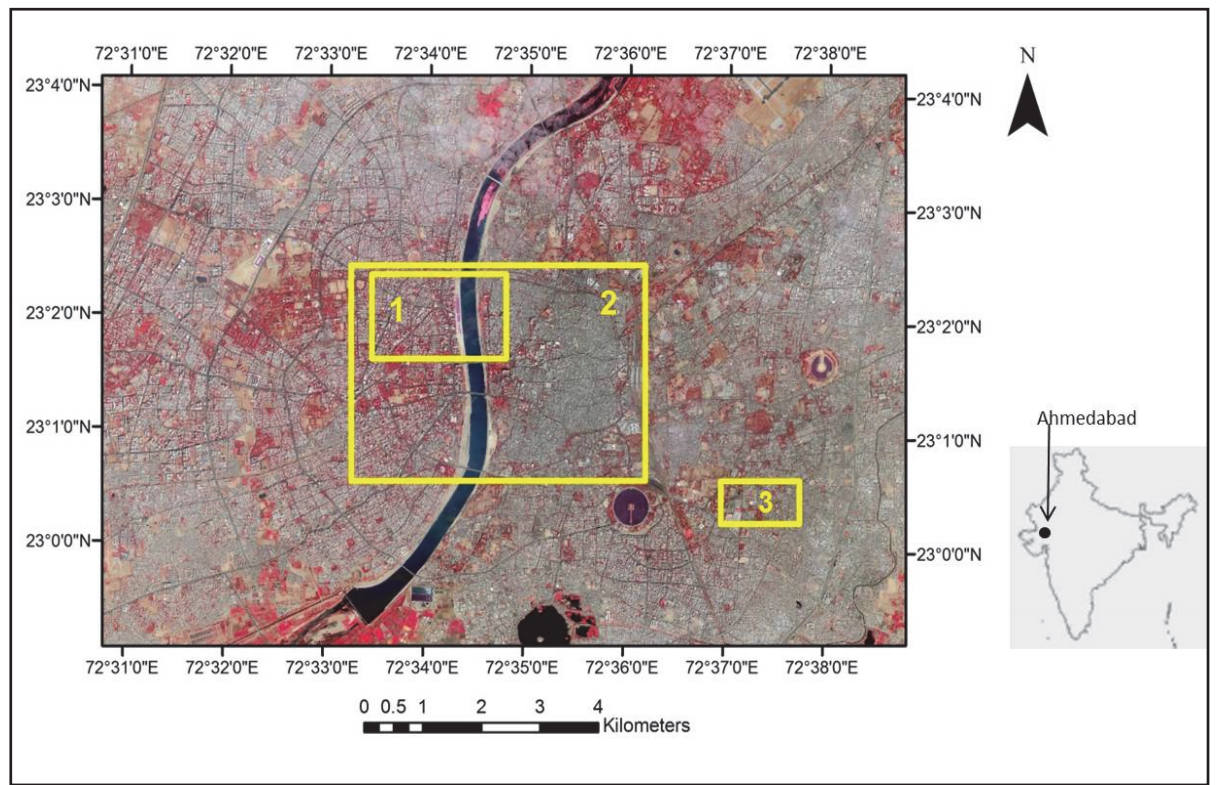

Figure 3-2 Pan-sharpened GeoEye-1 image of Ahmedabad city in color infrared composite display. The three overlaid rectangles mark the extent of different subsets analysed in this study.

In Ahmedabad, buildings are highly variable in terms of size, color, shape, tone and texture. By means of visual interpretation, using parameters such as building roof color, structure, orientation, density as well as on road characteristics, amounts and patterns of vegetation and open spaces, it was observed that there are many types of built-up areas. For example, the planned residential complexes tend to have buildings with bright white roofs that show a well-organized pattern with regular orientation and spacing, and are often separated by planned green spaces or vegetation. Some areas have buildings with grey, whitish grey or brown roofs with variable green spaces between them, and their size and orientation varies. In contrast, buildings in the Old City are relatively old, the oldest ones dating back to 15th century. They show a haphazard pattern with high density, variable orientation, and almost no vegetation and open spaces. In the absence of local knowledge, these buildings may be incorrectly interpreted as slums. Slum detection studies from satellite images need to address the problem of formal-looking informal areas, and informal-looking formal building clusters, as these may lead to severe misclassification. By formal, we refer to areas that are planned by the concerned city authorities. Informal areas generally develop organically, often built by residents themselves without approval from the authorities. Slums are often referred to as informal areas in previous literature (Kohli et al. 2012, Hofmann et al. 2008). The Old City is a good example of informal-looking formal building clusters. It is thus required to study and understand the context in terms of spatial characteristics of different built-up areas. 


\subsection{Method}

This section provides a brief description of a local adaptation of the GSO for Ahmedabad and the methods used to develop an object-based slum classification. The ontology of a domain is defined as a formal representation of all its essential concepts and their relationships (Sowa 2000). The GSO defined by Kohli et al. (2012) focused on morphological indicators of the built environment, specified by the "durable housing" indicator that refers to the structural quality of a house and its location with respect to hazards (UNHABITAT 2003c). The GSO used concepts at three spatial levels: object, settlement and environs. The object level referred to the characteristics of the main physical components of the settlement, i.e., the variables that relate to buildings and roads within a slum (e.g., building size and shape; regularity and width of roads). The settlement level addressed the overall form, shape and density of a slum. Finally, the environs level referred to the location of a slum with respect to surrounding areas in terms of land use and hazard-prone zones. While the GSO is a useful framework to formalize knowledge about complex features such as slums, it is also too general to be immediately useful to parameterize an image classification for a specific setting. Thus, the intermediate step is to adapt the GSO to the specific local conditions of a city.

\subsubsection{Local Ontology of Slums in Ahmedabad}

The GSO indicators were reviewed in relation to the specific morphologies observed in Ahmedabad by means of visual interpretation of imagery and discussions with eight local experts during fieldwork. These local experts were urban planners, NGO employees working in slum upgrading programs, and officials of the Ahmedabad Municipal Corporation (AMC). Relevant indicators were chosen and arranged to form a local ontology for Ahmedabad. Specific values and observations of the indicators referring to each ontological concept are given in Table 3-1. These were used to establish appropriate parameter settings for the OOA. 
Table 3-1 Adaptation of concepts from the generic slum ontology to a local ontology for Ahmedabad followed by respective object-oriented image analysis (OOA) parameterization.

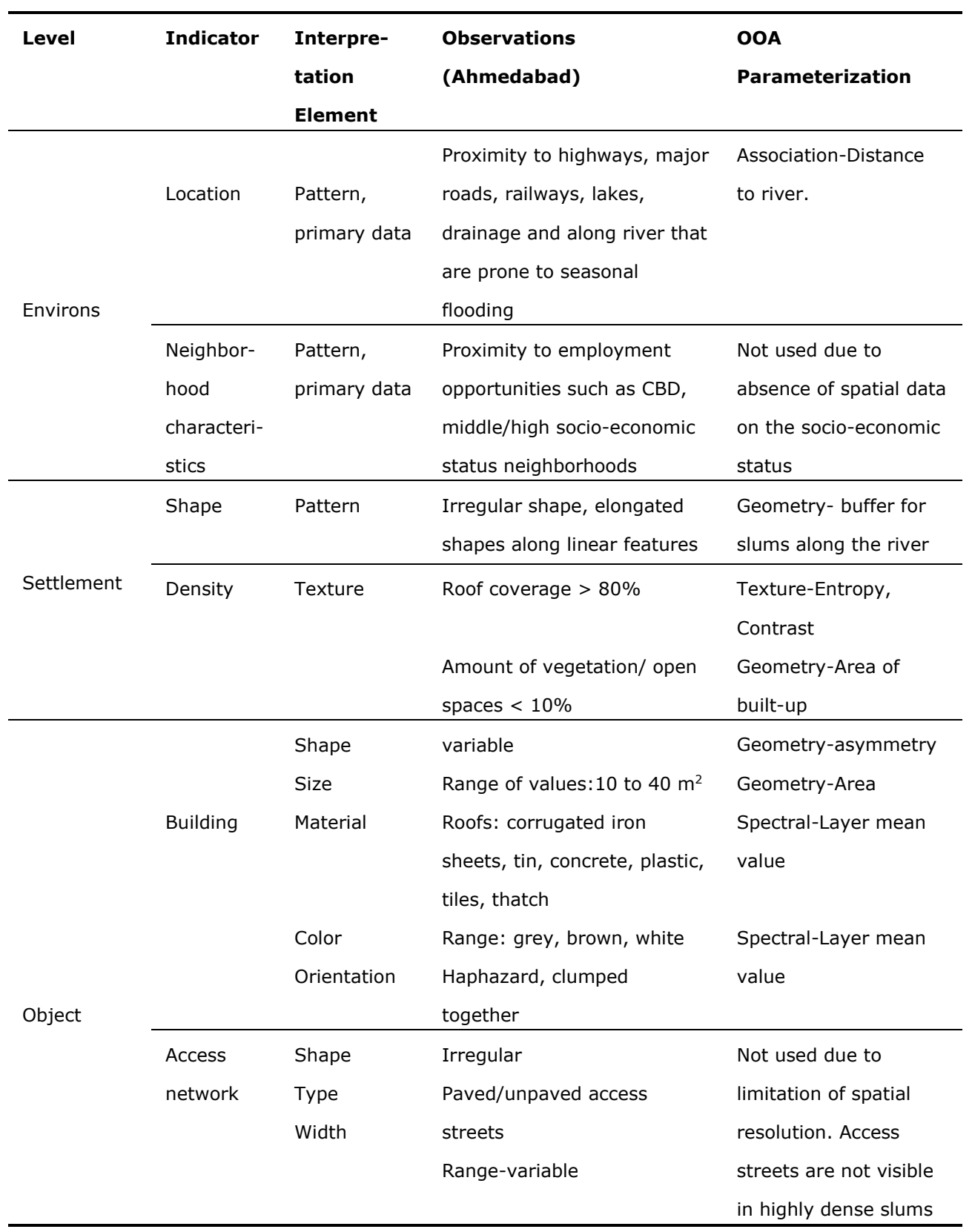




\subsubsection{Converting Local Ontology to OOA Parameters}

The local ontology guided the OOA parameterization by defining the different features for classification. Four broad categories comprising spectral range, geometry, texture and association were chosen to translate the adapted ontology to OOA parameters (Table 3-1). The definitions and details of all the parameters used are given in Tables 3-2 and 3-3 respectively. Conversion of ontological indicators into object-based parameters was done by integrating expert knowledge until object classification was achieved to a visually satisfying degree.

The first step in an OOA is image segmentation, i.e., dividing the image into regions or objects of homogeneous pixel values within the segmented objects. We used multi-resolution segmentation in the analysis. Multi-resolution segmentation is a bottom-up segmentation method based on a pairwise region merging technique (Benz et al. 2004). The first step is to assign appropriate values to the key parameters, namely scale $(\Psi)$, shape ( $\left.W_{\text {shape }}\right)$ and compactness ( $W_{\text {compt }}$ ) to segment objects. Here, $\psi$ determines the maximal allowed heterogeneity of the objects. A large value of $\psi$ results into a low number of relatively large objects, whereas a small value for $\psi$ results in a large number of relatively small objects. The parameter $w_{\text {shape }}$ balances the shape vs. the spectral homogeneity of objects as the sum of two is equal to one. A value of 0.5 was assigned to $\mathrm{w}_{\text {shape }}$ to give equal weight to shape and spectral reflectance. The parameter $w_{\text {compt }}$ determines the compactness of the edges of objects. The values to $\psi, w_{\text {shape }}$ and $w_{\text {compt }}$ are assigned based on expert knowledge and the purpose of classification (Benz et al. 2004). Currently there is no standardized or widely accepted method for optimal scale determination for different application areas and images, though methods such as ESP (Estimation of Scale Parameter (Drăgut et al. 2010) or the POF (Plateau Objective Function (Martha et al. 2011)) have been making the segmentation parameterization process easier and more transparent. In addition, in recent years the initial segmentation, and thus the above parameters, have become less important, with subsequent object shaping procedures allowing more control over the analysis process (e.g., Martha et al. (2012)). Parameter values and segmentation results are shown in Figure 3-3. The segments outlining individual buildings and trees in non-slum areas and clumped buildings as objects in slum areas can clearly be seen (Figure 3-3).

Next, spectral (intensity, brightness), spatial (size, shape, area), textural and contextual features (relation with neighboring objects) of the resulting image objects were analysed (Table 3-2). These features allowed classification of image objects into meaningful classes. A sequence of steps was repeated until all objects were classified. These steps form a ruleset that was then to be applied to the different image subsets (Table 3-3). The settings and adjustments made for each image subset are described below. We have to 
consider this within the usually taken OOA approach to have a common ruleset applicable for every subset. The accuracy assessment is discussed in the results section.

Table 3-2 The definitions of the stable parameters used in the classification of three subsets. For details on each of the parameters, refer to Haralick et al. (1973) and eCognition reference book (Trimble 2014).

\begin{tabular}{|c|c|c|}
\hline Parameter & Description & Definition \\
\hline NDVI & $\begin{array}{l}\text { Normalized difference } \\
\text { vegetation index }\end{array}$ & $\begin{array}{l}\text { An index developed to measure vegetation, } \\
\text { defined as (near-infrared (NIR) - red)/(NIR + } \\
\text { red) }\end{array}$ \\
\hline MG & Mean green & $\begin{array}{l}\text { The mean intensity of all pixels forming an } \\
\text { image object in the green band. }\end{array}$ \\
\hline $\mathrm{RB}(\mathrm{SL})$ & $\begin{array}{l}\text { Relative border to } \\
\text { slums }\end{array}$ & $\begin{array}{l}\text { The ratio of the shared border length of an } \\
\text { image object (with a neighboring image object } \\
\text { assigned to a defined class) to the total border } \\
\text { length. }\end{array}$ \\
\hline GLCM & $\begin{array}{l}\text { Grey level } \\
\text { co-occurrence matrix }\end{array}$ & $\begin{array}{l}\text { Proximal combinations of pixel brightness values } \\
\text { (grey levels) within a particular band of an } \\
\text { image. }\end{array}$ \\
\hline $\mathrm{GLCM}_{E n t}(\mathrm{R})$ & $\begin{array}{l}\text { GLCM entropy for red } \\
\text { band }\end{array}$ & $\begin{array}{l}\text { The measure of orderliness within a band of an } \\
\text { image and relates to textural homogeneity. }\end{array}$ \\
\hline $\begin{array}{l}\operatorname{GLCM}_{C o n}(\mathrm{R}) \text { and } \\
\operatorname{GLCM}_{C o n}(\mathrm{~B})\end{array}$ & $\begin{array}{l}\text { GLCM contrast for red } \\
\text { and blue band }\end{array}$ & $\begin{array}{l}\text { The amount of local variation within a band of } \\
\text { an image. }\end{array}$ \\
\hline Merge & Merge region & $\begin{array}{l}\text { Neighboring image objects of the same class are } \\
\text { merged. }\end{array}$ \\
\hline Area & Area of object & The number of pixels forming an image object. \\
\hline Asym & Asymmetry & $\begin{array}{l}\text { The ratio of the lengths of minor and major axes } \\
\text { of an ellipse approximation of the object. }\end{array}$ \\
\hline ES & Enclosedness by slums & $\begin{array}{l}\text { Image objects that are completely enclosed by } \\
\text { objects belonging to selected class. }\end{array}$ \\
\hline $\operatorname{MDS}(\mathrm{R})$ & $\begin{array}{l}\text { Mean diff. to scene for } \\
\text { red band }\end{array}$ & $\begin{array}{l}\text { Difference between the mean intensity of a } \\
\text { band and all pixels forming an image object in } \\
\text { the same band. }\end{array}$ \\
\hline
\end{tabular}


Table 3-3 Ruleset summary and the quantitative classification criteria used for different subsets. The classification steps show the sequence with top being attempted first.

\begin{tabular}{|c|c|c|c|c|}
\hline $\begin{array}{l}\text { Seq } \\
\text { No. }\end{array}$ & $\begin{array}{l}\text { Classification } \\
\text { Steps }\end{array}$ & $\begin{array}{l}\text { Ruleset-1 for } \\
\text { Subset-1 }\end{array}$ & $\begin{array}{l}\text { Ruleset-2 for } \\
\text { Subset-2 }\end{array}$ & $\begin{array}{l}\text { Ruleset-3 for } \\
\text { Subset-3 }\end{array}$ \\
\hline 1 & Classify Water & $\begin{array}{l}\text { Multiresolution } \\
\text { segmentation } \\
\text { using thematic } \\
\text { layer }\end{array}$ & $\begin{array}{l}\text { Multiresolution } \\
\text { segmentation using } \\
\text { thematic layer }\end{array}$ & No water body \\
\hline 2 & Object creation & \multicolumn{3}{|c|}{ Multiresolution segmentation $\left(\psi=40, w_{\text {shape }}=0.5, w_{\text {compt }}=0.5\right)$} \\
\hline 3 & Classify Vegetation & & NDVI $>0.1$ & \\
\hline 4 & Classify Shadows & MG $<208$ & MG $<122$ & MG $<131$ \\
\hline 5 & Classify Built-up & & $\operatorname{GLCM}_{\text {Ent }}(\mathrm{R})>3.7$ & \\
\hline 6 & Buffer along river & Within 500 Pixels & Within 500 Pixels & No buffer \\
\hline 7 & Classify Slums & $\begin{array}{l}\mathrm{GLCM}_{\mathrm{Con}}(\mathrm{B})<4, \\
\mathrm{ES}, \mathrm{RB}(\mathrm{SL})>0.3 \\
62,000<\text { Area }< \\
63,000\end{array}$ & $\begin{array}{l}2.5>\operatorname{GLCM}_{\mathrm{Con}}(\mathrm{B})< \\
5 \\
\mathrm{ES}, \mathrm{RB}(\mathrm{SL})>0.3\end{array}$ & $\begin{array}{l}5<\operatorname{GLCM}_{\text {Con }}(\mathrm{R})< \\
9 \\
\mathrm{ES}, \mathrm{RB}(\mathrm{SL})>0.3\end{array}$ \\
\hline 8 & Clean-up & $\begin{array}{l}\mathrm{MG}>240, \text { Area }< \\
25,000, \mathrm{ES} \\
\mathrm{RB}(\mathrm{SL})>0.3\end{array}$ & $\begin{array}{l}M G>155, \text { Area }< \\
6,000, E S, R B(S L)> \\
0.3\end{array}$ & $\begin{array}{l}\text { Area }<50,000, \\
\operatorname{MDS}(R)>5, E S, \\
R B(S L)>0.3, \\
\text { Asym }<0.5\end{array}$ \\
\hline
\end{tabular}

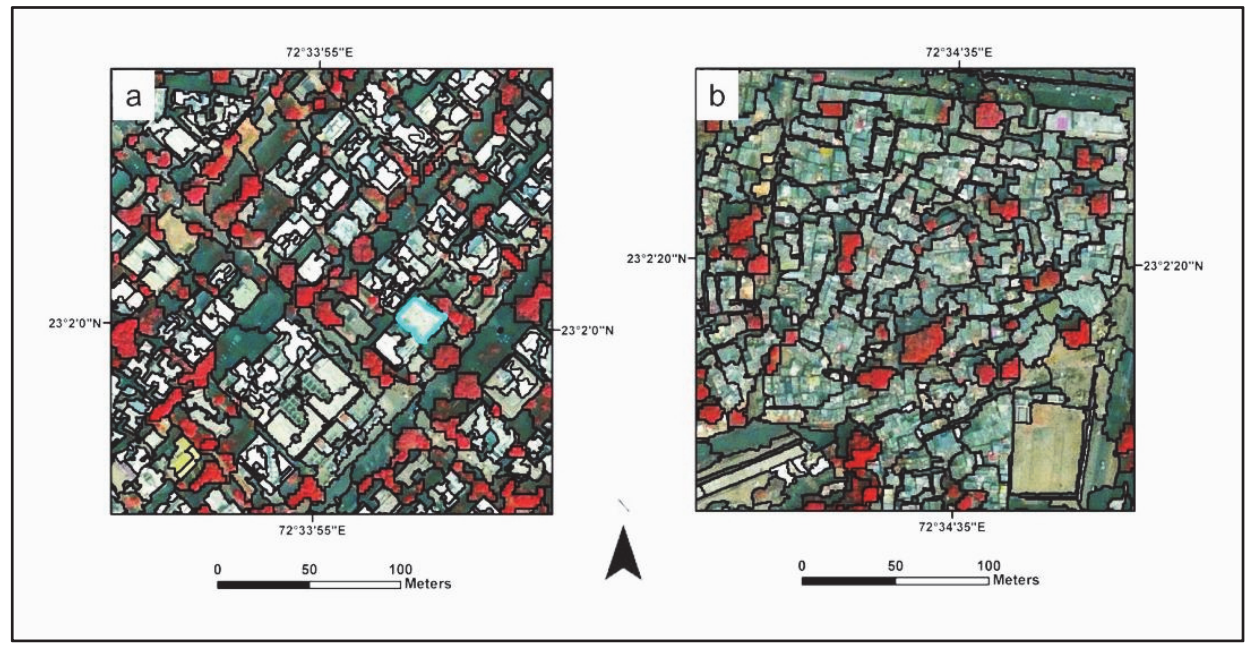

Figure 3-3 Multiresolution segmentation of the multispectral image ( $\psi=40$, Shape $w_{\text {shape }}$ $\left.=0.5, w_{\text {compt }}=0.5\right)$. (a) The individual buildings and trees are outlined in the non-slum areas. (b) In the slum areas, image objects are clumps of buildings due to the relatively low color contrast. 


\subsubsection{Approach for Slum Identification and Classification}

\subsubsection{Subset-1 Part of City Center}

A multi-resolution segmentation was performed to create segments that delineate meaningful objects such as the roofs of buildings and trees (Figure 3-3). Water was classified by using an existing polygon layer of the river. Normalized difference vegetation index (NDVI) values above 0.1 were used to classify vegetation. Shadows correspond to low brightness values in an image and were classified using mean green $(M G)<208$ values. Building roof texture is homogeneous and has high entropy. GLCM entropy values, calculated using Haralick's method (Haralick et al. 1973), discriminated the built-up from other classes. GLCMEnt $(\mathrm{R})>3.7$ was used to classify the built-up area where $\operatorname{GLCM}_{\text {Ent }}(\mathrm{R})$ indicates the GLCM entropy for the red band.

According to the local ontology of Ahmedabad, many slums have developed along the river (Table 3-1). The distance of the Old City from the river, calculated from the top edge of the image, is approximately $250 \mathrm{~m}$. Based upon this knowledge, we defined a buffer of $250 \mathrm{~m}$ on either side of the river for slum classification. This prevented misclassifications in the Old City, which forms a major part of the city centre. The GLCM contrast for the blue band $\left(\mathrm{GLCM}_{\mathrm{Con}}(\mathrm{B})\right)$, enclosedness by slums (ES) and relative border to slums $(R B(S L))$ were used as parameters for slum classification within the buffer (Table 3-3). The GLCMcon(B) values showed different contrasts between slum and non-slum built-up. A GLCM $\operatorname{con}(B)<4$ value corresponded to the relative homogeneity and low contrast within the slum patches. The feature ES was used to classify the misclassified slum-objects embedded within classified slums. For including the missing slums in the buffer, a threshold greater than 0.3 for $\mathrm{RB}(\mathrm{SL})$ was found appropriate. Therefore, built-up and shadow objects with a relative border $>30 \%$ with slums were reclassified as slums. Next, objects of the built-up class were merged and the variable Area was used outside the river buffer to find additional slum patches. In slums areas, due to poor contrast and the resolution that is still relatively large with respect to the size of the slum buildings, individual buildings could not be segmented. This can also be considered a limitation of spatial resolution, as building size in slums is small. The slum buildings were segmented as clumps as compared to the individual building objects in non-slum areas. Thus, a large value of Area could distinguish the slum object from the other built-up objects. Misclassifications were removed by applying additional criteria for refining the classification results as discussed in Section 3.3.4. The sequence of steps was saved as Ruleset-1. 


\subsubsection{Subset-2 Full City Center}

Ruleset-1, developed for the city centre, was applied to the larger Subset-2 area. The values for parameters $\psi$, Wshape and $W_{\text {compt }}$ were unchanged, as was the parameter threshold for detecting vegetation. The threshold for shadow was changed to MG $<122$ as the value of MG < 208 classified the entire builtup as shadow. The previous value of $\operatorname{GLCM}_{\text {Con }}(B)<4$ could only partially classify slum patches inside the buffer and a new threshold range of GLCM $_{\text {con }}(B)$ between 2.5 and 5 was used for classification of slums (Table 3$3)$. The reason for the change in thresholds is the relatively large size of Subset- 2 compared to Subset-1 as a larger subset resulted in a larger number of objects. New threshold values were, as before, decided upon a qualitative evaluation of the resulting classification. The ES and RB (SL) parameter remained unchanged. Area was not used for slum classification in this ruleset, because its inclusion resulted into a large number of false-positives from the Old City. Misclassifications were removed using a clean-up operation. This modified ruleset was saved as Ruleset-2 (Table 3-3).

\subsubsection{Subset-3 Residential and Industrial Part of Outer City}

The morphology in Subset-3 is different compared to that of Subset-1. Subset3 is characterized by large industrial roofs and residential areas in addition to slums. Ruleset-1 was applied to Subset- 3 to check its transferability. Using Ruleset-1, it was possible to classify vegetation. With an unchanged MG value, the whole image was classified as shadow (excluding vegetation). MG values of building objects corresponded to the value range for shadows in Subset-1. The value for shadow classification was decreased $(M G<131)$ and with the same remaining steps, the image had a large number of misclassifications and low accuracy. Thus, the remaining steps of the ruleset had to be adapted by adjusting the parameters. The values for parameters $\psi, w_{\text {shape }}$ and $w_{\text {compt }}$, remained unchanged, as were the thresholds for NDVI and $\operatorname{GLCM}_{E n t}(\mathrm{R})$. Slums were detected using modified threshold values of $\operatorname{GLCM}_{\text {con }}(R)\left(5<\operatorname{GLCM}_{\text {con }}(R)\right.$ $<9$ ) followed by ES and RB (SL) respectively. There were a large number of false-positives from the industrial buildings with long roofs and other built-up areas. These misclassifications were removed using clean-up steps and the new modified steps were saved as Ruleset-3 (Table 3-3). 


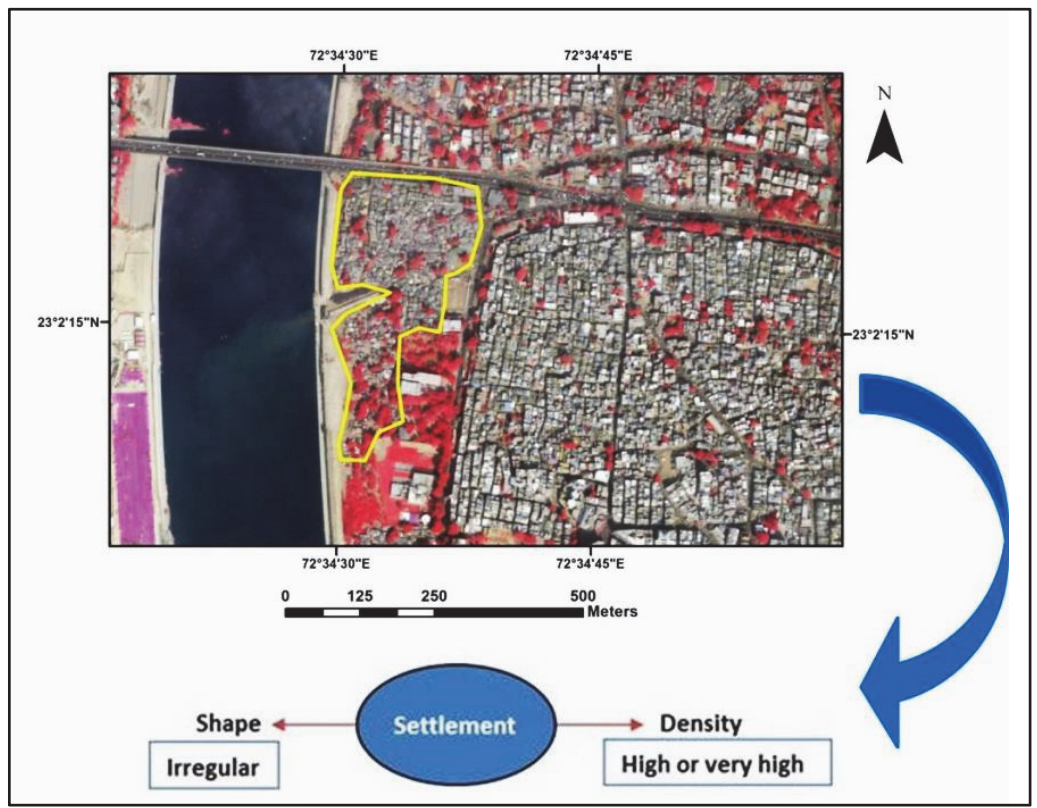

Figure 3-4 Integrating expert knowledge from local ontology of Ahmedabad at settlement level. Slums tend to have an irregular shape with very high building densities in comparison to non-slums.

\subsubsection{Clean-Up}

A clean-up operation was performed to eliminate non-slum objects from the classified slums and reclassify any observed, misclassified slum-objects. For Subset-1, the parameters MG, Area, ES, and RB(SL) were used to remove misclassifications. High values of MG $(>240)$ and low values of Area $(<25,000)$ of merged slum objects were used to remove the false positives from other built-up objects. Subsequently, ES and RB (SL), with the same threshold as earlier ( $>0.3$ ), were used to reclassify the missed slum objects. The same set of parameters was used for Subset- 2 as for Subset-1, whereas values of MG and Area were modified. Non-slum buildings, classified as slums, were reclassified using a lower threshold for MG (>155). Area of objects was further used to remove misclassified built-objects. A lower value of Area $(<6,000)$ was used to preserve the smaller slum patches in this subset. A sequence of steps using the parameters Area, $\mathrm{MDS}(\mathrm{R}), \mathrm{ES}, \mathrm{RB}(\mathrm{SL})$ and Asym, were used to clean up in Subset-3. The parameters used for clean-up, MG in Subset-1, did not improve the classification. This was due to similar brightness of false-positives from non-slum buildings. Subset-3 comprised one large patch of slums. Thus after merging the slum objects, a high value of Area $(<50,000)$ was used to remove smaller non-slum objects. Two new parameters (MDS and Asym) were added in the clean-up of Subset-3. The parameter MDS(R) $(>5)$ could remove misclassified industrial buildings due to relatively high intensity difference in the red band. A low value of Asym can be used to define square objects. Thus, 
Asym $(<0.5)$ was used to distinguish irregularly-shaped slum patch from nonslum built (Table 3-3; Figure 3-4).

\subsubsection{Accuracy Assessment}

To access the accuracy of the classification in the three subsets, we used manually defined samples as reference areas. These areas were delineated by a slum expert from Ahmedabad (Figure 3-5). The accuracy of slums was assessed by using the "error matrix based on training and test area (TTA) mask" in eCognition developer. The error matrix is generated by comparing classification with reference values based on pixels.

\subsubsection{Identifying the Most Stable Parameters}

A main aspect of this study was to provide an investigation into the transferability of rulesets from one area to the next, and from one scale level to the next. In order to do so, we assigned weights to the parameters used for classification depending on the need for adaptation from one subset to another. The purpose of weight allocation was to identify the most stable parameters used for classification. The parameters that required no change were given a bonus weight of 0.25 , resulting in a maximum weight of 1 . Additional weights were given depending upon the applicability of the parameter, change in their thresholds in one, two or all subsets. The significance of specific parameters for classification and weight allocation is shown in Table 3-4.

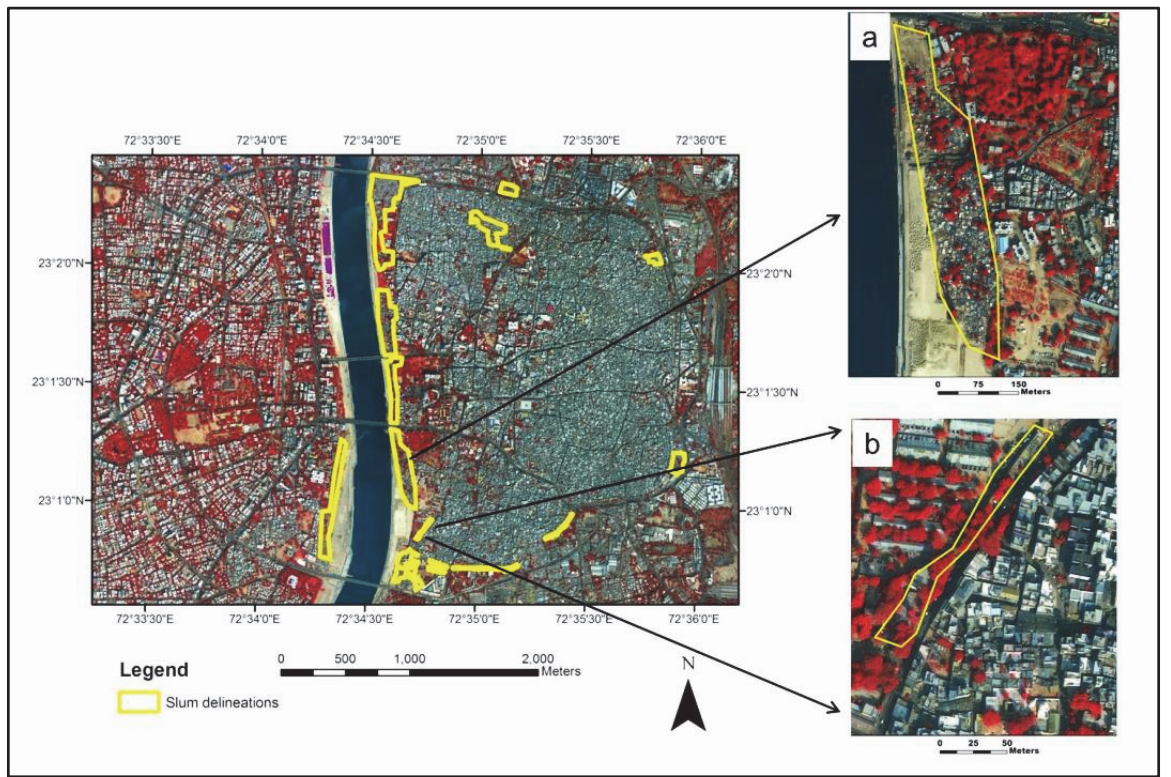

Figure 3-5 Slum delineations by an expert overlaid on Subset-2 and the magnified view of two such delineations: (a) A slum on the bank of the river (b) Slum buildings under the vegetation cover. 


\subsection{Results}

For Subset-1, the parameters GLCMCon(B), ES, RB(SL) and Area were used for classification of slums. The accuracy for slums before clean-up was $56 \%$. Falsepositives were removed by using MG, Area, ES and RB(SL). The accuracy assessment results after clean-up showed an accuracy of $58 \%$ of classified pixels (Figure 3-6). For Subset-2, the same parameters except Area were used for the classification with change in threshold for $\operatorname{GLCM}_{\text {con }}(B)$. The accuracy before clean-up was $38 \%$. A sequence of steps using parameters MG, Area, ES and $\mathrm{RB}(\mathrm{SL})$ was used to clean-up the incorrectly classified slums. The slums in the vicinity of the river were classified with $47 \%$ accuracy after clean-up (Figure 3-7).

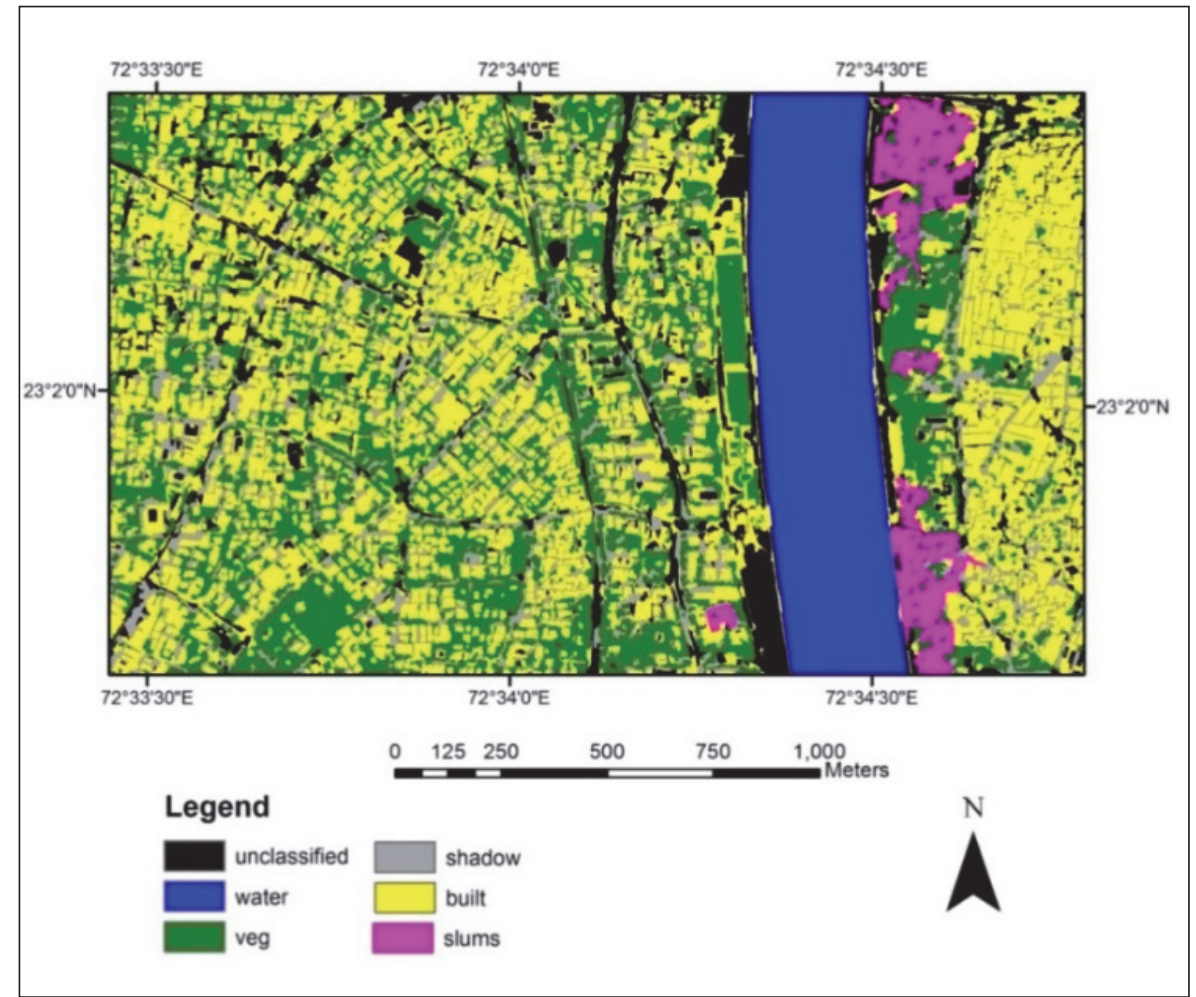

Figure 3-6 Subset-1 classified using ruleset 1 . Slums form a strip close to the east of the river with the exception of one slum patch on the west. 


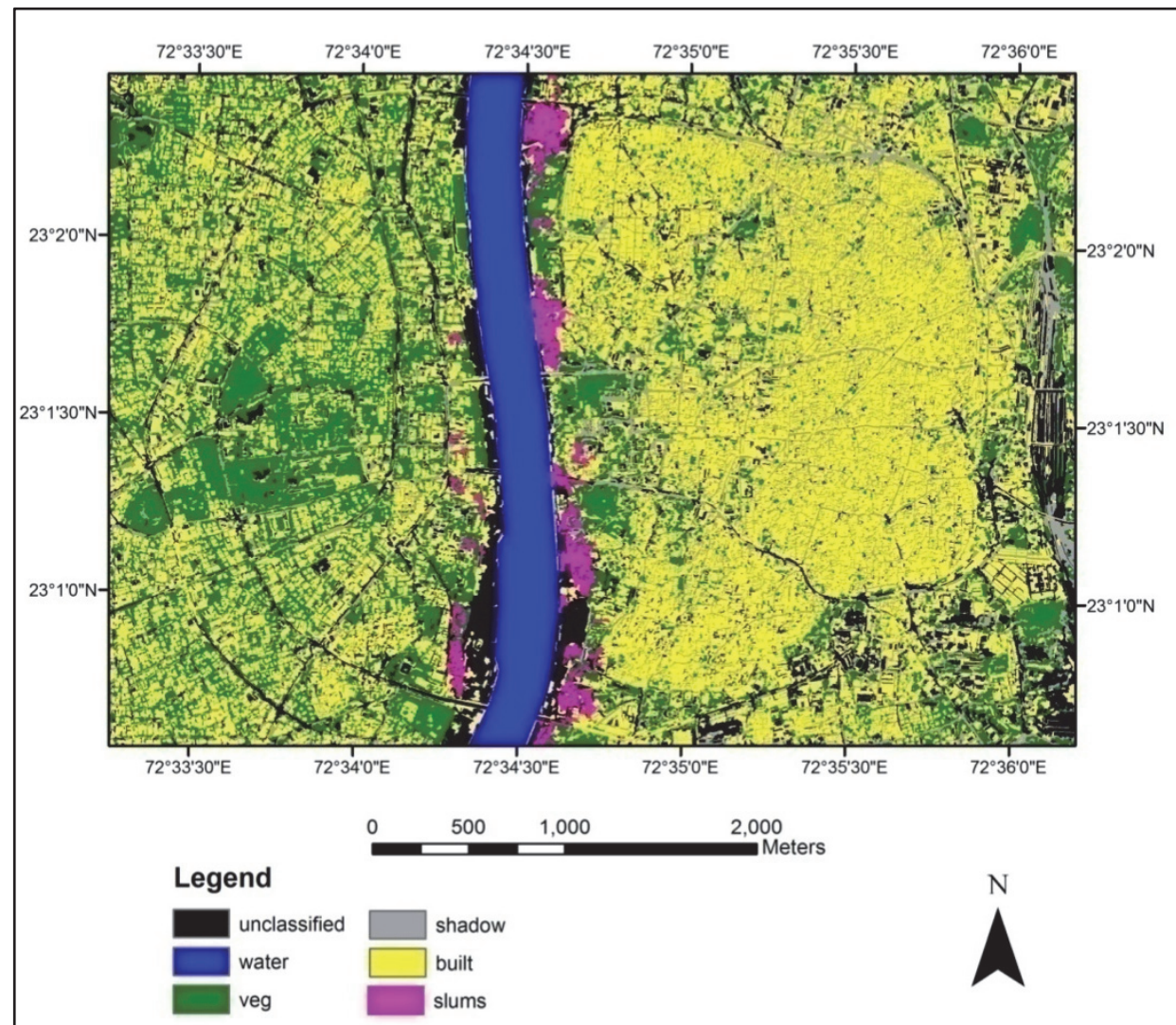

Figure 3-7 Classification of Subset-2 with Ruleset-2 after applying clean-up procedures.

When Ruleset-1 was applied to Subset-3 (with modified MG for shadow classification) (Figure 3-8), the initial results were with a large number of false positives. To improve the classification the $\mathrm{GLCM}_{\mathrm{con}}(\mathrm{R})$ was used with different threshold values. ES and $\mathrm{RB}(\mathrm{SL})$ were used similar to Subset-1. MDS(R) and Asym were new additions in this ruleset for removing false-positives. Subsequently, the remaining slum buildings in the proximity of existing classified slums were reclassified by using $E S$ and $R B(S L)$. Irregular shapes of slums from the ontology could thus be integrated by using Asym to exclude objects misclassified as slums (Figure 3-4). With Ruleset-3, slums in Subset-3 were classified (see Figure 3-8) with an accuracy of $68 \%$. 


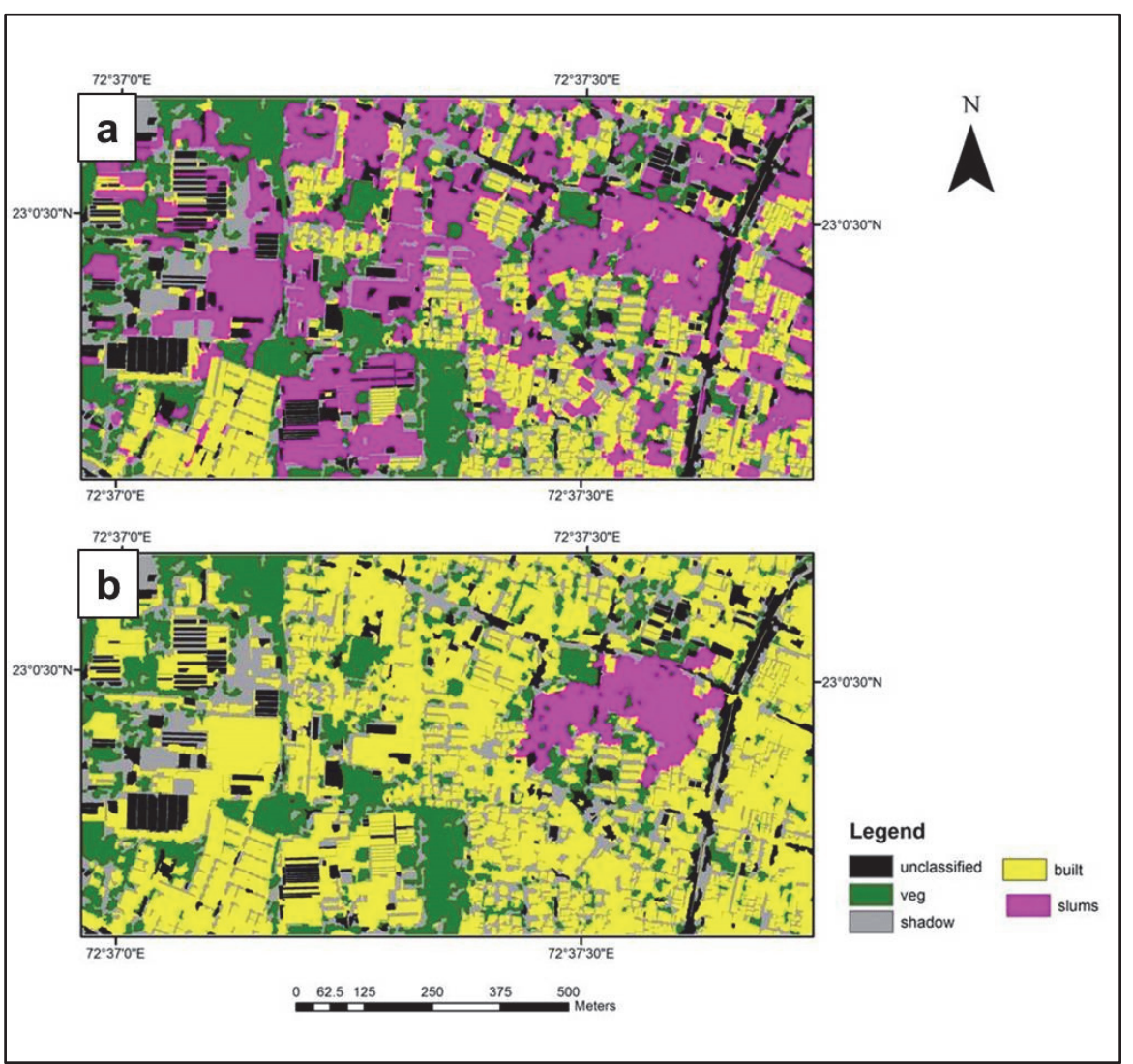

Figure 3-8 Classification results of Subset-3: (a) Application of Ruleset-1, industrial buildings are misclassified as slums due to their large roofs. (b) Application of Ruleset3 , the slum area is now correctly classified after removing the false positives.

\subsubsection{Identifying the Most Stable Parameters}

Some parameters (in italics in Table 3-4) were stable and could be used with some or no modification. To evaluate the stability, weights were assigned to parameters based on their applicability on different subsets. For example, the NDVI was used to classify vegetation in all three subsets with the same threshold. A bonus of 0.25 was given in such cases where the same parameter was applied to all subsets with the same threshold. Alternately, the same parameter could be used to classify features only after modifying thresholds. For example, MG could be used to classify shadow with changed thresholds in all subsets. A bonus of 0.2 was given to the parameter in such cases. There were other parameters such as $\operatorname{GLCM}_{\mathrm{Con}}(\mathrm{B})$ (Table 3-4), which worked on two subsets but not in the third. In this case, a bonus of 0.1 was assigned. NDVI, $\mathrm{GLCM}_{\text {Ent }}(\mathrm{R}), \mathrm{ES}, \mathrm{RB}(\mathrm{SL})$ followed by $M G$ received the highest weights according to their stability (Table 3-4). For clean-up, ES, RB(SL) and Area were the most important parameters. Other parameters were useful but their applicability 
depended on the particular subset characteristics. For example, MDS(R) and Asym were useful for the specific morphology of Subset-3.

Table 3-4 Allocation of weights to the parameters depending on applicability in different subsets.

\begin{tabular}{|c|c|c|c|c|c|c|}
\hline Class & Parameter & Subset-1 & Subset-2 & Subset-3 & Bonus & Total \\
\hline Vegetation & NDVI & 0.25 & 0.25 & 0.25 & 0.25 & 1 \\
\hline Shadow & MG & 0.25 & 0.25 & 0.25 & 0.2 & 0.95 \\
\hline Built-up & $G L C M_{E n t}(R)$ & 0.25 & 0.25 & 0.25 & 0.25 & 1 \\
\hline \multirow[t]{4}{*}{ Slums } & $\operatorname{GLCM}_{\text {con }}(B)$ & 0.25 & 0.25 & 0 & 0.1 & 0.6 \\
\hline & $\operatorname{GLCM}_{\text {con }}(\mathrm{R})$ & 0 & 0 & 0.25 & 0 & 0.25 \\
\hline & $E S$ & 0.25 & 0.25 & 0.25 & 0.25 & 1 \\
\hline & $R B(S L)$ & 0.25 & 0.25 & 0.25 & 0.25 & 1 \\
\hline \multirow[t]{6}{*}{ Clean-up } & MG & 0.25 & 0.25 & 0 & 0.1 & 0.6 \\
\hline & Area & 0.25 & 0.25 & 0.25 & 0.2 & 0.95 \\
\hline & $E S$ & 0.25 & 0.25 & 0.25 & 0.25 & 1 \\
\hline & $R B(S L)$ & 0.25 & 0.25 & 0.25 & 0.25 & 1 \\
\hline & $\operatorname{MDS}(\mathrm{R})$ & 0 & 0 & 0.25 & 0 & 0.25 \\
\hline & Asym & 0 & 0 & 0.25 & 0 & 0.25 \\
\hline
\end{tabular}

\subsection{Discussion}

The advent of VHR satellite imagery has resulted in a surge of RS-based urban studies in a variety of areas (Herold et al. 2003). A number of these studies have focused on cities of developing countries. Most of the earlier urban models and methods were developed in and for a western context. There have been successful attempts to apply these models by adapting them to different contexts. The widespread use of landscape metrics in urban applications, which were primarily developed for American cities [36], is such an example. Nonetheless, there may be problems in adapting such approaches to the developing context. An example is the studies on determining socio-economic status of residential areas using spatial patterns and configuration, such as the vegetation, impervious surface, bare soil (V-I-S) model proposed by Ridd (Ridd 1995). The V-I-S model is used to infer land use depending on the proportional abundance of each of the land cover data. This approach may well work in most western cities but has limitations in a developing setting such as India.

For image-based analysis, it is thus important to understand the processes underlying such complexities. Slum identification and classification pose an additional challenge, because of differences in definition and appearance within and among contexts. The relatively low number of international publications on RS-based slum/informal settlement identification in India, despite the 
acknowledged scale and importance of the problem, substantiates the challenges involved in this area (Jain 2007, Kit et al. 2012). Our research is an attempt to take a step forward in this direction by using a systematic classification approach using an OOA guided by locally adapted generic slum ontology (Kohli et al. 2012). The use of local ontology helped us to integrate expert knowledge during classification. Slum features typically used by experts in visual interpretation and delineation served as a starting point for the application of the slum ontology. This knowledge integration is important to understand the characteristics of slums in a city. We developed a ruleset in OOA to differentiate slum from non-slum areas and explored its applicability on subsets of varying characteristics. This was different from developing three distinct rulesets independently, as it was carefully checked on which parameters changes were necessary when applying the ruleset to the other subsets. Our analysis showed that the parameters NDVI, MG, and GLCMEnt $(R)$ were useful for basic classification of vegetation, shadow and built-up, respectively, in all three subsets. For classification of slums, GLCMcon(B), ES and $\mathrm{RB}(\mathrm{SL})$ were stable and are thus transferable parameters. Area, in terms of number of pixels, was suitable for eliminating false positives in all subsets. ES and $\mathrm{RB}(\mathrm{SL})$ were uniformly useful for reclassifying any misclassified slum objects embedded within the slums.

We used the same set of parameters to explore the scope for creating a generic ruleset that could be applied throughout the city irrespective of location and scale. Variability of the urban environment is such that a generic ruleset could not be defined. This variability is caused by the composition of different builtup areas. For example, Subset- 3 comprised industrial structures in addition to residential buildings. The ruleset had to be modified to address this by using spectral value (MDS) and shape (Asym) parameter to differentiate slums. In addition, the use of different thresholds and parameters for clean-up (Table 34 ) is due to scene-specific adaptations. In the future, it may be a solution to have a generic definition of area types like industrial or old city and applying that to the scene to divide it into sections that could be further processed with a specific ruleset.

The same basic approach can most likely be applied to a different context with local adaptation. Once the ruleset has been finalized an efficient monitoring of slums over time (e.g., size, density, changes in road network characteristics) becomes possible, though rulesets may have to be adapted to new situations even in the same area. A further alternative to our approach based on explicitly encoded expert knowledge may be to incorporate training data based on known slums, and the use of machine learning tools to identify the relevant parameters that define those urban features. 
Most Indian cities are complex, in terms of morphology and have a historical significance to their development. For instance, Ahmedabad, like many other Indian cities, has an old city core known as the "Walled City" which developed over 6 centuries ago. During our field visit to Ahmedabad, many buildings in the Old City were found to be dilapidated and precarious, and effectively constituting slum buildings (Figure 3-1). After discussion with one of the local experts, we found that the architecture and planning in the Old City was predesigned to promote community living and security in case of communal riots. Based on the cultural and historic importance of the Old City, the Archaeological Survey of India (ASI) has nominated it for Unesco's world heritage list (UNESCO 2011). The unique architecture comprises "Pol housing", typically consisting of closely packed houses with a common backyard for people from one community (Figure 3-1). In the absence of detailed ground knowledge, it is difficult to differentiate these congested areas from slums in the imagery. This was a limitation of RS-based slum identification and, thus, required a customized approach. We used expert knowledge integrated in the slum ontology to address this problem. Creation of buffer areas on either side of the river reduced the area of analysis for Subset-1 and 2, reducing the misclassifications from the Old City. The possibility of missing slums within the Old City remains, but this is more of a problem of slum definition. Our method does provide a set of stable parameters that worked on subsets of varying characteristics in an Indian city. It may work well if there are no slums outside the river buffer, but care must be taken not to use such an approach in an area with completely different characteristics.

The accuracy values for the three subsets range from $47 \%$ to $68 \%$ (after cleanup). In the absence of reliable data, the delineations of slums by a local planner were used as reference (Figure 3-5). However, the question remains whether such reference data are a suitable benchmark for accuracy assessment. The amount of generalization an expert introduces in delineating can impact the accuracy results substantially, while the conceptualization used to define slums may also differ. However, in a data poor environment, this approach was the best-suited to get an unbiased validation. This problem is accentuated by the fact that there are political, administrative as well as physical aspects related to slum definitions. An inherit problem of vegetation cover hindering the detection of slum buildings also remains (Figure 3-5). Considering that only morphological indicators derived from images were used for classification, the accuracy results are acceptable. Additional information in the form of GIS layers may be helpful in refining the results further. For example, slum areas tend to have community water connections instead of individual taps in each household. If detailed data on water connections are available, it can be integrated with population of a settlement. A scenario with settlements of dense population with few water connections could then be used to find probable slums. Similarly, data on other utilities such as electricity or gas 
connections may also be useful. Such additional information may also address the problem of distinguishing formal-looking informal areas, and informallooking formal building clusters. For example, the road-side shops and markets in many Indian cities have structural similarity to slum areas. We recommend such investigation for future research in slum identification studies.

\subsection{Conclusions}

Slum detection studies need to incorporate the issue of variable definitions and appearance of slums in different contexts. To include contextual knowledge, we use expert knowledge encoded in an ontological framework to classify slums in the city of Ahmedabad, India. Using the generic slum ontology as a basis, we tested the transferability of an Object-oriented image analysis (OOA) method for slum classification developed on one image area to other image areas. Our analysis identified textural features derived from a grey level cooccurrence matrix (GLCM) such as entropy (GLCMEnt) and contrast (GLCMcon), relation with classified slum objects, in terms of enclosed by slums (ES) and relative border with slums (RB) and the size of image segments (Area) as stable parameters for classification of built-up areas and slum identification. These parameters worked on all the subsets with minimal adaptation and thus, can be considered as most transferable. The analysis on three different subsets shows accuracies ranging from $47 \%$ to $68 \%$. The applicability of our OOAbased detection method in other cultural spheres remains to be tested. 


\section{Chapter 4}

\section{Urban slum detection using texture and spatial metrics derived from satellite imagery ${ }^{*}$}

"Knowing where things are, and why, is essential to rational decision making

Jack Dangermand

* This chapter is based on the article:

Kohli, D., Sliuzas, R., Stein, A. 2013. Urban slum detection using texture and spatial metrics derived from satellite imagery. Journal of Spatial Science (Undergoing revision). 


\begin{abstract}
Slum mapping is challenging due to the variability in slum types and definitions. This research aimed at developing a method for slum detection from very high resolution images based on the morphology of the built environment. It consists of segmentation followed by hierarchical classification using an object-oriented image analysis and integrating expert knowledge in the form of a local slum ontology. Results show that textural feature contrast derived from a grey level co-occurrence matrix was useful for delineating segments of slum areas or parts thereof. Spatial metrics such as the size of segments, proportions of vegetation and built-up were used for slum detection. The percentage of agreement between the reference layer and slum classification was $60 \%$. This is lower than the accuracy achieved for land cover classification $(80.8 \%)$, due to large variations. We conclude that the method produces useful results and has potential for successful application on contexts with similar morphology.
\end{abstract}




\subsection{Introduction}

An important consequence of urbanization in developing countries is the high growth rate of urban poverty. Urban poverty is manifested most clearly in the proliferation and expansion of slums, home to a growing proportion of urban dwellers. According to UN-HABITAT (2010), the world's slum population is expected to reach 889 million by the year 2020. Although many improvements have been made in the living condition of many slum-dwellers as a result of local and global efforts, their number is still estimated to grow by an average of 6 million every year (op cit). Regularly updated information on slums is therefore needed to guide policies and programs aimed at interventions and improvements (UN-HABITAT 2014). Quantification of slum estimates by identifying and defining slums spatially in a consistent manner is required to be able to use geographical targeting for slum intervention programs (Sliuzas et al. 2008a).

In remote sensing (RS), object-oriented image analysis (OOA) methods have been shown to provide improved analyses of urban areas (Cleve et al. 2008, Ebert et al. 2009, Myint et al. 2013, Taubenböck and Kraff 2014). Such methods are also referred to as object-based image analysis (OBIA) or geographic object-based image analysis (GEOBIA). OOA has great potential for informal settlement mapping because of its ability to include spatial, spectral and contextual characteristics similar to human cognitive image interpretation. This approach integrates expert knowledge in the classification process, which is a major advantage over conventional pixel-based approaches (Hofmann 2001, Haala and Brenner 1999, Kohli et al. 2013, Hofmann et al. 2008). Image segmentation in combination with a hierarchical object-based classification has been used for mapping residential land use and identifying socioeconomic status from very high resolution (VHR) imageries (Stow et al. 2007). Examples include studies on determining socio-economic status of residential areas using spatial patterns and configuration, such as the vegetation, impervious surface, bare soil (V-I-S) model proposed by Ridd (Ridd 1995, Weeks et al. 2007).

In this study, we explore the potential of using the V-I-S model in an Indian context, the city of Pune, to identify and classify slum areas. A combination of texture and spatial metrics is used to quantify different land cover features in an OOA environment. The proportions of the constituent land cover classes i.e. vegetation and impervious, serve as proxies for the identification of slums. Previous research has shown that inclusion of contextual knowledge encoded in an ontological framework can be used for image-based classification (Kohli et al. 2013, Belgiu et al. 2014b). Use of the generic slum ontology (GSO) based upon indicators related to the morphology of the built environment helps to address the issue of variable definitions and appearance of slums in different contexts (Kohli et al. 2012). This study adapts the GSO to slums in Pune and 
then follows an OOA-based classification considering two spatial levels. The objectives are as follows:

- to determine the applicability of the GSO to the Indian city of Pune.

- to explore the use of texture and spatial metrics to quantify the spatial patterns at different ontological Levels.

- to test the methodology of slum identification based on the V-I-S model.

Previous research applied the V-I-S model to infer land use from the proportional abundance of each of the land covers (Herold et al. 2002, Herold et al. 2003). These studies, however, were mainly carried out for western cities. Similar studies, dealing with slum identification as well, were done for the African city of Accra (Weeks et al. 2007, Stow et al. 2007, Stow et al. 2010, Stoler et al. 2012). To the best of our knowledge, such studies do not exist for Indian cities. With their complex morphologies that are a result from the organic growth or their historical development, Indian cities pose a challenge for slum studies. Slum identification itself is already a complicated task considering the variability in appearances and definitions (Sliuzas et al. 2008a). The aim of this paper is to detect Indian urban slums using texture and spatial metrics derived from satellite imagery. This is done by combining the GSO, the V-I-S model and an OOA.

\subsection{Definition of slums}

Various reasons might explain the occurrence of slums in different cities of the world. Slums tend to share some common features globally (Patino and Duque 2013). For instance, most slums are characterized with a high concentration of poor people, substandard shelter and poor physical environmental conditions. These characteristics also date back to the slums of medieval cities in the western world (Haala and Brenner 1999, Neuwirth 2005). Over time, different countries have developed national and in some cases, local definitions of slums. In order to work towards monitoring slums world-wide, a global definition of a slum household was formulated as one lacking in any one of five factors: secure tenure, access to safe water, access to sanitation, sufficient living area and durability of housing (UN-HABITAT 2006). Kohli et al. (2012) developed an ontological approach to conceptualize slums using the durable housing indicator that is most relevant for RS-based slum identification and classification. In that study, the authors conducted a survey to find the most common physical features of slums concerning the structural quality of housing, infrastructure and location. These features were compiled to form the GSO that may be adapted to different contexts. The GSO comprises indicators related to the morphology of the built environment at three spatial levels: 
object, settlement and environs levels. It provides a comprehensive description of spatial characteristics and their relationships to characterize slums in a very high resolution (VHR) satellite image. In this study, we used the GSO as the basis for classification by adapting it to Pune, India.

\subsection{Materials and methods}

\subsubsection{Study area}

Pune is one of the fastest developing urban agglomerations in Asia and ranks eighth at national Level in India (COI 2001). It covers an area of $244 \mathrm{~km}^{2}$ and is considered the cultural and educational centre of Maharashtra State in India. Its population has grown significantly over the past two decades. Favorable geographic location, pleasant climate, immense employment opportunities and improved educational facilities are some of the reasons attributed to high migration of people from other parts of the country. From 1981 to 1991, the population grew by $30.2 \%$, and between 1991 and 2001 by $62.2 \%$ (Shekhar 2010). With approximately 1 million slum dwellers out of the total population of about 3.15 million (MASHAL 2011), India's Town and Country Planning Organization (TCPO) ranks Pune third in cities with largest number of slums in India.

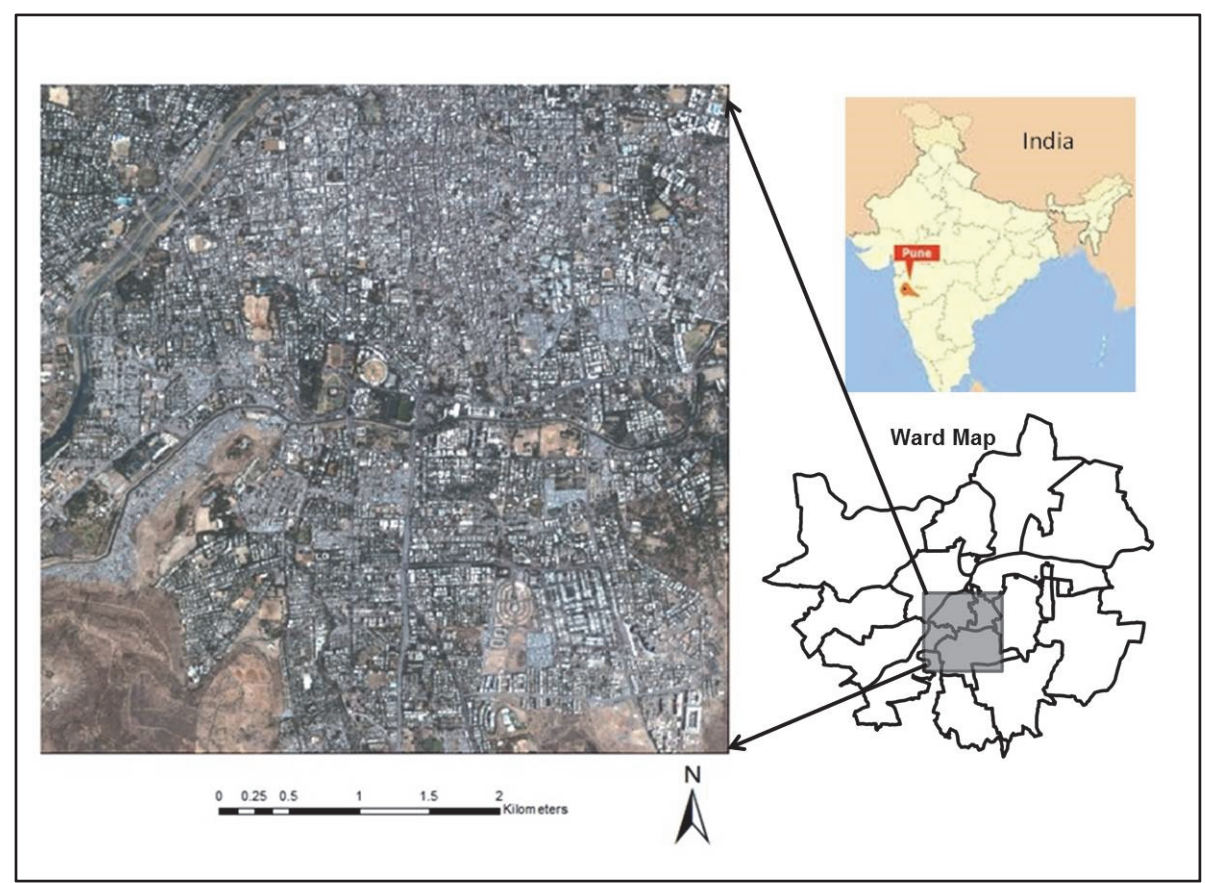

Figure 4-1 Location map of the study area in the South-Western part of India. The ward map shows the administrative wards of Pune city. The pan-sharpened Quickbird image is from the central part of the city. 


\subsubsection{Image data}

A cloud-free pan-sharpened Quickbird satellite image ( $0.6 \mathrm{~m}$ resolution) of red, green and blue bands of the year 2006 was used for this research. The reason for using a relatively old image was its availability and that it was also reasonably close to the acquisition period (2006 to 2008) of the slum atlas reference data available from Maharashtra Social Housing and Action League (MASHAL), a local NGO. The image scene covering the central part of the city was selected for analysis (Figure 4-1). This scene is representative of the urban form of the entire city as it contains most of the land use types found in Pune. Roads and water bodies were digitized from the image and were also used in analysis. For accuracy assessment, a polygon layer of the slum pockets, provided by MASHAL was used as reference data (Figure 4-2). According to their survey, a total of 477 slum pockets were identified in Pune city. Approximately $33 \%$ of the total population of Pune lives in only $2.34 \%$ of the total land of the city. Many of the slums are located on hill slopes, along rivers and other environmentally-sensitive areas (MASHAL 2011). According to Pune's disaster management plan (PMC 2012), slums are most vulnerable in case of disasters such as floods, earthquakes and landslides. Given their vulnerability, it is important to update information on slum dynamics to monitor and potentially regulate their further growth. Updated spatial information can also help in crisis management as many slums of Pune lie in hazardous areas (Shekhar 2010).

\subsubsection{Local ontology of slums in Pune}

A major challenge in slum identification from satellite images is the morphological resemblance of slums to non-slum areas (Kit et al. 2012, Kuffer et al. 2014). Prior knowledge on the spatial pattern and context is thus useful in detecting slums in a particular urban environment. To do so, the GSO has been adapted to the local conditions of Pune slums. Relevant indicators are chosen and arranged to form a local slum ontology. Specific values and observations of the indicators referring to each ontological concept are given in Table 4-1. The table shows the specification of physical characteristics adapted from the generic ontology used for identification and delineation of slums, from environs level through settlement level to object level. The characteristics of slums in Pune were observed by visual interpretation and ground knowledge. Ground data collection included visits to some slum settlements and discussion with experts working for slum related issues. The observations at three spatial levels of ontology are as follows:

- At environs level, slums in Pune tend to be located on steep slopes, floodprone and marshy areas, along canals, railway tracks and major roads. These locations are disaster-prone and generally unattractive for planned development because of potential risks. Slums are also found close to 
areas that offer employment opportunities such as the central business district (CBD), industrial areas and middle/high socio-economic status neighborhoods.

- At settlement level, most slums are highly compact, displaying a roof coverage of more than $70 \%$ and vegetation/open spaces comprising less than $20 \%$ of the settlement. The shape of the slums is often irregular, except for those along canals and roads that tend to have a linear shape.

- At object level, the small size of buildings, variable roof materials, the irregularity, narrowness and unpaved surface of roads were important observations for slum identification.

The above observations have been categorized and analysed based on the ontological framework. The relevant indicators referring to each ontological concept are further assigned specific OOA parameters (Table 4-1).

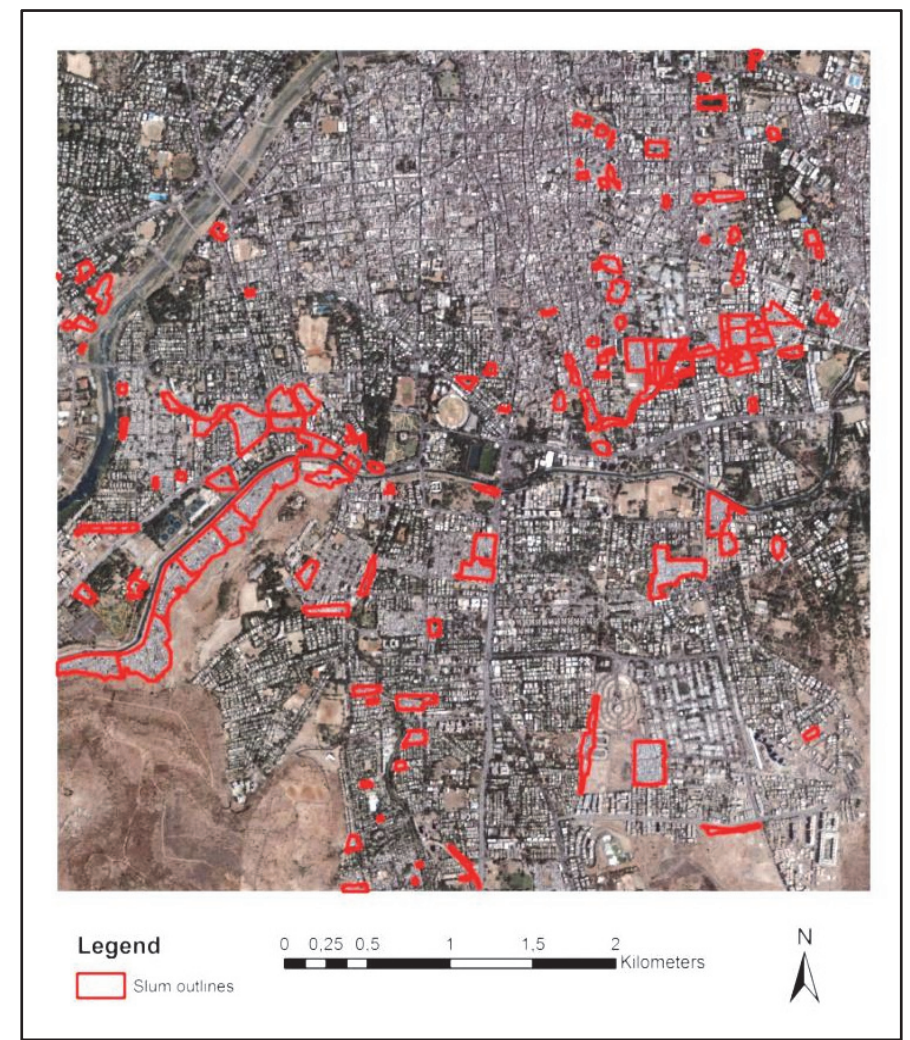

Figure 4-2 Slum delineations provided by a local NGO (MASHAL) displayed on the Quickbird image of Pune city. 
Table 4-1 Adaptation of GSO concepts to an ontology for Pune with OOA parameterization at environs, settlement and object levels.

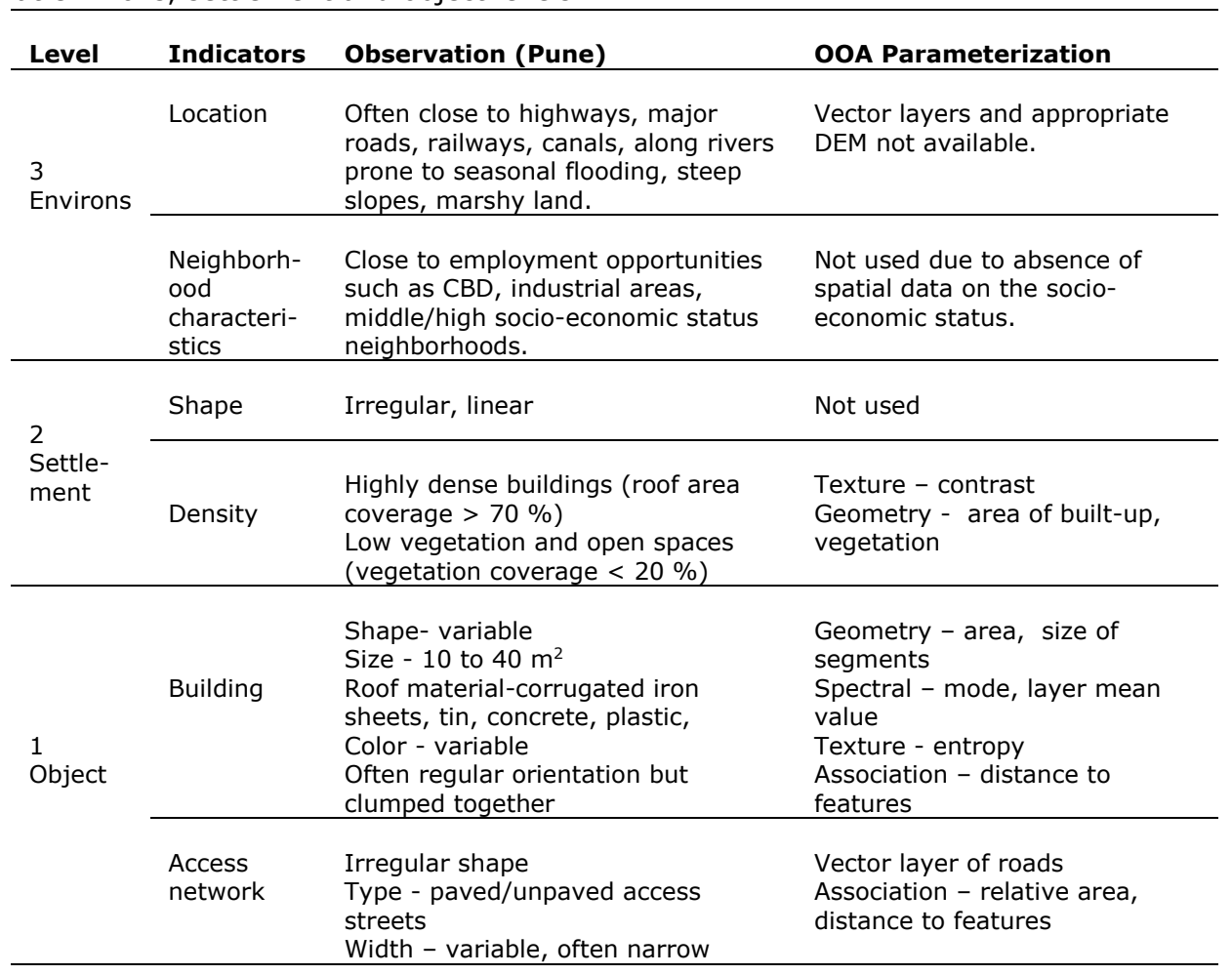

\subsubsection{Conversion of local ontology to OOA parameters}

OOA parameterization was guided by the local ontology which defines the different features for classification. Conversion of ontological indicators into object-based parameters was done qualitatively by integrating expert knowledge until object classification was achieved to a visually satisfying level. Spectral range, geometry, texture and association were mainly chosen to translate the local slum knowledge into specific OOA parameters. The translation of ontological concepts from object to environs level is shown in Table 4-1. This study focuses at the settlement and object levels, being the most relevant with respect to the high resolution image.

The first step in OOA is image segmentation, i.e. dividing the image into regions or objects of homogeneous pixel values within segmented objects. Multi-resolution segmentation is a bottom-up segmentation method based on a pair-wise region merging technique. The size and constituents of segments are controlled by assigning appropriate values to the key parameters, scale $(S P)$, shape ( $\left.W_{\text {shape }}\right)$ and compactness ( $\left.W_{\text {compt }}\right)$ to segment objects. For definitions of these parameters, refer to Kohli et al. (2013) or the standard reference source (Trimble 2014). A value of 0.5 was assigned to $w_{\text {shape }}$ to give 
equal weight to shape and spectral reflectance. Expert knowledge, driven by purpose of classification, is used to decide upon the values of $S P, W$ shape and $W_{\text {compt }}$ (Baatz and Schäpe 2000). Two bottom-up hierarchical segmentations representing settlement level (Level 2) and object level (Level 1) were implemented (Figure 4-3).

At Level 1, image object primitives were generated representing the basic land cover classes such as built-up and trees. The built-up class includes building roofs and other impervious materials such as parking lots. A value of $S P=40$ was selected for Level 1 through iterative and interactive control. Many OOAbased studies continue to use a trial and error approach to determine the appropriate $S P$ as no universally accepted tool to determine the optimal scale exists (Aguilar et al. 2013, Hu and Weng 2010). The SP value is chosen based on a qualitative analysis (visual inspection) of the resulting segmentation and purpose of classification. The ESP tool proposed by Dragut et al. (2010) does provide a range of optimal scale parameters. The problem again is to choose the right $S P$ according to user requirements.

Our study deals with two scales of analysis, the object and settlement level. Since ESP tool uses only one image layer as input for optimal scale determination, its implementation in the current study was not useful. After experimenting with various values ( 15 to 60 with an increment of 5 for Level 1 and 150 to 500 with an increment of 50 for Level 2), we chose the appropriate $S P$ values for segmentation at the two levels. At Level 2, the segmentation was done at a coarser level to represent the settlement level of the ontology. The purpose was to delineate approximate boundaries of settlement primitives in slum areas. By settlement primitives, we mean objects representing an entire slum settlement or significant parts of a slum. Along with the three bands of the image, the GLCM contrast for the blue band (GLCMCon(B)) calculated using Haralick's method, was used as an additional layer of texture for this segmentation (Trimble 2014).

eCognition developer, the software used for OOA in this study, can create a temporary layer that can be used for segmentation or classification. A temporary GLCM layer was generated where values were calculated for each object individually at Level 1, using a 256 by 256 matrix, with a direction equal to $0^{\circ}$. This layer and the blue, green and red bands were used as input for segmentation at Level 2. A value of $S P=300$ was found visually satisfying to generate segments comprising areas with homogenous texture. Again, a value of 0.5 was assigned to $W$ shape to give equal weight to shape and spectral reflectance. The classes used for the higher level segmentation were built-up, vegetation and shadow. Most slums in Pune comprise highly dense buildings, and a low proportion of vegetation and open space (Table 4-1). Thus, slum areas have a low variance within segments and a clear contrast to planned 
areas. The use of a GLCM contrast layer helped to delineate segments representing the boundaries of slums as opposed to segments in planned areas that have a composition of different land cover features (e.g. buildings, vegetation, roads) (Figure 4-4).

Table 4-2 Definitions of the parameters used in the classification. For details, refer to Trimble (2014).

\begin{tabular}{|c|c|c|}
\hline Parameter & Description & Definition \\
\hline GR & Green ratio & $\begin{array}{l}\text { An index to measure vegetation, defined as } \\
(\text { green } /(\text { red }+ \text { green }+ \text { blue }))\end{array}$ \\
\hline Brightness & Brightness of object & $\begin{array}{l}\text { The mean intensity of all image bands for an } \\
\text { image object. }\end{array}$ \\
\hline GLCM & $\begin{array}{l}\text { Grey Level co-occurrence } \\
\text { matrix }\end{array}$ & $\begin{array}{l}\text { Proximal combinations of pixel brightness } \\
\text { values (grey Levels) within a particular band } \\
\text { of an image. }\end{array}$ \\
\hline $\mathrm{GLCM}_{E n t}(\mathrm{R})$ & $\begin{array}{l}\text { GLCM entropy for red } \\
\text { band }\end{array}$ & $\begin{array}{l}\text { The measure of orderliness within the red } \\
\text { band of the image and relates to textural } \\
\text { homogeneity. }\end{array}$ \\
\hline $\mathrm{GLCM}_{\mathrm{Con}}(\mathrm{B})$ & $\begin{array}{l}\text { GLCM contrast for the } \\
\text { blue band }\end{array}$ & $\begin{array}{l}\text { The amount of local variation within the blue } \\
\text { band of the image. }\end{array}$ \\
\hline Mode(R) & Mode for red band & $\begin{array}{l}\text { Returns the most frequently occurring pixel } \\
\text { value per object in the red band. }\end{array}$ \\
\hline Area & Area of object & $\begin{array}{l}\text { The number of pixels forming an image } \\
\text { object. }\end{array}$ \\
\hline Merge & Merge region & $\begin{array}{l}\text { Neighboring image objects of the same class } \\
\text { are merged. }\end{array}$ \\
\hline $\mathrm{RA}(\mathrm{S})$ & $\begin{array}{l}\text { Relative area of neighbor } \\
\text { object (shadow) }\end{array}$ & $\begin{array}{l}\text { The area covered by image objects of a } \\
\text { selected class, found around the selected } \\
\text { image object, divided by the total area of } \\
\text { image objects inside this area. }\end{array}$ \\
\hline MG & Mean green & $\begin{array}{l}\text { The mean intensity of all pixels forming an } \\
\text { image object in the green band. }\end{array}$ \\
\hline $\mathrm{D}(\mathrm{SL})$ & Distance to slums & $\begin{array}{l}\text { The distance ( in pixels) of an image object } \\
\text { to the objects of selected class. }\end{array}$ \\
\hline ES & Enclosedness by slums & $\begin{array}{l}\text { Image objects that are completely enclosed } \\
\text { by objects belonging to selected class. }\end{array}$ \\
\hline
\end{tabular}




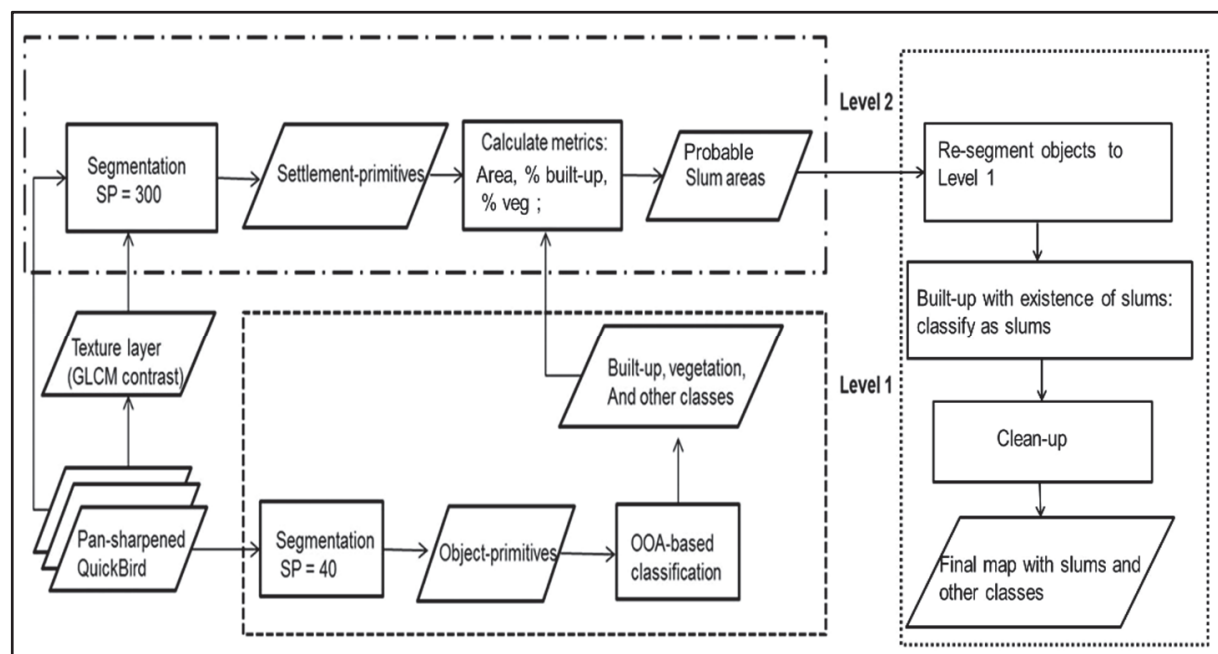

Figure 4-3 Method linking texture layer and spatial metrics to GSO at object and settlement level.

\subsubsection{Classification at Level 1}

Segmentation was followed by classification at Levels 1 and 2 respectively. Segmentation results in large number of image objects for which a variety of object characteristics or features can be calculated and subsequently used for classification. For each object, feature values such as layer mean values, shape characteristics, texture, size and contextual relations are computed based on purpose of classification (Benz et al. 2004). Rule-based classification, also known as membership function classifier, can be used to assign image objects to the desired class by integrating prior knowledge (Baltsavias 2004, Belgiu et al. 2014a). In this study, knowledge of slums in the form of local ontology was used as a basis to determine the most relevant features and corresponding thresholds to classify the image objects. A sequence of steps defining the class membership conditions forms a ruleset where each step comprises of a condition/rule leading to classification of a respective class. Several steps may be required to classify a single class. All these steps were executed in eCognition developer and are explained in detail below.

Classification of the segmented image was also performed in two steps. First, the non-built-up classes such as water, road, shadows and vegetation were classified. Due to the absence of the near infra-red (NIR) band, vegetation was classified using the customized arithmetic ratio, the green ratio (GR) (Table 42). GR values above 0.42 were used to classify vegetation. Shadows of trees and buildings were detected using the low values of brightness, and were classified using mean Brightness $<310$. These classes were useful in defining contextual relationships for characterization of built-up and also to avoid misclassifications. The vector layer of water bodies was used to classify water. 
Road classification in urban areas is quite challenging because the shadows of buildings and vegetation interferes with correct delineation. Vegetation, can in some cases, hide roads completely. Thus, the digitized road layer was used to classify roads. This road layer comprised of the centrelines of the roads; the objects overlapping the layer were classified as roads.

For the classification of built-up areas, spectral measures and texture were used. The spectral feature, mode for the red band, Mode(R), was first used to classify built-up. This feature calculates and returns the most frequently occurring pixel value in the red band in an object (Table 4-2). Mode(R) values above 360 corresponded to the built-up class, especially the bright-roofed buildings. The remaining built-up, comprising of mostly dark-roofed/old buildings, was classified using texture. Previous studies have shown the usefulness of texture for built-up classification (Belgiu et al. 2014a, Kohli et al. 2013). GLCM entropy of the red band was used to discriminate the remaining built-up from other land cover features. The entropy as a measure of disorder reflects the distribution of grey values and relates to textural homogeneity. By visual inspection, high values of entropy for the red band were found useful for classifying built-up. In this study, $\operatorname{GLCM}_{E n t}(\mathrm{R})>3.8$ was used to classify the remaining built-up area. Slightly lower GLCM $\operatorname{Ent}_{(}(\mathrm{R})$ corresponds to bright roofs and bare soil. Use of $\operatorname{Mode}(R)$ was helpful to avoid false positives from bare soil. The results of Level 1 were further used for classification of slums at Level 2.

\subsubsection{Classification at Level 2}

From the local ontology, it is evident that the slums in Pune are highly dense and often have a clear contrast to the formal areas (Figure 4-4). We used the Level 1 (object level) classification results to calculate the spatial metrics for the V-I-S for Level 2 settlement primitives. The built-up class, in this study, is synonymous to the impervious class as it includes building roofs and other impervious materials. Considering this particular urban context, the use of soil was insignificant for classification. Area, in terms of size of segments, is useful in identifying and characterizing slums (Kohli et al. 2013, Kuffer et al. 2014). Large Level 2 segments (value of Area > 14000 pixels) tend to correspond to slums and included the smallest slum in the study area. Out of these large segments those comprising of relative area of built-up $>50 \%$ and vegetation $<30 \%$ (Table 4-1) were classified as slum settlements. Subsequent steps involved bringing Level 2 classification result to Level 1 to get a final classified map together with other classes (Figure 4-6). Additionally a clean-up process was followed to eliminate false-positives from the classified slums and reclassify any observed, misclassified slums. These steps are explained in the following section. 


\subsubsection{Clean-up}

The Level 2 segments (classified and unclassified) were converted to objects at Level 1 (Figure 4-3). This step re-segmented the larger segments at Level 2 to smaller Level 1 segments. The built-up objects at Level 1 , which overlapped with slum segments from Level 2, were re-classified as slum. The feature, merge region, was used to merge neighboring objects of the slum class. A threshold of Area $\leq 14000$ pixels was used to remove small buildings classified as slum. Relation to neighboring objects was used to remove falsepositives from the Old City (Table 4-2). By visual inspection, the value of relative area of neighbor object shadow, $\mathrm{RA}(\mathrm{S})>0.24$ was found appropriate to remove buildings from Old City classified as slums. Further, mean green, MG > 540 was used to remove false positives that occur at the bottom-right of the scene, displaying higher values as compared to slum (Figure 4-1). These were large buildings that display low contrast and thus classified as slum. To include the misclassified objects on the fringes of slum areas, distance to slums, $D(S L)<1$ pixel was used. After merging the classified objects of the same class, the feature enclosed by slum (ES) was used to include any misclassified object embedded within a slum area. Table 4-3 shows the final ruleset summarizing the sequence of steps followed for classification.

\subsubsection{Accuracy assessment}

Random stratified sampling was used to assess the accuracy at Level 1. A total of 250 points were generated with the aim of having a minimum of 50 points for each class (Herold et al. 2008, Scepan 1999). A standard confusion matrix was used to calculate the overall accuracy, user's and producer's accuracy (Congalton 1991). The points were visually interpreted on the Quickbird image for reference.

At Level 2, the slum layer provided by MASHAL was used (Figure 4-2). The accuracy of slum classification was assessed by using the "error matrix based on training and test area (TTA) mask" in Ecognition developer (Trimble 2014). The TTA mask when used to calculate the accuracy of a single class, measures the matching of reference pixels and classified pixels inside the reference areas (Hofmann et al. 2008). The error matrix results, thus, gives the percentage of agreement between the reference map and an error of omission respectively. 
Table 4-3 Ruleset summary and the quantitative classification criteria with the sequence from top to bottom.

Seq.

\section{No. Classification Steps Ruleset}

Generate object primitives at Multiresolution segmentation Level 1

$\left(S P=40, w_{\text {shape }}=0.5, w_{\text {compt }}=0.5\right)$

2 Classify Roads and Water

Assign class using thematic layer

3 Classify Vegetation

$\mathrm{GR} \geq 0.42$

4 Classify Shadows

Brightness $<310$

5 Classify Built-up

$\operatorname{Mode}(R)>360, G L C M_{\text {Ent }}(R)>3.8$

6 Generate settlement primitives at Level 2

Multiresolution segmentation

$\left(S P=300, w_{\text {shape }}=0.5, w_{\text {compt }}=0.5\right)$

$7 \quad$ Classify Slums

Area $\geq 14,000$, built-up $>50 \%$ and veg $<30 \%$

$8 \quad$ Clean-up

Area $\leq 14000, \mathrm{RA}(\mathrm{S}) \geq 0.24, \mathrm{MG}>540, \mathrm{D}(\mathrm{SL}) \leq 1$, ES

\subsection{Results}

\subsubsection{Segmentation}

For segmentation at Level 1 , a value of $S P=40$ worked well to segment builtup, vegetation and shadow appropriately. For the built-up class, the segments represent individual buildings in the non-slum areas and clumps of buildings in slum areas (Figure 4-5), as the slum buildings tend to be small and densely packed, making the individual structures indistinguishable at $60 \mathrm{~cm}$ resolution of the Quickbird image. For segmentation at Level 2 we used a value of $S P=$ 300 to segment settlement-primitives (Table 4-3). The use of temporary layer of GLCMcon(B) in addition to blue, green and red bands resulted in segments with homogeneous texture representing slum settlements (Figure 4-4). Segments in the planned areas thus comprise of a mix of buildings, vegetation, shadows and other classes. 


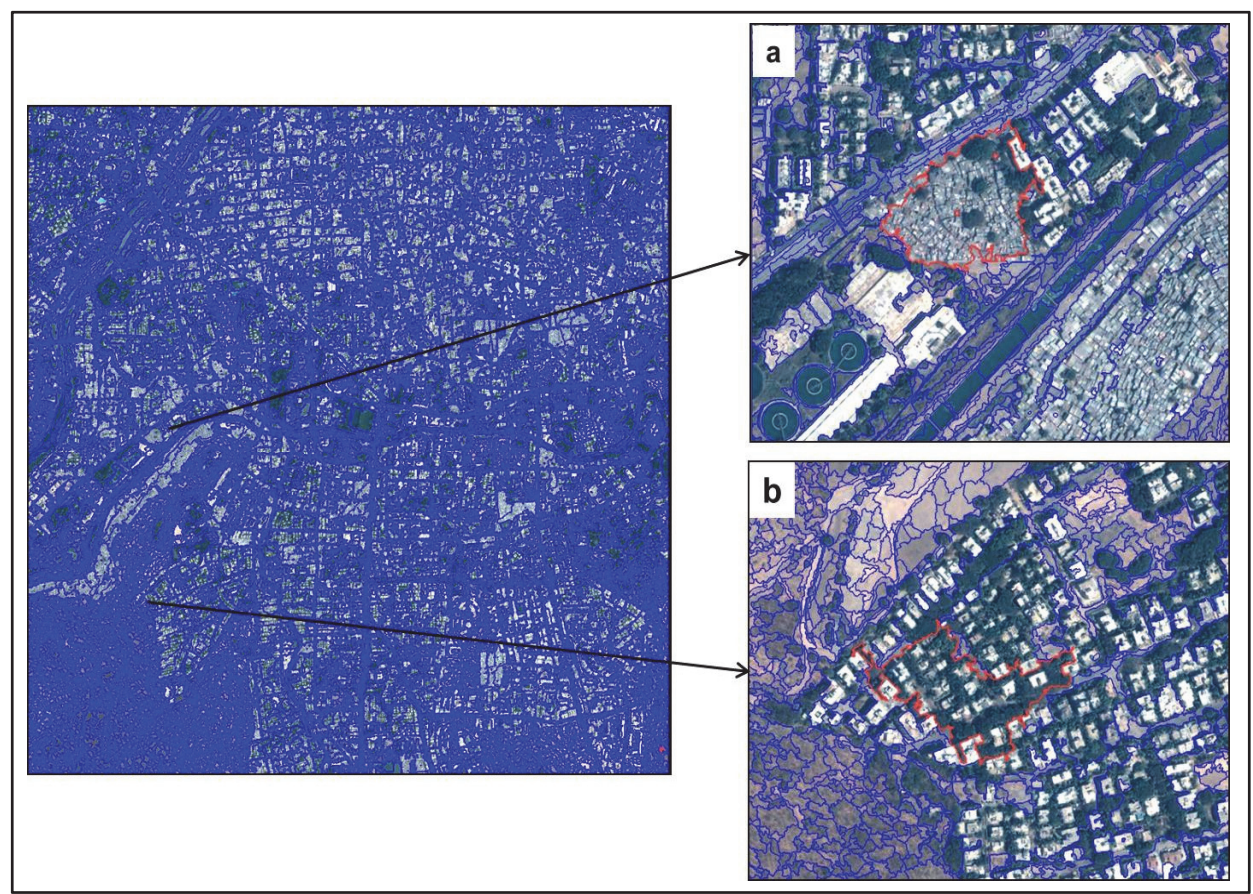

Figure 4-4 Settlement-primitives at Level 2 using texture layer GLCM contrast: (a) Highly dense buildings in slums outlined and displaying a clear contrast to neighbouring segments; (b) Planned area with segments comprising different land cover features.

\subsubsection{Classification at Level 1}

Figure 4-5 shows the classified built-up, vegetation and other classes at Level 1. The segmented individual buildings in the planned areas and clumped buildings in slum areas were classified as built-up. Some confusion between the shadow and vegetation classes was observed, specifically with the shadow of tall buildings being classified as vegetation. Due to the use of GR for vegetation classification, such misclassifications occurred, though they did not directly affect the classification of slums at Level 2 as slum buildings hardly display any shadows due to their compactness. Other classes, water and roads were classified appropriately using digitized vector layers. These classification results were used as a basis for the Level 2 classification. 


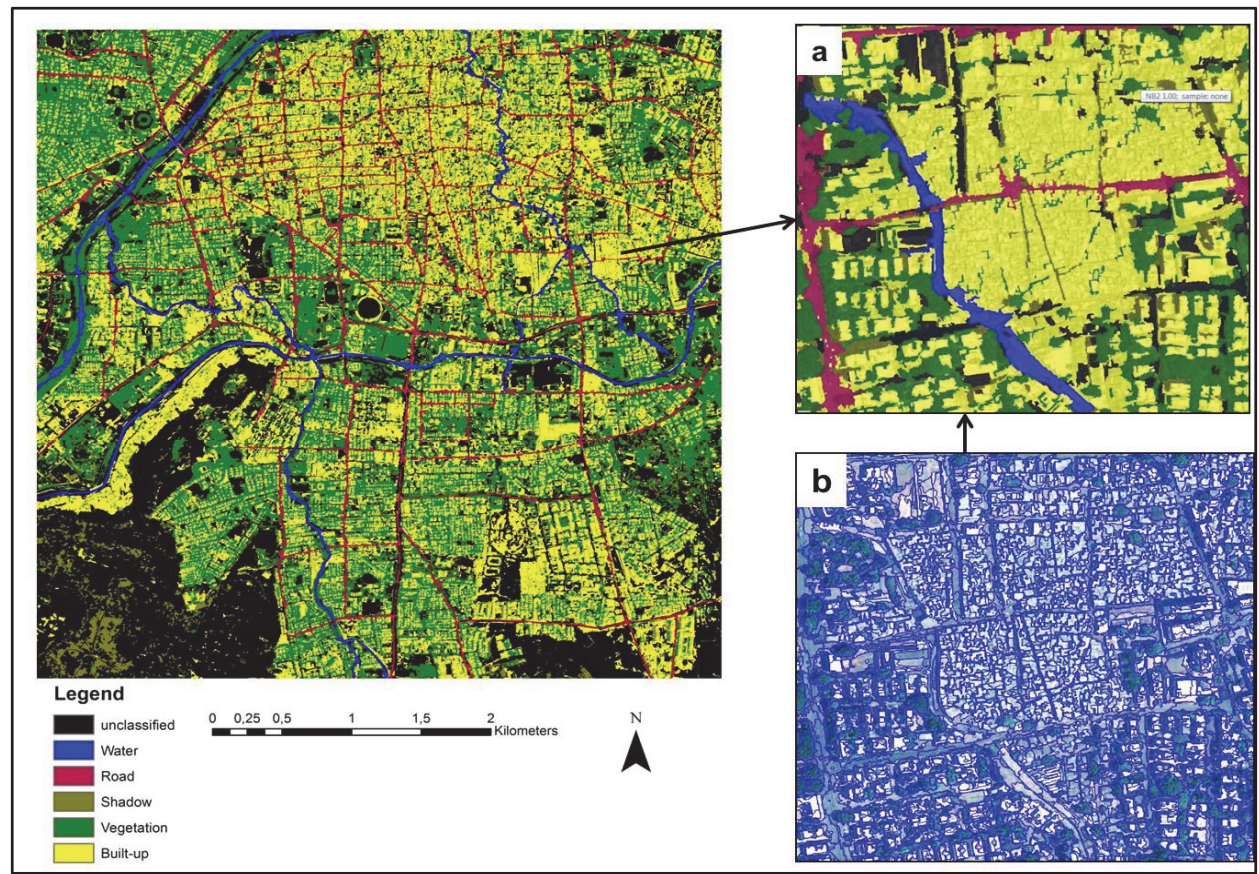

Figure 4-5 Classification of image at Level 1: (a) Magnified view of built-up classified in slum area; (b) Segmentation result of the same area.

\subsubsection{Classification of slums}

The segments representing settlement-primitives of slum areas were mostly comprised of compact buildings (Table 4-1). Large segments comprising builtup $>50 \%$ and vegetation $<30 \%$ (Table $4-1$ ) were classified as slum areas. The proportion of built-up may be as high as $70 \%$ for entire slum settlements. A lower value was used, however, to include parts of slums with lower built-up density. Slums with high contrast to their surrounding areas were successfully delineated. False-positives existed in the Old City area that also comprises areas of dense, often old and dilapidated buildings. The Old City is comprised of many multi-storied buildings displaying a clear shadow. Relation with shadow, RA(S) was used to remove these false-positives. Misclassifications were also found in other areas where comparatively smaller segments have high built-up proportions such as the market areas. Finally, a sequence of steps using MG, D(SL) and ES was used for clean-up (Table 4-3). Figure 4-6 shows the classification results and the ground pictures of the slums and part of the Old City. 


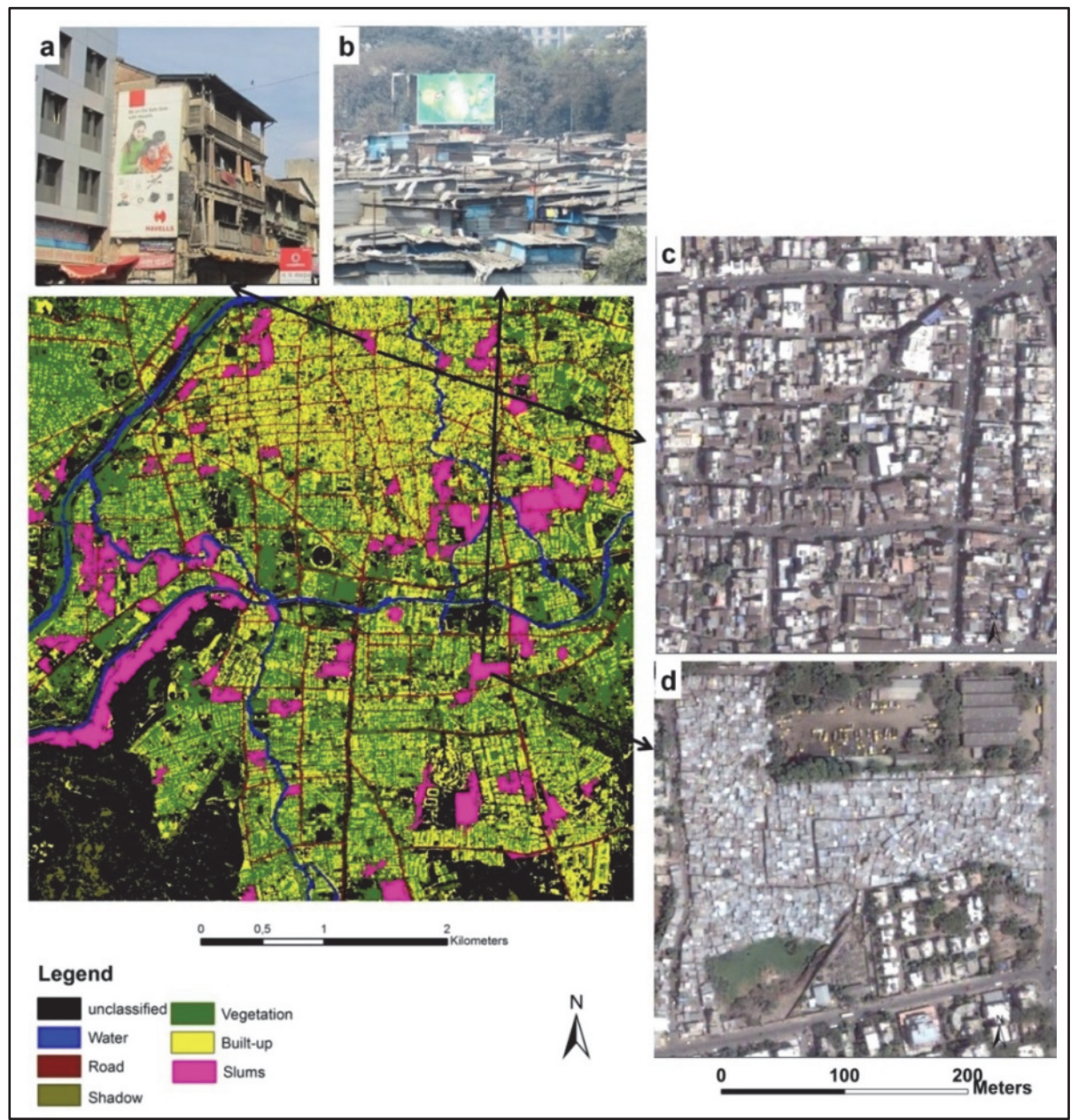

Figure 4-6 Final classified map of the study area with slums, ground pictures and corresponding zoomed views of image: (a) Ground photograph of buildings in Old City; (b) Ground photograph of a typical slum settlement; (c) Magnified view of a part of Old city misclassified as slum on final map; (d) A typical slum settlement.

\subsubsection{Accuracy assessment}

After classifying the whole scene (Figure 4-6), the accuracy assessment was carried out.

\subsubsection{Accuracy of basic land cover features}

The Level 1 classes were separated using spectral information and texture. The overall accuracy of the Level 1 classification was $81 \%$ (Table 4-4). The classes assessed were shadow, vegetation and built-up. The 'others' class (shown as unclassified in map) comprise of the remaining land covers in the scene such as bare soil and barren land. These classes were not directly relevant for this 
study, thus left as unclassified. The relevance, though, is in terms of misclassifications. For example, built-up was classified with maximum accuracy with high producer accuracy ( $88.24 \%)$ as well as high user accuracy (82.19 $\%$ ) though some confusion with bare soil (others) is evident. Comparatively, vegetation has lower producer accuracy $(80.88 \%)$ due to confusion with the shadow class. Vegetation has false-positives from the shadow class and viceversa. Shadow is classified with lowest accuracies (62\% and $75.61 \%)$. This is due to spectral similarity to barren land, dark colored roofs and vegetation. The goal of Level 1 classification was to provide a land cover product which could form the basis for further analysis at Level 2. An accuracy of $81 \%$ can be considered good and encouraging for Level 2 analysis (Herold et al. 2003).

Table 4-4 The producer's and user's accuracies of the land cover classes at Level 1; PA $=$ producer accuracy, UA = user accuracy, OA = overall accuracy and Kappa = Kappa agreement index.

\begin{tabular}{lllllllr} 
& Shadow & Vegetation & Built-up & Others & Total & PA (\%) & UA (\%) \\
\hline Shadow & 31 & 10 & 0 & 0 & 41 & 62.00 & 75.61 \\
Vegetation & 10 & 55 & 0 & 0 & 65 & 80.88 & 84.62 \\
Built-up & 4 & 1 & 60 & 8 & 73 & 88.24 & 82.19 \\
Others & 5 & 2 & 8 & 56 & 71 & 87.50 & 78.87 \\
Total & 50 & 68 & 68 & 64 & OA & $\begin{array}{l}80.80 \% \\
0.74\end{array}$ \\
& & & & & Kappa & \\
\hline
\end{tabular}

\subsubsection{Accuracy of classified slums}

The accuracy results demonstrate that $60 \%$ of the pixels of the reference map were also detected as slums in classification at Level 2, i.e. the error of omission was $40 \%$. Errors in the classified map can be attributed to a number of reasons. First, the amount of generalization in the outlines of the reference layer could have led to errors (Figure 4-7a). Secondly, the process of defining slums in India can be rather complicated due to political and administrative interests. The definition used by MASHAL for slum delineations, which incorporates these political and administrative perspectives, also impacts our results. Thus, many areas that are morphologically slum-like are classified as slums but are absent in the reference layer (Figure 4-7b). However, in a data poor environment like India, this is the best reference that could be procured. Thirdly, some of the polygons of the reference layer show vegetation on ground instead of slum areas (Figure 4-7c). These could be small slum settlements hidden under trees and hence, missed out in classification. During field work, we observed that many buildings in the Old City and market areas were as dilapidated as slum buildings, but these areas are, by definition, not considered slums by MASHAL but some of these areas are classified as slums. High density built-up and low vegetation are used as a proxy for slum presence. Though this 
approach has worked for classifying slums in the scene, there are misclassifications from high density non-slum areas (such as the Old City and market areas). These misclassifications could also be attributed to the errors stemming from basic land cover classification (Figure 4-7d). There are some parts of the Old City where use of shadow could not remove misclassified slums. Though some of the misclassifications were removed by using MG, false-positives still exist. Finally, the use of a single class for error matrix calculation results in the percentage of agreement between the reference map and the slum classification. This is because of the absence of non-desired class in the reference layer (non-slum in this case). The overall accuracy thus ignores the false positives outside the reference layer and also the unclassified pixels inside the reference layer.

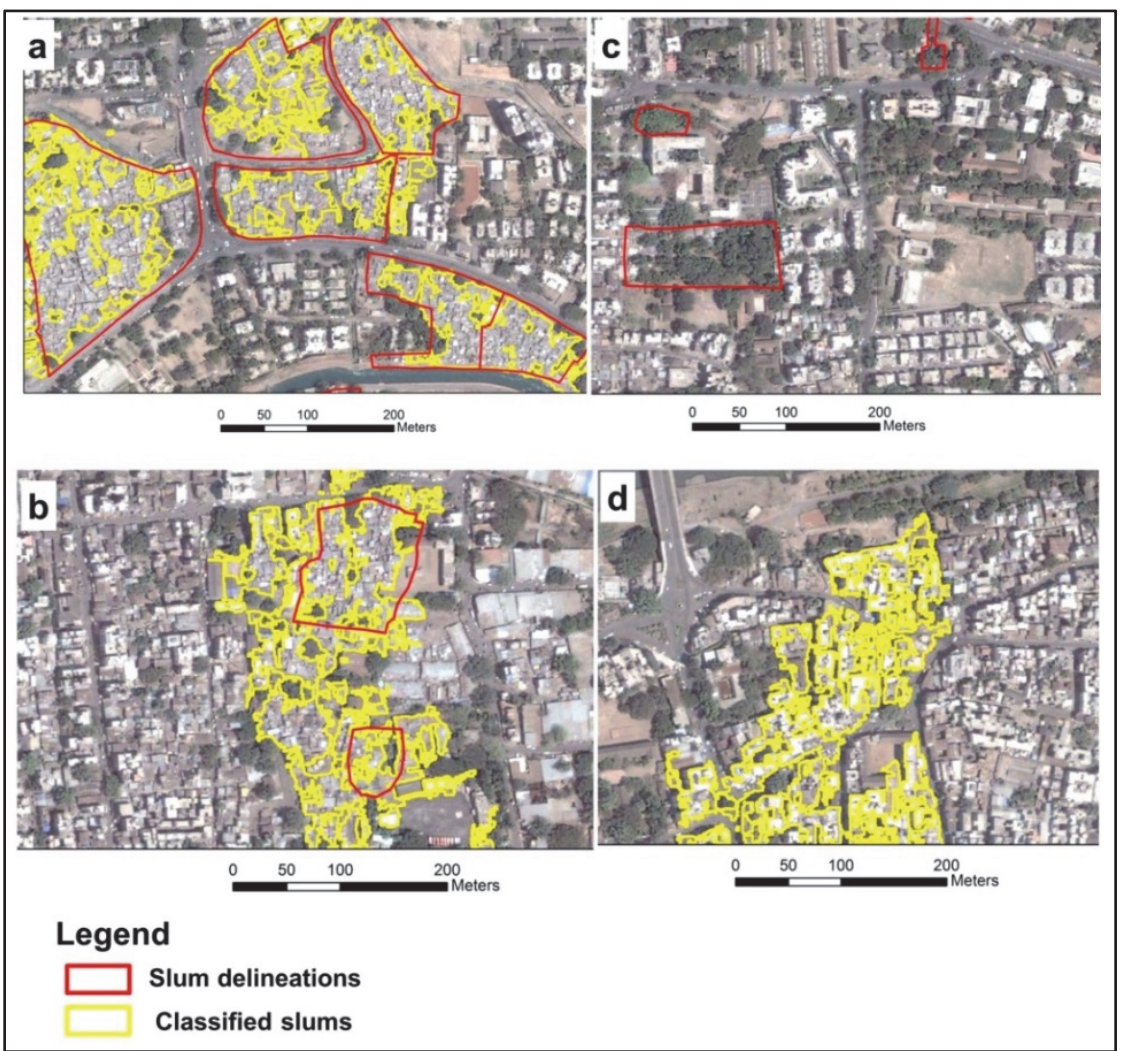

Figure 4-7 Figure showing source of error in classification resulting from: (a) generalization; (b) political/administrative definition; (c) vegetation cover; (b) falsepositives from Old City. 


\subsection{Discussion}

Unprecedented urbanization in many countries of developing world has led to growth of slums. Slums provide an affordable housing option to those who cannot find a place in the formal housing market. Slums form an integral part of city landscapes in many cities of India. With the growing economy, more and more people opt to leave villages for cities in search of better opportunities. Pune city in India is one of the fastest growing metropolitan with almost 40 percent of its people living in slums (MASHAL 2011). Considering the scale of slum-population, mapping existing slums may facilitate better policy formulations for improvement and to provide infrastructure or alternatives. It could also be important to monitor such developments to get updated information for crisis management (PMC 2012). The fact that many slums in Pune are located on ecologically sensitive areas, considered unattractive for any planned development, substantiates this need. Mapped slum settlements may be used as an important input for climate change mitigation activities (Kit et al. 2012). Considering the location of a settlement (environs) in addition to other indicators, in a previous work, a GSO was developed to be used as a basis for systematic slum identification using VHR image (Kohli et al. 2012). Subsequently, the GSO was adapted for slum detection and classification in the Indian city of Ahmedabad (Kohli et al. 2013).

Developing rulesets in OOA requires considerable image analysis skills, expertise in the application area and time (Belgiu et al. 2014a). Thus, there have been recent efforts to make rulesets/methods more transferable for wider application (Kohli et al. 2013, Hofmann et al. 2011, Belgiu et al. 2014a). In our previous work, we identified set of stable parameters, in an OOA environment, that worked on subsets of varying characteristics for classifying built-up and slums. In this study, we take that work further by developing a method that combines the GSO, parameterization in OOA (Table 4-1) and V-I$S$ model (Ridd 1995) to detect slums in Pune city. This approach not only considerably reduces the number of parameters used in the ruleset, it also presents the possibility of successful application on similar morphogical context. Our study also builds upon similar work where the authors use ontology-driven class definitions for classification of informal settlements in Quickbird image of Rio de Janiero (Hofmann et al. 2008). The complexity of ruleset in that study, however, may restrict the transferability of the method.

The V-I-S model used in our method helped to capture the characteristics of slums at settlement level of the local slum ontology. Abundance of builtup/impervious and low vegetation served as a proxy to the presence of slums. A hierarchical approach comprising the two levels of ontology, the object level and settlement level, was used in an OOA environment. The object level represented the basic land cover features of the V-I-S model. The parameters 
GR, Brightness, mode $(\mathrm{R})$ and $\mathrm{GLCM}_{E n t}(\mathrm{R})$ were useful for basic land cover classification of vegetation, shadow and built-up, respectively. Roads and water were classified by using vector layers. Roads classification is one of the trickiest one in RS as vegetation and shadow can hide them completely. With RS offering a bird's eye view, we are left with conceptual problems where an object virtually has two classes, vegetation and road. The choice, then, could be driven by purpose of classification. To overcome this problem, we digitized the major/main roads in the study area. The minor/secondary roads were purposely left. The classification of roads in planned areas could negatively impact quantification of vegetation class. This could further influence our results as vegetation and built-up are used as a proxy for slum presence.

The overall accuracy achieved for land cover classification is $81 \%$ with the Kappa agreement of 0.74 . Whereas there have been discussions in previous research on the suitability of error matrix and kappa for measuring accuracy (Pontius and Millones 2011), there are many OOA studies that continue to use them (Belgiu et al. 2014a, Belgiu and Drăgut 2014, Herold et al. 2003, Myint et al. 2013). In the absence of a detailed and updated reference map, a set of randomly selected pixels was considered to be representative of the ground condition (Myint et al. 2013, Congalton 1991). There are mis-classifications where built-up mixes with bare soil, specifically dark-colored roofs made of rusted corrugated iron sheets display low $\operatorname{GLCM}_{E n t}(\mathrm{R})$ similar to rough and porous bare-soil.

There is noticeable mixing between the shadow and vegetation class with shadow being classified with minimum accuracy. Due to the sun angle at the time of acquisition of the image, some smaller trees seem to hide under the shadow of higher trees with large canopies; resulting in low brightness values and hence, being classified as shadows. The use of a near Infrared (NIR) band would have allowed a better discrimination of vegetation from other classes. The purpose of our study to use only RGB data is to show what can be achieved when NIR data are not available. This is a common situation in many cities that, if using satellite images at all, restrict themselves in purchasing RGB data because they are primarily interested in the image as a backdrop or for data extraction via interpretation. In addition, many cities do not have sufficient inhouse remote sensing knowledge to fully understand the analytical implications of having access to all bands. As RGB data are commonly available within city authorities it is relevant to examine whether OOA approaches in combination of reasonably simple V-I-S model can also generate acceptable results (slum maps in this case) as this would mean that the approach is also widely applicable.

The settlement level provided boundaries for the quantification of land cover features. Automatic delineation of quantifiable segments for appropriate spatial 
metrics analyses is challenging considering the heterogeneity of urban areas. Previous studies (Herold et al. 2003, Herold et al. 2002) used manually delineated homogeneous urban regions, also referred to as Homogeneous urban patches (HUP), or enumeration units (Weeks et al. 2007) for determining land use using spatial metrics analyses. A recent publication automated generation of HUPS at settlement level by using large $S P$ value for segmentation (Kuffer et al. 2014). We used the same approach by using a $S P$ $=300$ value to generate settlement-primitives representing parts of slum settlements and mix of different land cover features in planned areas. We do refrain from terming settlement-primitives as HUPs as they do not entirely comply with the 'rules for HUPs' presented in Herold et al. (2002). In addition to the three bands of the Quickbird image, we used the texture GLCMcon(B) to segment settlement primitives. $\operatorname{GLCM}_{\mathrm{con}}(\mathrm{B})$ have been identified as a stable parameter for slum classification (Kohli et al. 2013). A large value of SP helps to generate large segments representing slum settlements, but it also poses a challenge as under-segmentation may occur. This is likely to be the case if non-slum buildings on the fringes are included in a segmented slum due to spectral similarity. Use of texture largely solved this problem by restricting the segmentation as most slums in Pune comprise highly compact buildings and hence low variance within segments (Figure 4-4). Subsequently, Area, expressed in terms of number of pixels and spatial metrics, was used to classify slum settlements. The parameters Area, RA(S), and MG were suitable for eliminating false positives. $\mathrm{D}(\mathrm{SL})$ and $\mathrm{ES}$ were uniformly useful for reclassifying any misclassified slum objects embedded within the slums.

The accuracy value of $60 \%$ is encouraging considering the complexities involved in image-based slum identification (Kuffer et al. 2014, Kohli et al. 2013). Low accuracy can be attributed to a number of reasons as listed in the accuracy section. The use of this reasonably simple approach shows the potential for transferability of our method. Care must be taken, however, when this method is applied on a context with completely different characteristics. A suitable approach would be adapting the GSO to that particular context and modifying thresholds or parameters based on its characteristics. Addition of new parameters may also be required for improved classification. It is interesting, that some of the parameters such as $\operatorname{GLCM}_{E n t}(\mathrm{R}), \mathrm{GLCM}_{\text {con }}(\mathrm{B})$, Area and ES identified as most stable for built-up and slum classification in a previous study in another Indian city, Ahmedabad (Kohli et al. 2013), could be used in this study as well.

Previous work has also been done on two small extracts of the city of Sao Paulo where the knowledge-based system Inter-Image was applied (Novack and Kux 2010). For such small areas, higher accuracies were obtained, as upon a careful selection these have a higher uniformity: the dwellings were raised in approximately the same period and have developed along similar lines. In 
terms of the GSO, the Sao Paulo study is at the object level (Level 1). Our study builds upon this work by providing a methodology to consider a larger area that contains more variation in history and ground conditions. This unavoidably leads to a decrease in accuracy. A possible way to overcome this reduction would be to use a hierarchical approach: splitting up the larger area into a mosaic of smaller sub-areas, classifying these and then integrating towards the settlement level. We leave this for future work.

Slums are not just physical entities; they also have administrative, political and local perceptions attached to them. Thus, it can be difficult to identify them solely by visual interpretation, particularly if one lacks local knowledge. In the process of developing a semi-automated slum identification process, we found that ontologies can help to bridge the gap between technology and real world by integrating that expert (local) knowledge about the context. It also helped to systematize the process by considering different spatial levels where a set of indicators may be chosen depending on context for an OOA. In Pune, slums generally have a distinct appearance compared to planned residential complexes but there were a few exceptions e.g. the Old City core which exhibited similar characteristics as slums. Pune and many other cities in India have a historic old centre or core. If these city-cores have dilapidated over time; they are likely to exhibit similar physical characteristics as many slums and can therefore be readily misclassified as such (Figure 4-6). During our field-visit to Pune, we observed that some of these buildings had already collapsed and many need upgrading in order to be considered as durable houses. Though shadow was useful in removing false-positives from the Old City, the inclusion of some buildings negatively impacts our classification, as these are excluded from the reference layer that is based upon a different political-administrative definition of slum (Figure 4-7). This problem exists in Pune and is known to also exist in other Indian cities. It is also quite conceivable that this issue will arise in other contexts as well.

Additional information in terms of surface and terrain elevation conditions may help in improving the results: many slums are located on steep slopes and surface height information can be used to differentiate slum buildings from formal, high-rise buildings. Pune's slum buildings generally tend to be made of less-durable materials and thus have lower heights. Other than the false positives in the Old City the methodology works well in the other parts of the image, especially for highly dense slums showing a clear contrast to other builtup areas. Though there are false-positives from market areas because of their relatively large and highly dense roofs contributing to the high roof coverage. The larger slums with a relatively uniform texture are generally identified correctly, while some small slums are often missed because at settlement level they merge with other land cover features. 
Finally, the low agreement between the reference and slum classification can also be attributed to the uncertainties in the reference data itself. The reference data is prepared by manual image interpretation, which may contain uncertainty and hence negatively impact accuracy results (Figure 4-7). The expertize of the interpreter in this field, RS skills and the time invested may have led to discrepancies in interpretation (Van Coillie et al. 2014). In case of slums, different conceptualization, fuzzy boundaries, poor definitions and variability could add to uncertainties in delineations. Inaccuracies in classification can thus be due to errors in the reference data (Albrecht et al. 2010) and should be understood. This forms the topic for our ongoing investigation where we are studying the uncertainties attached with the existence/presence and spatial extent of slums.

\subsection{Conclusions}

This study presented a method for slum detection from VHR images based on the morphology of the built environment as expressed in generic slum ontology (GSO). It consists of segmentation followed by hierarchical classification using an object-oriented image analysis (OOA). It used texture in combination with spatial metrics based on the V-I-S model and was applied to a Quickbird image of the city of Pune, India. It turned out to be a promising method for slum identification. Contextual knowledge in the form of a local slum ontology guided the classification process. Our analysis identified that textural features derived from a grey level co-occurrence matrix (GLCM), contrast (GLCMcon) in addition to three bands of an image were appropriate for segmentation at settlement level. A clear difference in classification accuracy was revealed between the two ontological levels.

The overall accuracy at Level 1 of $80.8 \%$ was remarkably high and adequate as an input to the GSO considering the limited spectral resolution of the Quickbird image and the absence of near infrared (NIR) band. The percentage of agreement between the reference layer and the slum classification at the settlement level was $60 \%$. This value is promising considering that a rather simple method was applied for slum identification, consisting of the size of image segments (Area), proportions of built-up and vegetation. The parameters: relative area of shadow $R A(S)$, mean green (MG), distance to classified slums $D(S L)$ and enclosed by slums (ES) were useful for the final classification. Our results are potentially useful for city managers especially in cities where no alternative data is available to get updates regarding slums on the ground. Further study of characteristics of slums in terms of patterns, size, density and their manifestations in the images may be useful. In such future research, relations with other classes, derived at the environs level of ontology are likely to be used to refine results. 


\title{
Chapter 5
}

\section{Uncertainty analysis for image interpretations of urban slums*}

\author{
"There is no such uncertainty as a sure \\ thing" \\ - Rabert Burns
}

* This chapter is based on the article:

Kohli, D., Stein, A., Sliuzas, R. 2013. Uncertainty analysis for image interpretations of urban slums (Submitted to Journal of Computers, Environment and Urban Systems). 


\begin{abstract}
Image interpretations can be used as reference data for accuracy assessment in object-oriented image analysis (OOA). Such interpretations, however, also contain uncertainties which may negatively impact the accuracy. In this research, we study the spatial uncertainties related to the delineations of slums as observed from VHR images in the contexts of Ahmedabad (India), Nairobi (Kenya) and Cape Town (South Africa). Nineteen image interpretations and supplementary data were acquired for each context by means of semistructured questionnaires. Slum areas agreed upon by different experts was determined. Uncertainty was modelled using random sets, and boundary variation was quantified using the bootstrap method. Results show a highly significant difference between slum identification and delineation for the three contexts, whereas the level of experience in slum-related studies of experts is not significant. Factors of the built environment used by experts to distinguish slums from non-slum areas or leading to deviations in slum identification are discussed. We conclude that uncertainties in slum delineations from VHR images can be quantified successfully using modern spatial statistical methods.
\end{abstract}




\subsection{Introduction}

Slums are home to a growing proportion of urban dwellers in developing countries. Common methods for spatial data collection on slums are field-based and thus time consuming and difficult to update regularly. This may result in inadequate intervention by the concerned authorities and inability to monitor changes on ground. UN-HABITAT, in a number of publications, has substantiated the need of improved information on slums (UN-HABITAT 2010, UN-HABITAT 2014, UN-HABITAT 2003c). With enhanced knowledge and information, better policies can be formulated and strategies for future could be envisaged. The availability of Very High Resolution (VHR) satellite images in combination of Geographic Information System (GIS) exhibits a strong potential in identifying and characterizing slums. Using VHR images, remote sensing (RS)-based methods can help to have a city or country wide measure of slums and facilitate comparison on global scale. Several recent studies have shown the potential of using RS-based methods, specifically object-oriented image analysis (OOA) for mapping slums (Hofmann 2001, Hofmann et al. 2008, Kohli et al. 2013, Kit and Lüdeke 2013, Kit et al. 2012). As pointed out in most of these studies, however, slum identification and classification using $\mathrm{RS}$ is a complicated task.

Urban environments across the world are composed of a number of land cover features. Urban forms may not only vary amongst different countries but also within a country. Among many factors, these variations can be attributed to the historical origins, urban policies and financial wellbeing of a country. This poses a challenge in terms of developing a common classification technique to fit various contexts. Slums, being a component of such urban environments and geographic locations, exhibit different appearances and definitions. This interferes with developing a generic method for slum detection.

In our previous work (Kohli et al. 2012), we developed a generic slum ontology (GSO) which can be used as a basis for RS-based slum classification. The GSO is a framework comprising the morphological properties of the built environment which can be used to characterize slums. Its strength is contextadaptation by integrating expert knowledge. This leads to a knowledge base that can be used in turn to encode classification rules in OOA (Kohli et al. 2013). Creation of rules in OOA mimics the cognitive approach of visual image analysis. Classification using $O O A$ is based on conceptual understanding of features of interest and may differ if performed by different operators (Belgiu et al. 2014a). Image interpretation, thus, contains uncertainty which may negatively impact the classification results.

Further, to evaluate the results of OOA, accuracy assessment has to be carried out, e.g. by making a comparison with reference data. In many cases, a 
manual image interpretation is used as the reference. This approach has drawbacks in terms of the reliability of accuracy assessment results. The expertise of the interpreter in a particular field, RS skills and the time invested may lead to discrepancies in interpretation (Van Coillie et al. 2014). For slums, fuzzy boundaries, poor definitions, lack of local knowledge and variability may add to uncertainties in delineations. Inaccuracies in classification may therefore not be caused by classification alone, but may occur due to the uncertainty or errors in the reference data (Albrecht et al. 2010). If such data are used for assessing the accuracy of object based classification, it is first important to understand the attached uncertainties. OOA based methods are lately being used for classification of slums but negligible research has been done to address the uncertainties related to slums. Past research mostly presented a crisp classification method with a map of slum/non slum (Kit and Lüdeke 2013, Hofmann et al. 2008). Such a dichotomy may not always be applicable and requires further study in terms of uncertainty related to slum interpretation.

Several studies have evaluated the correspondence between image interpretations by different interpreters (Congalton and Mead 1983, Lunetta et al. 2001, Foody and Boyd 2013, Edwards and Lowell 1996). These studies focused on evaluating agreements of interpreters on different classifications and mostly focused on rural applications. In particular, interpreters asked to distinguish between different forest land-cover types, reported a wide variation. Studies of the classification of urban areas have been given less attention. Urban areas are generally considered as ensemble of objects with determined boundaries (Campari 1996). They comprise objects like buildings, roads and pavements that may be easily distinguishable from one another. Alternately, there are uncertain objects or concepts in an urban area, the accurate delineation of which can be complicated. An example is the delineation of the boundary of a city or classifying urban and suburban areas (Stein et al. 2009). Other examples may be distinctions between private and public open space, vegetated/landscaped areas in cities.

As stated by (Couclelis 1996), "Boundaries constitute the outer limits of individual entities, but also the locus where two or more different entities meet; they enclose and separate, divide and join, distinguish and juxtapose, contain and include, create interiors and exteriors, help tell same from other". Taking this definition, the current study considers uncertainty as the inability to draw a clear boundary about an object's existence, and where it ceases to exist. In a remote sensing environment, this links image features with objects on ground. Some boundaries could also be vague on the ground, as for example the boundary of a slum. Slums have different appearances and definitions depending on context, making it challenging to detect with RS techniques. This considers first the question whether a slum exists, and if so: how its extent can be determined. Related uncertainty is termed existential and extensional 
uncertainty, respectively(Molenaar 2000). Existential uncertainty expresses the uncertainty about the existence of a slum in reality. It thus refers to the possibility of existence of a slum as delineated by experts on an image and depends upon their inexperience and conceptual differences in interpretation. Extensional uncertainty implies that the area covered by a slum can be determined with limited certainty, i.e. with boundaries that reflect different perceptions of slums by experts. Extensional uncertainty in slum identification includes differences in expertise among the experts, the applied slum definitions, time invested and the degree of generalization.

The aim of this study is to study deviations in slum identification and their delineations as observed from VHR images. Existential and extensional uncertainties of slums are estimated using images from three contexts interpreted by 19 experts. Images from Ahmedabad (India), Nairobi (Kenya) and Cape Town (South Africa) were used. First, the percentage of slum area agreed by most experts and the uncertain area is calculated. Second, we model uncertainty in terms of random sets and apply bootstrap sampling methods to show confidence in various delineations and boundaries. Finally, we identify the factors of the built environment that experts use to distinguish slums from non-slum areas and also study the factors leading to deviations in slum identification in the three contexts.

\subsection{Method}

\subsubsection{Data collection}

In this study we address the existential and extensional uncertainty of slum identification by taking input from respondents on three different urban environments. We carried out a semi-structured survey for collecting data. By means of questionnaires, data on personal profile of respondents, expertise in remote sensing and their perception/definition/experience of slums was collected. In particular, the questionnaire consisted of questions on how respondents define slums (open-ended question), specific characteristics of slums in their respective context followed by a section on delineating slums (Figure 5-2). The responses were used to understand how respondents perceived a slum and in this way helped to comprehend their decision-making steps. The responses also provided insight into the variability and similarities of slums in various contexts. The results of this survey were primarily used to develop the GSO as documented and presented in Kohli et al. (2012).

\subsubsection{Data collection on image interpretation}

Respondents were asked to identify and delineate slums in satellite images of three cities acquired from Google Earth (Figure 5-2). For digitization of slums, . $\mathrm{kml}$ files linking to the snapshots of chosen Google Earth images were 
provided. Google Earth provides the possibility to digitize polygons on image snapshots and save them in . $\mathrm{kml}$ format. The respondents were asked to return the. $\mathrm{kml}$ files with the slum digitizations on the respective images and to list the factors they used for identification and delineation. Additionally, they were asked to mention the reasons, if any, for not being able to identify slums. To measure the confidence in the quality of their delineations, respondents rated their confidence on a five-point Likert scale, ranging from one (very poor) to five (excellent). For measuring the uncertainty, the three sets of 19 delineations were first converted from . $\mathrm{kml}$ to shapefile and combined using the union function in ArcGIS to form a single layer (Figure 5-4) that shows the agreement levels of slum delineations. The area identified by the maximum number of experts, compared to the total identified slum area was used to calculate the uncertain areas.

\subsubsection{Selection of respondents}

People having a combination of slum and RS knowledge were selected as respondents, also referred to as experts throughout this study. A total of 50 respondents participated in the survey. Out of these, 19 respondents returned the. $\mathrm{kml}$ files with the digitized slums which could be included in uncertainty analysis for image analysis. The invalid responses were incomplete or unattempted digitizations, scanned copies of delineations or the questionnaires returned as hard copies. Nonetheless, theoretical input from all 50 respondents was instrumental in the formation of the GSO as explained in Kohli et al.(2012). The survey was carried out in controlled (classroom setup) as well as an uncontrolled environment (emails). Based on their personal information, the 19 respondents were divided into two groups (Table 5-1, 5-2):

- Basic (the group consisted of people with basic knowledge of RS and some experience in slum related studies)

- Advanced (people with high experience in RS and practical experience, in terms of visits to a variety of slum contexts. This group also consists of people with proven record (publications) of RS based slum mapping).

Table 5-2 shows the region respondents belonged to, number of region wise responses and the slum context they were most familiar with.

Table 5-1 Number of respondents grouped based on their experience in remote sensing and slum mapping.

\begin{tabular}{lc}
$\begin{array}{l}\text { Remote sensing } \\
\text { experience }\end{array}$ & $\begin{array}{c}\text { Number of } \\
\text { respondents }\end{array}$ \\
\hline Basic & 10 \\
Advanced & 9 \\
\hline
\end{tabular}


Table 5-2 List of region of origin and countries referred to by respondents in the expert survey.

\begin{tabular}{lcl} 
Region & $\begin{array}{c}\text { Number of } \\
\text { respondents }\end{array}$ & Most familiar slum context \\
\hline Asia & 3 & India, Phillipines \\
Sub-Saharan Africa & 8 & Kenya, Rwanda, Tanzania, Ghana \\
Latin America & 2 & Jamaica, Argentina \\
Europe & 6 & $\begin{array}{l}\text { Tanzania, India, South Africa, Indonesia, } \\
\text { Bangladesh, Venezuela }\end{array}$ \\
\hline
\end{tabular}

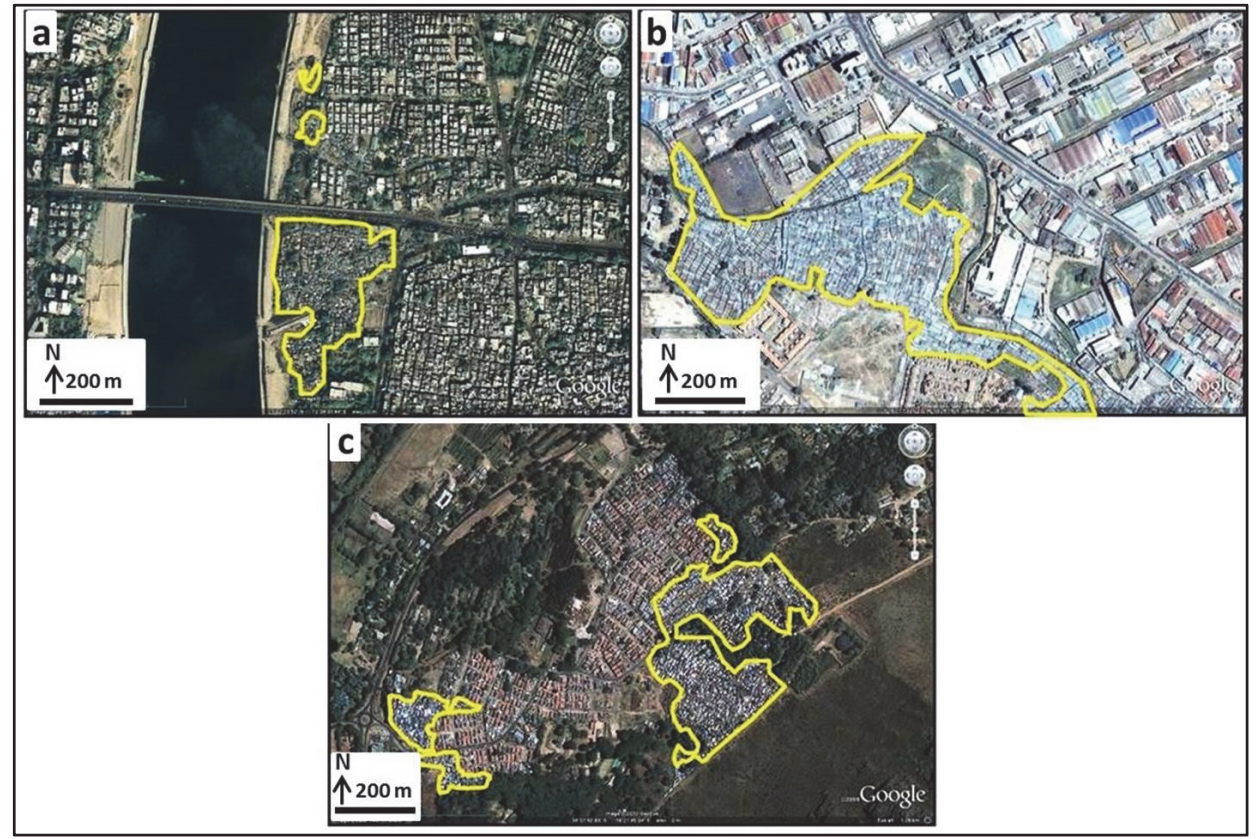

Figure 5-1 Slum delineation in different contexts by a single expert in the cities of a) Ahmedabad (India), b) Nairobi (Kenya), c) Cape Town (South Africa). Source: Google Earth.

\subsubsection{Statistical analysis}

To study both the agreement between observed slums as well as the uncertainty existing in visual interpretation of slums, we use relatively simple measures. First, the agreement in terms of the extent of slums delineated by experts in the three contexts was calculated. For convenience in analysis and better understanding, the agreement range of experts was divided into five intervals. The upper most interval with agreement of 15 or more experts $(>80 \%$ ) was taken as the area with high likelihood of being a slum and the corresponding percentage of total identified slum area was determined (Table 5-3). To account for over-estimation of slum area by some experts, the area identified by three or less experts was subtracted from the total. The resulting 
percentages gives an estimation of most likely and unlikely slum areas. In case of Nairobi, proportion of area was also calculated after removing the overestimated area resulting from different windows extents used while digitizing (Figure 5-5). This was done by cropping the agreement layer with a common boundary that included all the digitizations towards the south.

Secondly, uncertainty for delineated objects was modelled to estimate existential and extensional uncertainty. A common way to model uncertainty in object oriented studies is the use of random sets (Zhou and Stein 2013, Zhao et al. 2010). Random sets are based on probabilistic functions, and specify the probability that an object is present at a location. Their application leads to the support of a slum, i.e. the area on the ground where a slum could potentially be, the core of a slum, i.e. the area where the slum is with certainty, and intermediate areas where a slum is present with different degrees of likeliness.

We first consider a hypothetical situation shown in Figure 5-2, where the core set $(C)$ is most certainly a slum, and the region $U$ represents an area where possibly the slum is as well. This situation, could result in three scenarios for delineation: 1) slum delineation encloses the actual slum boundary completely, i.e. it overestimates the total area; 2 ) slum delineation is inside the actual slum boundary, i.e. it misses parts of the fringes and 3) slum delineation is exactly the same as the actual slum boundary. A boundary should specify where a slum becomes a non-slum. Uncertainties may enter, for example, if a road next to a slum is included in the slum or not. Thus the region $C$, represents the area where there is maximum agreement or certainty of it being a slum and receives the value one, whereas $U$ could be represented by different values between one and zero, and the white area is non-slum and receives the value zero. 


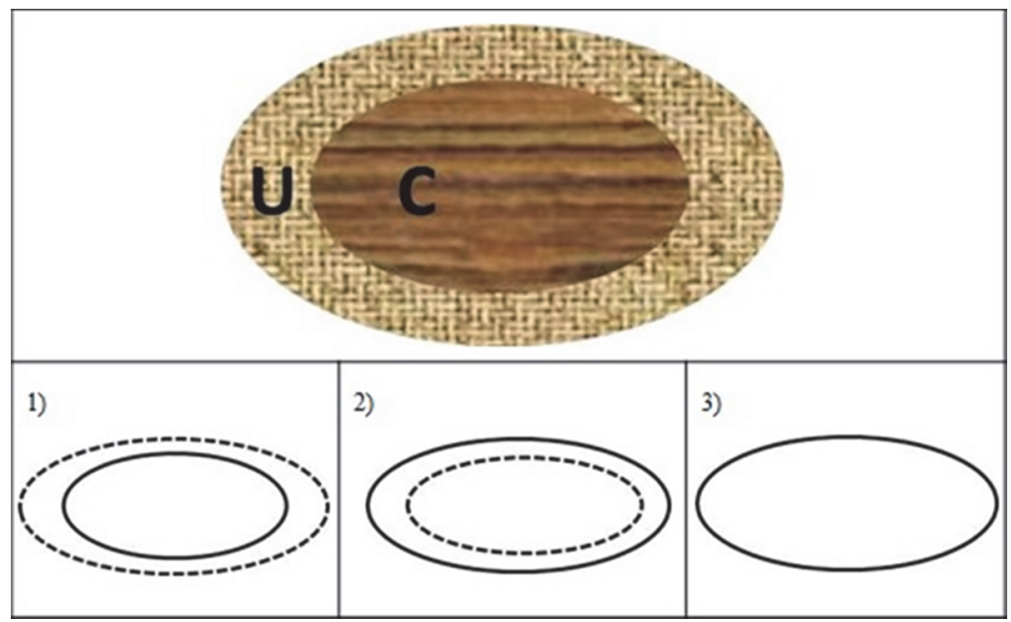

Figure 5-2 Different forms of extensional uncertainty: 1 ) slum delineation encloses the actual slum boundary; 2 ) slum delineation inside the actual slum boundary; 3 ) slum delineation same as the actual slum boundary.

To model the uncertainty in terms of random sets for the current study, we considered $n=19$ polygons $O_{i}, i=1, \ldots, n$, defined in the image $A$ by the group of $n$ expert users selected at random from a larger group. Out of these polygons, indicator functions $I_{i}(s)$ were defined with values at each location $s \in A$, where $I_{i}(s)=1$ if $s \in \mathrm{O}_{\mathrm{i}}$, and 0 if $s \notin \mathrm{O}_{\text {i. }}$. In this way $n$ indicator functions were obtained. The random set that covers the slum area was then obtained as the average of the functions $I_{i}(s)$, i.e. $I(s)=1 / n \Sigma_{i=1 . . n} I_{i}(s)$. Values of $I(s)$ ranged between 0 and 1 with steps equal to $1 / n$. The value 0 occurred if none of the experts identified an area as slum, whereas the value 1 occurred if each of the experts identified it as a slum. Thus, uncertainty exists for values between 0 and 1 . Such uncertainty could be attributed to extensional uncertainty as some experts may have, for example, assigned a part of a bordering road to a slum area (assigning it the value 1), whereas others interpreted it as the non-slum area (hence assigning it the value 0). Some areas also resulted in a low $I(s)$ value because there was existential uncertainty: possibly only a few experts identified it as a slum. In this study, we explored different values of $I(s)$ for an area to be existentially or extensional uncertain.

The selection of the $n$ experts was done as described above, and hence in order to apply the essentials of random sets, we applied a bootstrap procedure. Under this procedure, sampling is done with replacement many $(B)$ times to estimate the variability of the mean and variance of model outputs (Champagne et al. 2014). Out of the $n$ experts, a random selection was made with replacement of $n$ experts and the coverage function $I^{(1)}(s)$ was made by aggregating the $n$ individual coverage functions as before. This procedure was repeated $B$ times, yielding coverage functions $I^{(b)}(s)$, for $b=1, \ldots, B$. The value 
of covering function ranged from 0 to 1 for the random region $\mathrm{R}$. The average of the $I^{(b)}(s)$ was the bootstrap estimate of the random set. Additionally, the standard deviation (sd) of $I^{(b)}(s)$ gave a measure of boundary deviations and showed the uncertainty on the borders and confidence in the axis. In the current study, the value of $B$ was set to 100 .

\subsubsection{Group-wise and city-wise comparison of the delineations}

A simple linear model was used to study the area deviations in delineations based on different contexts and experience. Under the null hypothesis, it was assumed that there was a significant difference between the advanced and basic respondents. An additional analysis of variance was conducted to study the significance of experience and the cities only for the advanced group. The $p$-value of the $t$-test for each variable, the $F$ value and its significance level and the squared correlation $\left(R^{2}\right)$ were calculated.

\subsection{Results}

Figure 5-3 show the agreement maps derived from the slum delineations of 19 respondents. The agreement levels range from a minimum value of 1 (delineated by 1 respondent) to a maximum of 19 (delineated by all 19 respondents).

\subsubsection{Area of maximum agreement level}

For Ahmedabad, $15 \%$ of the total identified slum area was delineated by 15 or more respondents. After removing the area delineations by 3 or less respondents, the proportion increases to $42 \%$. Such delineations generally show the over-estimation of slum areas as shown in Figure 5-3. For Nairobi, the proportions of area before and after removal of over-estimated area were equal to $48 \%$ and $61 \%$, respectively. After removing the over-estimated area resulting from different windows extents, the proportion increased to $73 \%$. Figure 5-3c shows the agreement map for Cape Town. $47 \%$ of area was identified by more than 15 experts. The percentage increased to $61 \%$ after removal of probable over-estimated area. 
Table 5-3 Percentage of identified slum area at maximum agreement level. The percentage of adjusted area is the area after removing the over-estimated area identified by three or less respondents.

\begin{tabular}{lll}
\hline \multirow{2}{*}{ Cities } & \multicolumn{2}{c}{ Levels of agreement } \\
\cline { 2 - 3 } & $\begin{array}{l}\text { 15-19 experts } \\
\text { (\% area) }\end{array}$ & Adjusted area (\%) \\
\hline Ahmedabad & 15 & 42 \\
Nairobi & 48 & 61 \\
Cape Town & 47 & $61 / 73$ \\
\hline
\end{tabular}

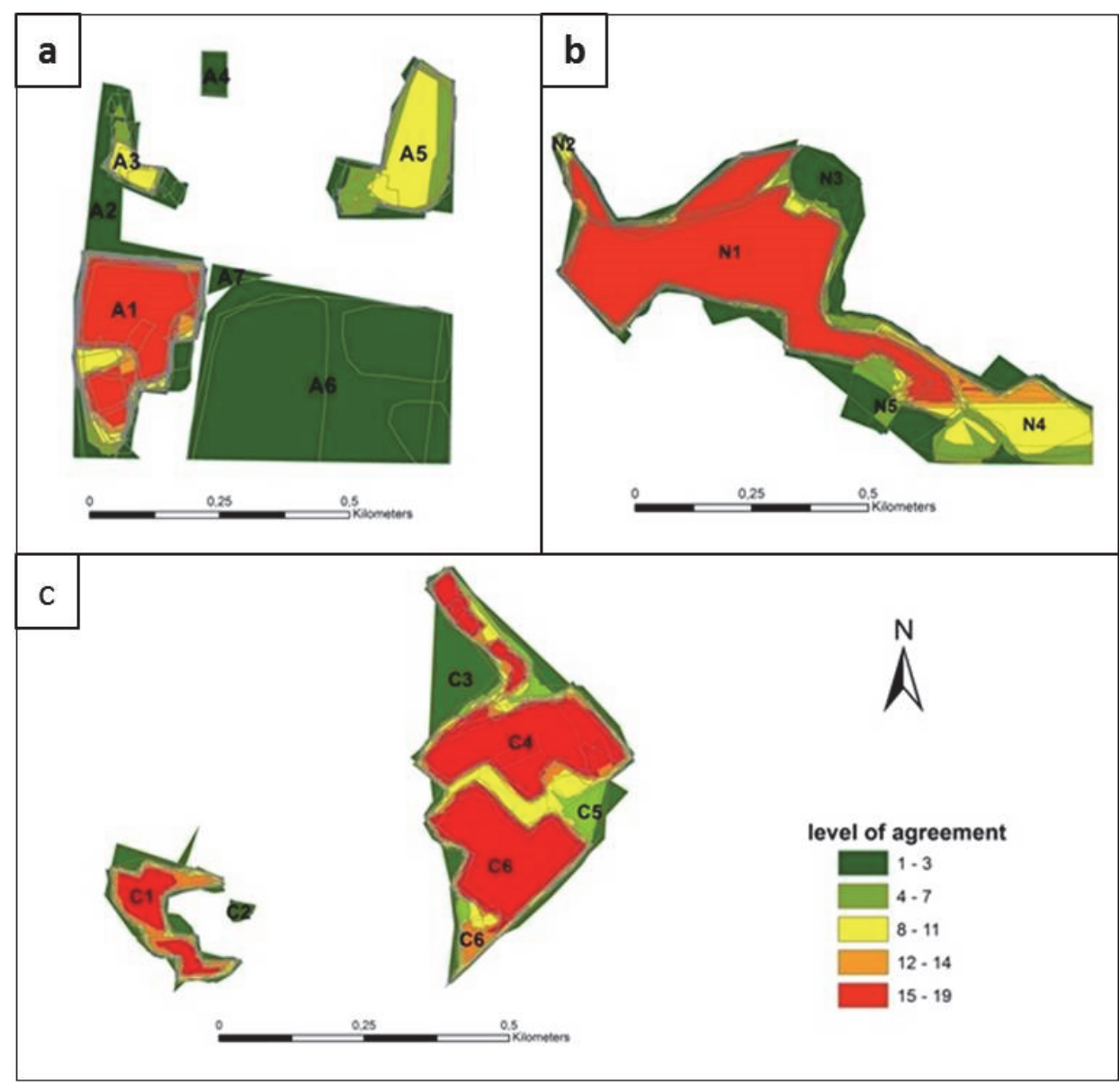

Figure 5-3 Agreement level layers of slum delineations by 19 experts for the three contexts (a) Ahmedabad (India), (b) Nairobi (Kenya) and (c) Cape Town (South Africa). 


\subsubsection{Modelling uncertainty using random sets}

\section{a. Nairobi}

Figure 5-4 shows the bootstrap estimates of the random set (Figure 5-4a and 5-4b) for Nairobi. Figure 5-5 shows the overlays of 19 delineations on the Google earth snapshot with the magnified view of selected areas. Analysis based on random set results is discussed as follows:

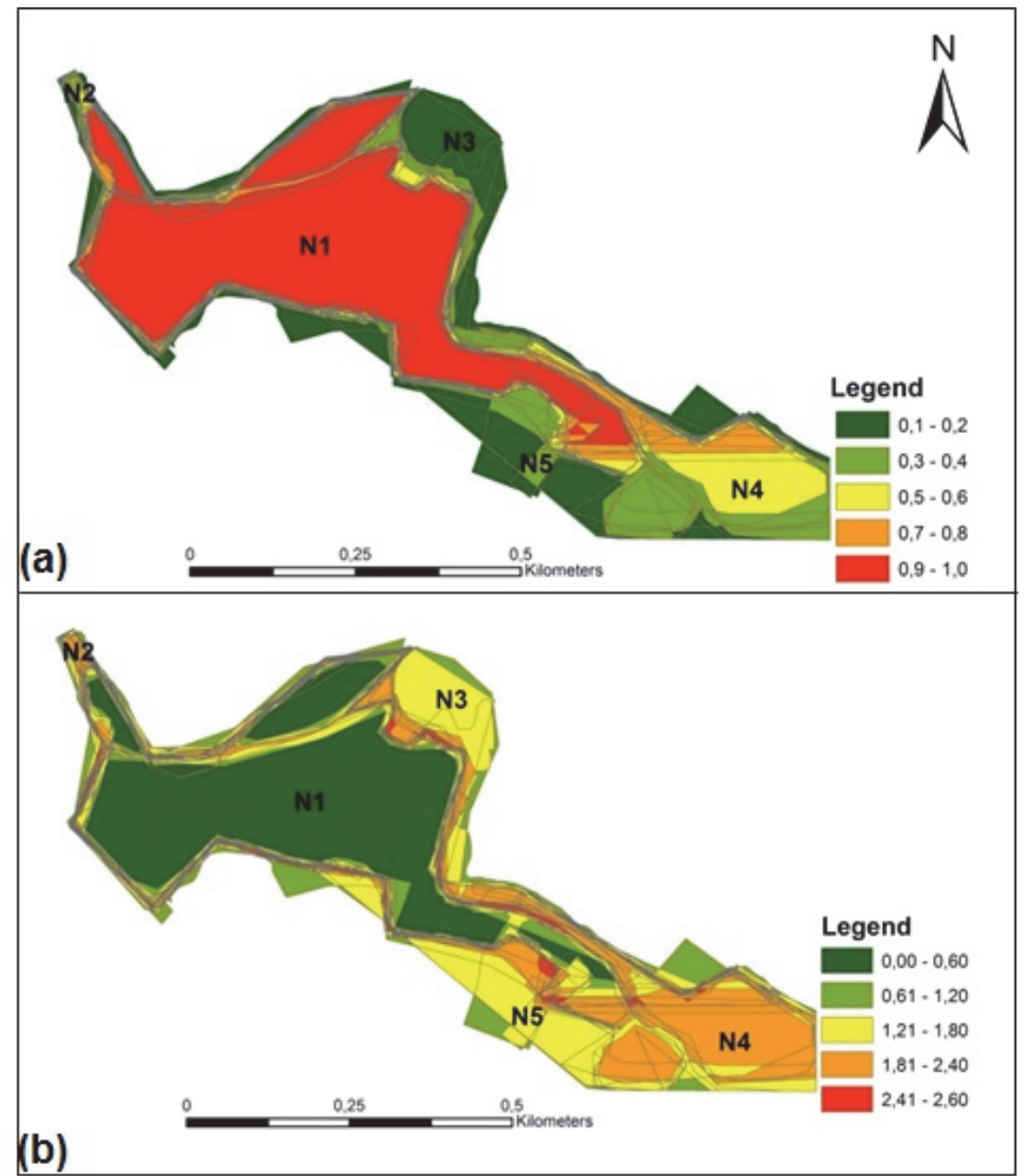

Figure 5-4 The bootstrap estimates of random set for Nairobi: (a) the random set model shown by its covering function; (b) the standard deviation of random set.

- The red area at the centre, N1, in Figure 5-4a indicates a slum area with a high certainty $(I(s)>0.9)$. Figure $5-4 \mathrm{~b}$ also shows this area as green, signifying low sd values, hence consistently identified as a slum area by the majority of 
respondents. The fringes of $\mathrm{N} 1$ that can be considered a transition zone, however, show lower $I(s)$ values, due to differences in boundary placement by respondents and hence is considered extensionally uncertain. A small yellow polygon between $\mathrm{N} 1$ and $\mathrm{N} 3$ in Figure $5-4 a(0.5<I(s)<0.6)$, is a classic example of extensional uncertainty where respondents struggle with including a small open area embedded within the slum buildings in the delineation (Figure 5-5b). The majority of respondents clearly use linear features, such as a road bordering the slum in the south and drainage in the north.

- Area N2 indicates extensional uncertainty resulting from low contrast with the adjoining buildings of non-slum area (Figure 5-4a). A careful examination also shows a fair amount of generalization by different respondents resulting from high to very low precision (Figure 5-5a). Thus, deviations could be attributed to conceptual differences and generalization introduced by different experts.

- Area N3 indicates existential uncertainty. Some respondents included the green polygon (Figure 5-4a) in the slum, whereas it was excluded by most respondents. There is confusion as concerning whether to include the open area in the slum (Figure 5-5b). The relatively high sd values (Figure 5-4b) show disagreement among respondents for this area.

- Area N4 shows that different image extents were used by the interpreters (Figure 5-4, 5-5). Clearly, the respondents did not stick to the provided extent of snapshot resulting in under or over estimation of areas towards the bottomright of the image. Thus the southernmost border of N4 is not consistent and has a large effect on the results.

- For area N5, the southern border also shows deviations, again in terms of boundary placement. Although, the relatively high sd values $(>1.21)$ are due to inclusion or exclusion of a vegetated area and a parking lot for this area.

A relatively crisp boundary with a distinctly different feature, e.g. a road, a river, an industrial area or a planned residential, tends result in a higher level of agreement expressed as a narrow transition zone, whereas a boundary between neighboring urbanized areas with similar characteristics tends to result in higher sd values (Figure 5-4b). In such cases the effect of a combination of existential uncertainty and variable levels of generalization can be seen (Figure 5-5). 


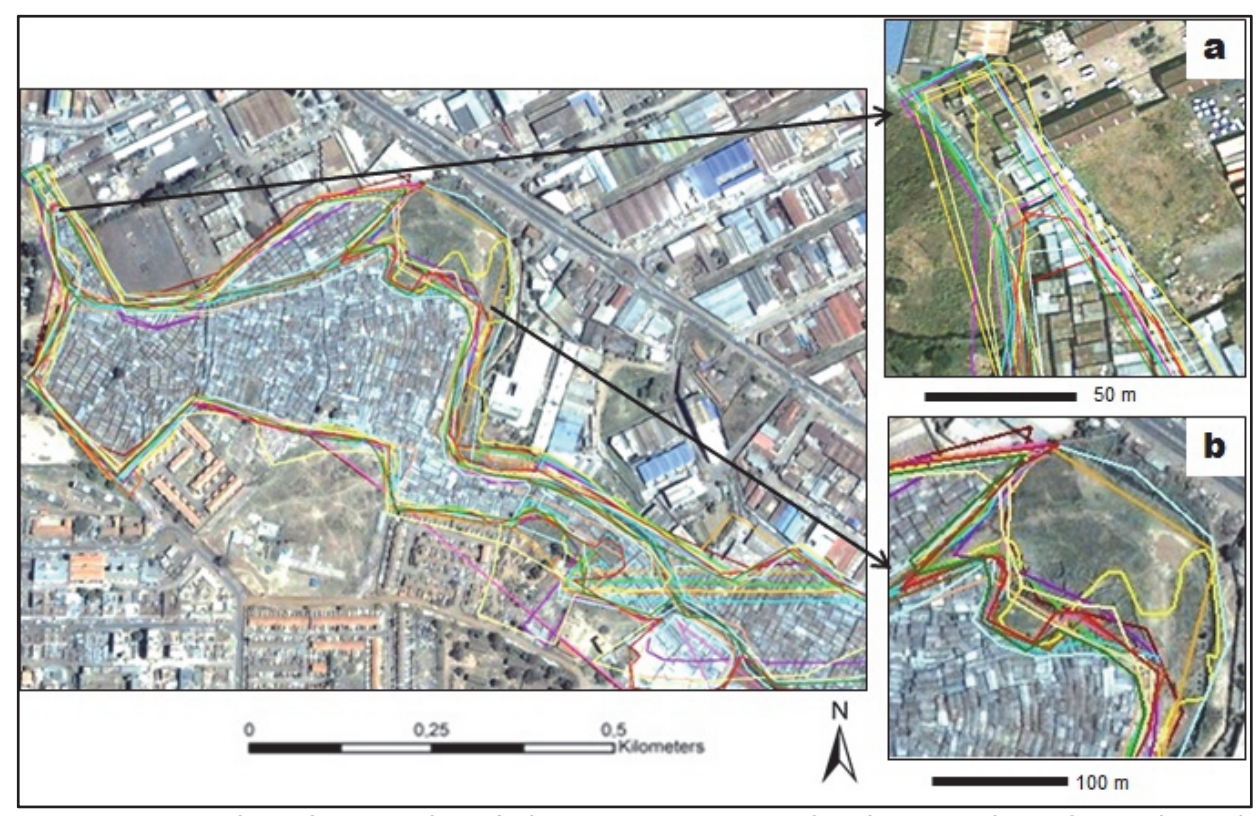

Figure 5-5 Overlay of respondents' slum interpretations for the snapshot of Nairobi and the magnified view of two areas: (a) boundary deviation due to generalization; (b) deviation resulting from inclusion/exclusion of fringe in Nairobi.

\section{b. Ahmedabad}

Figure 5-6 shows the bootstrap estimates of the random set (5-6a and 5-6b) for Ahmedabad. The overlays of 19 delineations on the Google Earth snapshot are shown in Figure 5-7. The analysis based on random sets shows the following results.

- The area A1 in the west, in different colors (red, yellow, orange and green) indicates a slum. The red area is a slum with certainty, whereas the size is extensionally uncertain as is represented by different colored polygons (Figure 5-6a). The red color corresponds with $I(s)$ values between 0.9 and 1 , and hence identifies the core of the slum. Extensional uncertainty is due to various reasons: confusion on whether to include a big building, trees or vacant land to the fringes of the slum or to exclude those from it. The sd map also shows a relatively high deviation on the fringes (Figure 5-6b), whereas there is maximum consensus on the core area of A1 (dark green polygon).

- The green colored polygon $A 2$, also linking $A 1$ and $A 3$ in Figure 5-6a indicates existential uncertainty. It appears like a patch of land which some experts assumed to be part of $A 1$, although there is little consensus on this area being a slum also proved by low sd values in Figure 5-6b. 
- A green rectangle at the top, A4 in Figure 5-6a indicates existential uncertainty.

- A big green area bottom right, A6 and A7 in Figure 5-6a shows existential uncertainty.

- A yellow-green area top right A5 in Figure 5-6a indicates the core area displayed in yellow is extensionally uncertain. The relatively high sd values ( $>$ 1.81) indicate a low agreement on this area being a slum, and hence to existential uncertainty.

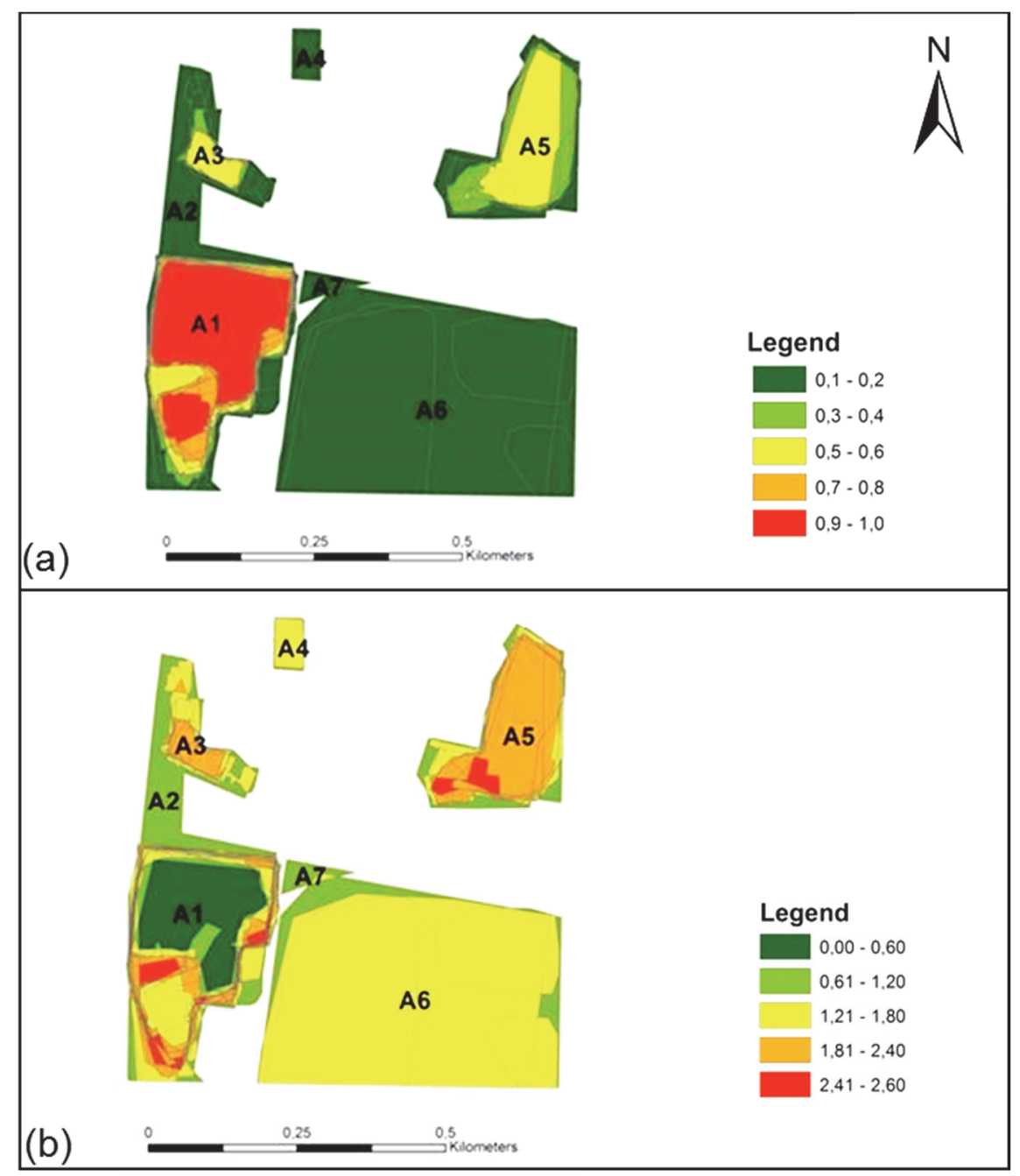

Figure 5-6 The bootstrap estimates of the random set for Ahmedabad: (a) the random set model shown by its covering function; (b) the standard deviation of random set. 
Most experts agree on one settlement along the river to be a slum (A1), although there is extensional uncertainty, as deviations exist in boundary placements (Figure 5-6b). There are other areas delineated as slums where relatively less agreement exists, whereas there are areas with least agreement. Most experts identify one big polygon as a slum (core of A1). The contrast with the adjoining areas is a leading factor for maximum agreement and low uncertainty in this case. Although there are deviations in terms of placing the boundary, transition zones are either wide or narrow. This once more points to placing boundaries in the case of including or excluding an open area, vegetation or buildings next to a slum inside the slum polygon. Delineations may depend on the expertise and context-knowledge of a respondent also. For example, some respondents have identified the old city centre (Figure 5-7) in Ahmedabad (A6) as slum, being clearly unacquainted with the Indian context.

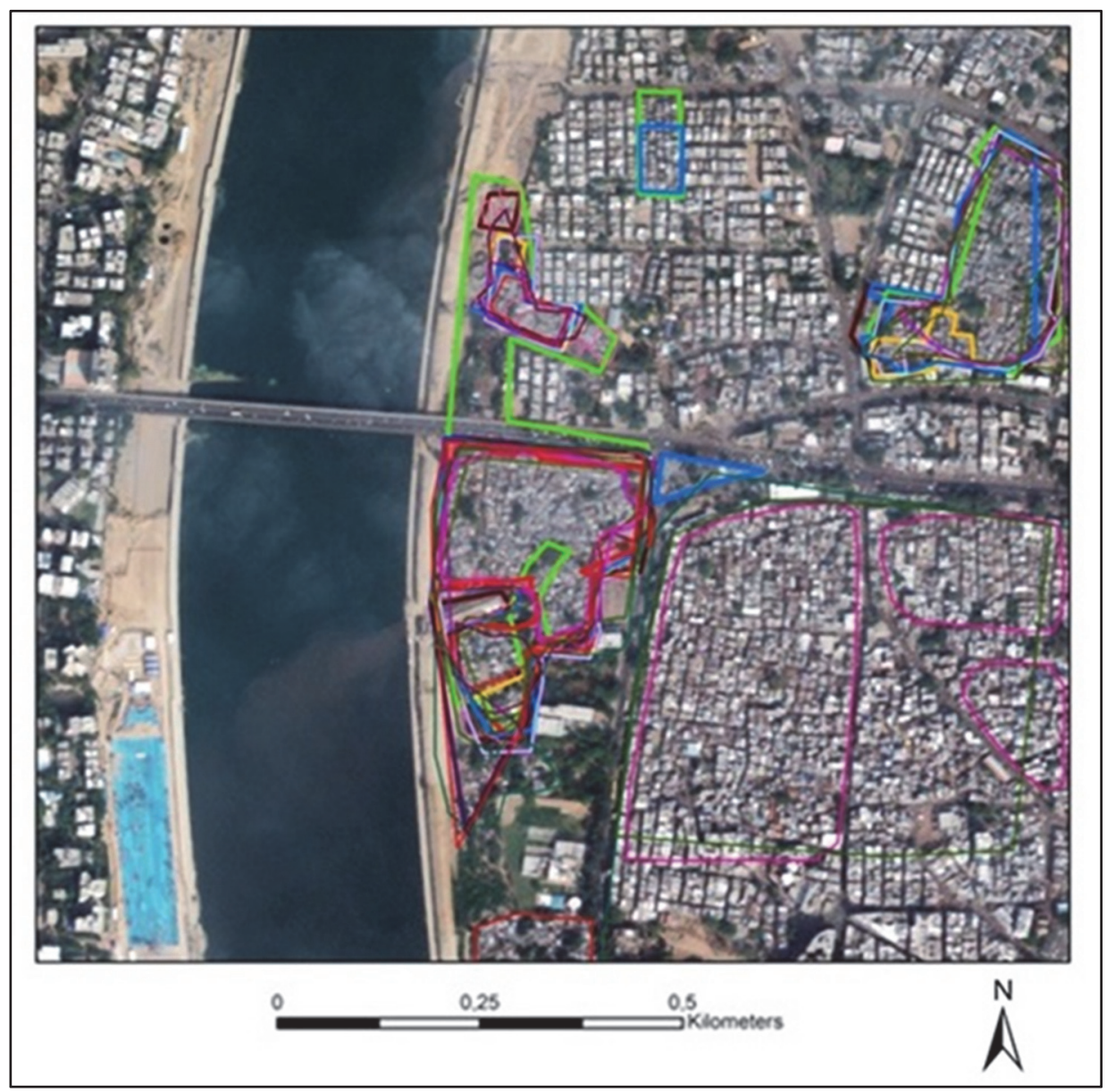

Figure 5-7 Overlay of respondents' slum interpretations for the snapshot of Ahmedabad. 


\section{c. Cape Town}

Figure 5-8 shows the bootstrap estimates of the random set ( $5-8 a$ and $5-8 b$ ) for Cape Town. Figure 5-9 shows the overlays of 19 delineations on the Google Earth snapshot. Analysis of the random set results lead to the following:

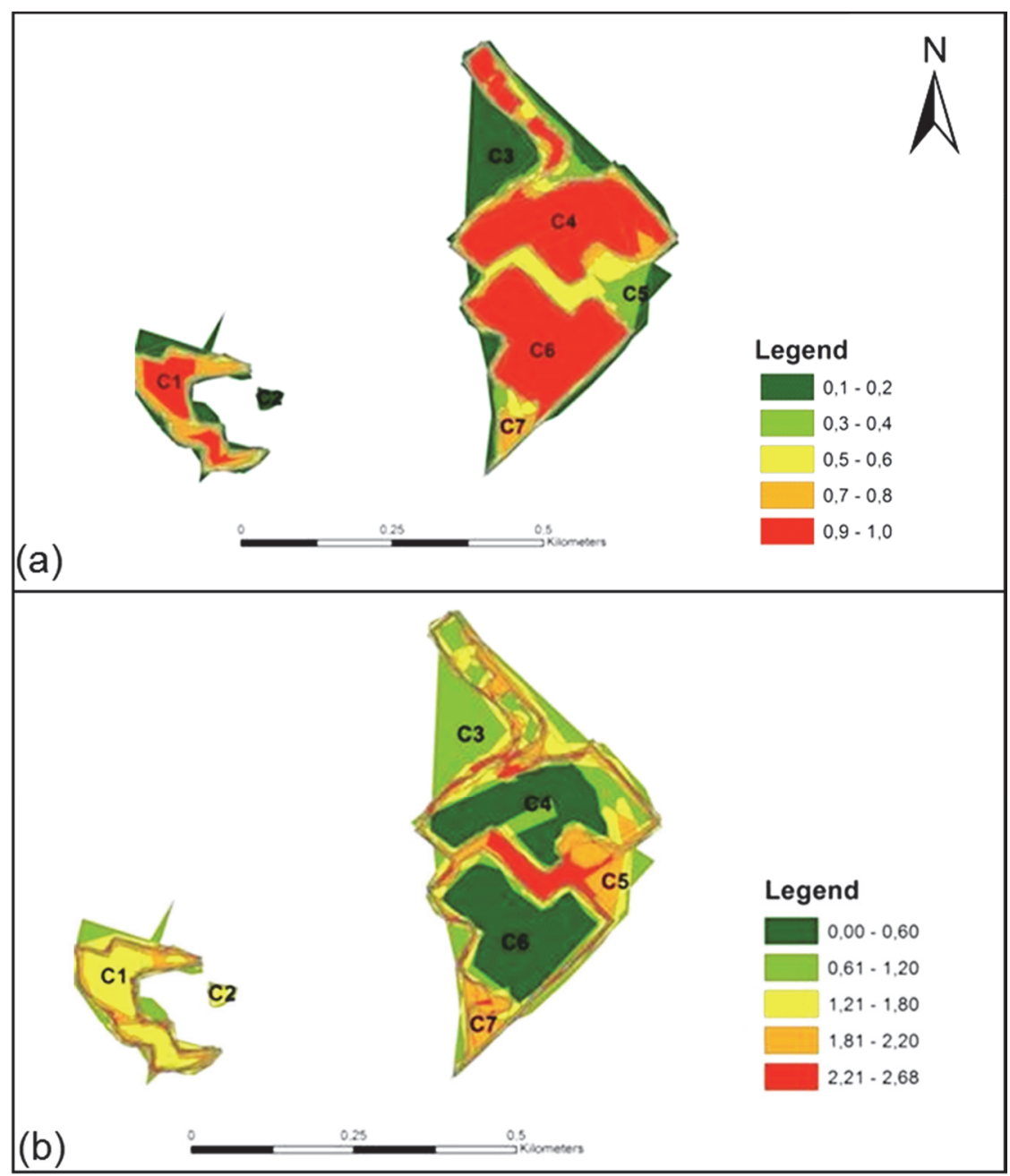

Figure 5-8 The bootstrap estimates of random set for Cape Town: (a) the random set model shown by its covering function; (b) the standard deviation of random set.

-The red areas at the core, C1, C4 and C6 in Figure 5-8a are identified as slum areas with a high certainty. There is little existential uncertainty at the green polygon close to the larger multi-colored polygon on the left, C2. Extensional uncertainty is predominant. We notice the difference between the core $(I(s)>$ $0.9)$ and the uncertain areas around it $(I(s)<0.9)$ (Figure $5-8 a$ ). 
- Delineation of area C3, identified by one expert, is most likely is the result of lack of digitizing skills of the respondent or purely negligence. The polygons to the right of $\mathrm{C} 3$, colored in red, orange, yellow and green, show slum areas identified as patches by some respondents who carefully included only buildings and excluded vegetation. A similar behavior is observed at areas C5 and $\mathrm{C7}$ where respondents mainly do not agree on including or excluding the vegetation (Figure 5-8a). The yellow area between areas C4 and C6 (Figure 5$8 a$ ) is also caused by the presence of vegetation where respondents have variable views, clearly demonstrated by the high sd values (Figure 5-8b). The extensional uncertainty in this case could be attributed once more to confusion caused by vegetation patches (Figure 5-9). Additionally, boundaries are generalized, hence resulting in extensional uncertainty on the fringes. The dark green polygons at the centre ( $\mathrm{C} 6$ and $\mathrm{C} 4$ ) are identified as slums with the most stable boundary as shown in Figure 5-8b.

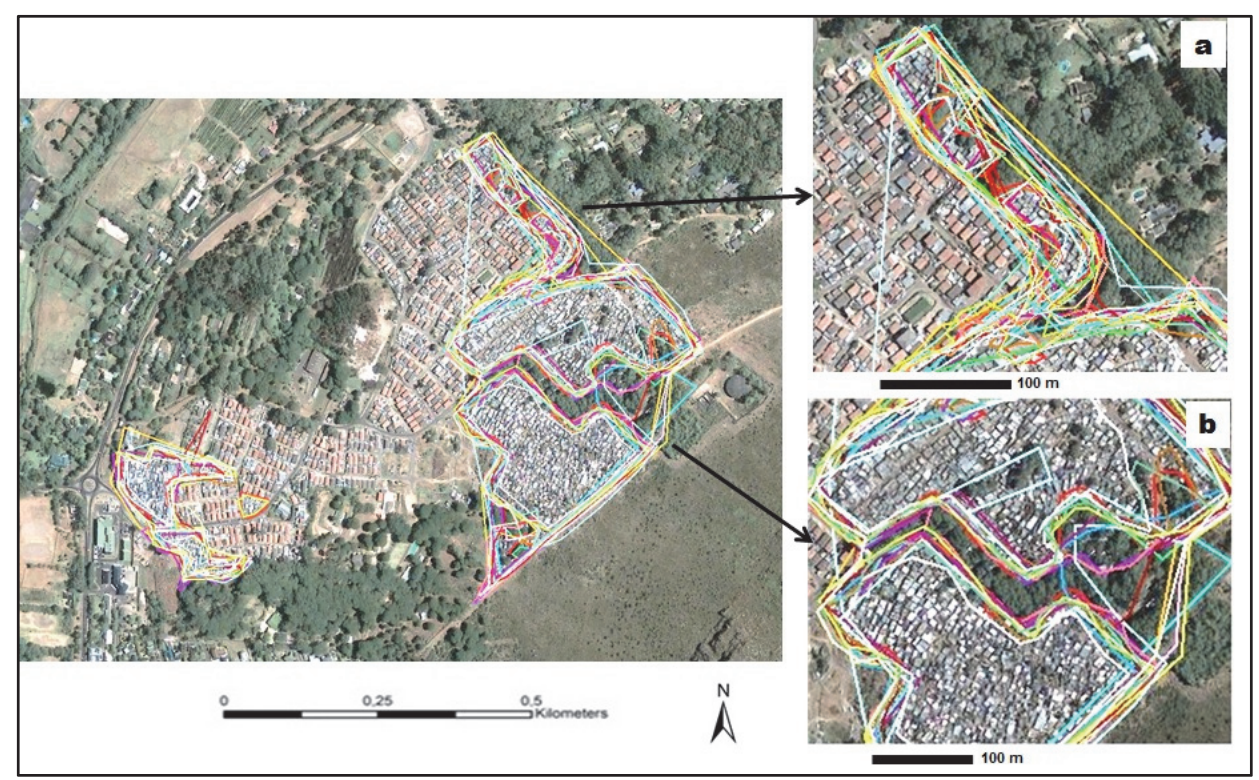

Figure 5-9 Overlay of respondents' slum interpretations for the snapshot of Cape Town and the zoomed-in view of two areas ( $a$ and b) showing boundary deviations resulting from inclusion or exclusion of vegetation.

\subsubsection{Difference in delineations of the two groups and cities}

It was hypothesized that advanced interpreters will tend to express less variation in the delineations than basic interpreters. Figure 5-10 shows descriptive statistics for the interpretations for each group in each context. The average area, minimum area and sd are similar for both the groups except the maximum area in Nairobi. A similar trend exists across Ahmedabad and Cape Town, as the maximum area is always higher for the basic group. 


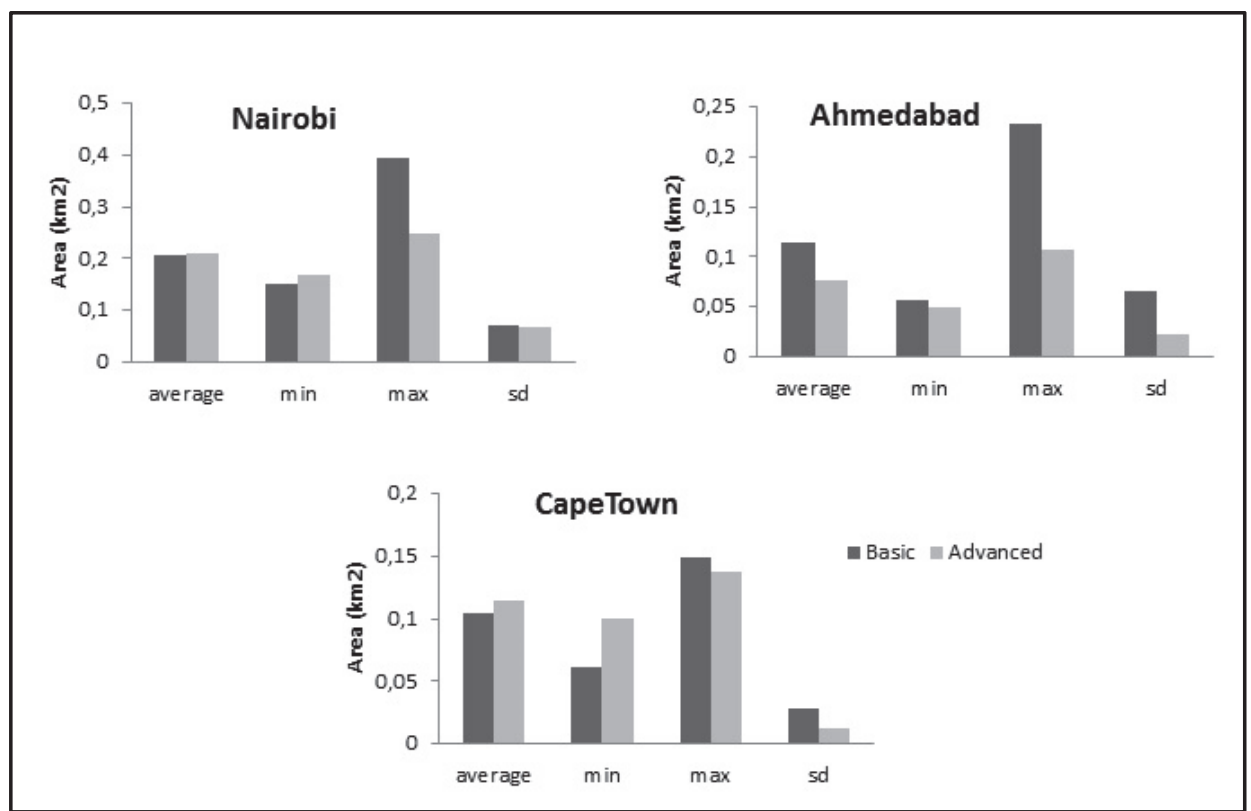

Figure 5-10 Average, minimum, maximum and standard deviation of areas delineated by the advanced (light) and basic (dark) group for Nairobi, Ahmedabad and Cape Town.

The statistical analysis (Table 5-4) shows that the advanced group has somewhat (non-significant) lower area estimates as compared to the basic group ( $p=0.519$ ). The value of $R^{2}$ equals 0.42 , i.e. $42 \%$ of the variability in area can be explained by the model. There is a significant difference between Ahmedabad and Nairobi $(p<0.001)$, but not between Nairobi and Cape Town. Hence, the difference between Ahmedabad and Cape Town is also significant. This was confirmed by analysis of variance (Table 5-5). Here also, experience is not significant $(p=0.504)$, but significance is present among the cities within the advanced group.

Table 5-4 Linear relations between area and the explanatory variables, with respect to different cities and experience.

\begin{tabular}{lllrl} 
Parameters & Est. $^{\mathbf{a}}$ & SE $^{\mathbf{b}}$ & t value & $\boldsymbol{p}$-value \\
\hline Intercept & 0.098896 & 0.009142 & 10.818 & $5.0 \mathrm{e}-15^{* * *}$ \\
Ahmedabad-Nairobi & 0.065989 & 0.011339 & 5.820 & $3.5 \mathrm{e}-07 * * *$ \\
Nairobi-Cape Town & 0.012721 & 0.011339 & 1.122 & 0.267 \\
Experience & -0.006015 & 0.009271 & -0.649 & 0.519 \\
\hline
\end{tabular}

${ }^{\mathrm{a} E s t i m a t e d}$ coefficient

bStandard error

*** indicates $\mathrm{p}$-value under a 0.001 significance value 
Table 5-5 ANOVA of area in relation to experience and cities within the advanced group.

\begin{tabular}{llllll} 
& Df & SSq $^{\mathbf{a}}$ & MSq $^{\mathbf{b}}$ & F value & $\boldsymbol{p}$-value \\
\hline experience & 1 & 0.00051 & 0.000514 & 0.454 & 0.504 \\
experience:city & 4 & 0.05355 & 0.013387 & 11.820 & $7.21 \mathrm{e}-07 * * *$ \\
\hline
\end{tabular}

asum of squares

${ }^{\mathrm{b}}$ Mean square

$* * *$ indicates $\mathrm{p}$-value under a 0.001 significance value

The statistical analysis provides an understanding of the differences that can be attributed to contexts. A particular context may be easier for delineating slums compared to other. For example, in case of Nairobi, the slum has a clear contrast to non-slum areas. Other than confusion in placing the boundaries on the fringes, there seems to be agreement amongst most experts on a large part of slum area (Figure 5-4). Cape Town is also reasonably easy as much of the confusion is mainly related to inclusion/exclusion of vegetation. Ahmedabad, India seems to be more complex than Nairobi and Cape Town. With the specific example of the old city centre (Figure 5-7), which some respondents mis-identified as a slum due to morphological similarity to densely packed slum areas. Thus, based on our analysis, experience is not significant i.e. the two groups give the same assessments. Though there is strong, significant difference between the three cities.

\subsection{Discussion}

\subsubsection{Factors used to distinguish slums from non-slum areas}

An apparent issue is the quality of the data in this study. Here the main issue to consider is the way that delineation is being done and what major problems are. In order to investigate this, respondents were asked on which basis they primarily delineated slums from imagery. The main reoccurring factors in the three contexts were obviously building size, roof material, absence of roads, irregular roads, lack of vegetation and open spaces, density (compactness), irregular shape of settlement, association with neighbouring areas, texture and locality. The combination of these indicators, however, varied from one context to another.

\subsubsection{Factors leading to difficulties in slum interpretation}

Based on a limited response, context-wise factors are summarized in the following sub-sections.

\section{a. Nairobi}

Four respondents answered this query for Nairobi. The factors causing difficulties were: 
- $\quad$ confusion between residential and commercial area along the borders.

- uncertainty about the accuracy of the border due to time limitation (performed in class-room setting).

- $\quad$ confusion regarding the extent of the Google snapshot.

In addition to other factors, the source of maximum variation can be attributed to different window extents used while digitizing rather than actual identification. Somewhat remarkably, one of the respondents, cited Nairobi as the easiest of the three contexts.

b. Ahmedabad

Nine respondents answered this query, indicating that respondents found the Ahmedabad context comparatively most challenging. The responses given to this query are summarized as follows:

- A majority of respondents found tree cover as an inhibition for accurate delineation. Vegetation, in some cases, covers the border completely and hence leads to confusion of placing borders.

- Conceptual difficulty exists in terms of including less dense areas in the slum delineation or not. There was confusion on some areas being residential or market areas.

- Lack of context knowledge was identified as an important limiting factor by some respondents.

- The order of buildings looks similar for slum and non-slum areas.

A common cause is therefore the high complexity, where non-slum areas morphologically resemble slum-areas.

\section{c. Cape Town}

Seven respondents answered the query in case of Cape Town. The responses are summarized as follows:

- Tree cover was identified as a major problem for delineation by almost all respondents.

- Houses below trees were sometimes completely hidden. 
- There was an apparent lack of experience and context-knowledge among the respondents.

- $\quad$ Fuzzy boundaries exist between slum and formal housing areas.

The main obstacle for an accurate slum delineation in Cape Town was identified as vegetation.

Analysis of the uncertainty was done in this study mainly on the basis of random sets. This was considered a relevant way of doing so, as we could consider the group of nineteen respondents as a random sample out of the 50 experts. Moreover, bootstrapping results were a key way to indicate the variability and uncertainty in the boundary areas. As an alternative, we could have used fuzzy sets, based on fuzzy logic. Fuzzy logic is based on membership functions that relate identified objects with classes, leading to a degree of belongingness. We see this as a less suited procedure for the current study, as the choice of membership function is highly arbitrary and setting parameter values is far from being straightforward (Zhou and Stein 2013). In our view, random sets are the best choice at present to model the extensional and existential uncertainty of slums as delineated by experts from remote sensing images.

Clearly, a study of slum interpretations in three cities is rather limited to cover the variation of situations that can be found across all cities within the less developed world. The results obtained in this study suggest that accurate interpretations of slums may be best produced by those most familiar with specific contexts. These could be local geo-information professionals but could also include citizen organizations and Non-Government Organizations (NGOs) working with slum dwellers or an alliance of professional and non-professional interpreters. As OOA approaches also require considerable tuning to local contexts, access to local knowledge is likely to be an important aspect of the accuracy of OOA based slum maps.

\subsection{Conclusions}

In this study, we studied deviations in slum identification and their delineations as observed from VHR images of Ahmedabad (India), Nairobi (Kenya) and Cape Town (South Africa). Existential and extensional uncertainties of slums were modelled in terms of random sets and a bootstrap sampling method is applied to compute the confidence in the various delineations. Additionally, we identified the factors of the built environment that experts use to distinguish slums from non-slum areas and factors leading to deviations in slum identification in the three contexts and hence the reasons for uncertainties. 
From the study we conclude that in all contexts there is a considerable variation in the delineated areas suggesting that a slum has uncertainty, both on the basis of the context, e.g. existential uncertainty, and of sharply delineating it, i.e. extensional uncertainty. We also conclude that the level of experience is not a significant determinant of slum identification and delineation accuracy. A highly significant difference exists between delineations of the Indian and SubSaharan African cities. Even after removing the most unlikely areas we found that the most likely slum areas range between 42\% (Ahmedabad), 61\% (Nairobi and Cape Town) and 73\% (Nairobi, after adjusting the extent) of the total delineated areas. The distinction between slum and non-slum areas is more evident and more clearly identifiable on the images of Nairobi and Cape Town. The difference with Ahmedabad is that the morphology of the city is denser, buildings are smaller, and that on a remote sensing image the large historic city quarter is similar to a slum area. Local knowledge therefore may play a greater role in extracting slum data from images of Ahmedabad than in the two other cities. 
Chapter 6

\section{Synthesis}




\subsection{Conclusions}

The main objective of this study was to develop and test remote sensing based methods for identifying and characterizing slum areas using very high resolution (VHR) satellite imageries. In this section, the conclusions of research finding are presented for each objective referred in section 1 .

\subsubsection{Research objective 1}

Development of an ontological framework to conceptualize slums using morphological indicators of the built environment.

Remote sensing based methods may be used for a rapid inventory of the location and physical composition of slums. Such methods, particularly objectoriented image analysis approaches (OOA), require a robust conceptualisation of slums that is on the one hand sufficient to serve as basis for slum identification and classification, but on the other hand is flexible enough to deal with the large variability in slum characteristics. Taking UN-HABITAT's definition of slum household as a basis, this study conceptualized slums using an ontological approach appropriate for use with VHR images. We focused particularly on physical aspects that may be derivable through remote sensing, i.e. identifying which morphological properties of the built environment could be used to distinguish slums from non-slum areas.

In chapter 2, we developed an ontological framework to conceptualize slums in VHR satellite images using input collected through a semi-structured survey from fifty domain-experts from sixteen countries. The results of the survey showed that experts use eleven basic factors to identify slums from VHR imagery: building size, roof material, absence of roads, irregular roads, lack of vegetation and open spaces, density (compactness), irregular shape of settlement, association with neighboring areas, texture and locality. These factors form the basis for the Generic Slum Ontology (GSO) comprising concepts at three spatial levels: object, settlement and environs. The object level refers to the characteristics of the main physical components of the settlement, i.e. the variables that relate to buildings and roads within a slum (e.g. building size and shape; regularity and width of roads). The settlement level addresses the overall form, shape and density of a slum. The environs level refers to the location of a slum with respect to surrounding areas in terms of neighborhood characteristics and hazard-prone zones.

The GSO provides a framework for understanding the relation between image characteristics and actual slum characteristics, as it provides the opportunity to integrate local knowledge through the selection of relevant indicators and by adjusting the parameters for each selected indicator to local conditions. As an example of local adaptation of GSO and OOA, parameterization for a study 
area in Kisumu, Kenya was analysed. We concluded that the GSO is useful as a conceptual classification schema to serve as a basis for information extraction from VHR images. We expect that such an approach can help to remove the ambiguities in slum definition and also guide the classification process in an objective way.

\subsubsection{Research objective 2}

Determining the transferability of an OOA method to identify and classify slums using VHR satellite images.

Although the GSO is a useful framework to formalize knowledge about complex features such as slums, it may be too general to be immediately useful to parameterize an image classification for a specific setting. Thus, an intermediate step is to adapt the GSO to the specific local conditions of a city by creating a local ontology that is better tuned to what is needed for OOA ruleset development in a specific case. In chapter 3, using the GSO as a basis, we tested the transferability of an OOA method for slum classification developed on a single image area to two other image areas for the city of Ahmedabad, India. To include contextual knowledge, expert knowledge was encoded in the ontological framework to classify slums in Ahmedabad. Further, the method comprised of developing a ruleset in OOA and exploring its applicability on three subsets with varying sizes, extents and, spectral and spatial characteristics. A set of image-based parameters was identified that was used for differentiating slums from non-slum areas in all image subsets. To explore the transferability, weights were assigned to the parameters used for classification depending on the need for adaptation from one subset to another. Results show that textural features such as entropy and contrast derived from a grey level co-occurrence matrix (GLCM), and the size of image segments (Area) were stable parameters for classification of built-up areas and the identification of slums. However, the final slum classification required some additional cleaning based upon topological relationships between image segments. Relations with classified slum objects, in terms of enclosed by slums (ES) and relative border with slum (RB) have been used to refine the final slum classification. These parameters were applied to all subsets with minimal adaptation and thus, are considered as being most transferable. The analysis on three different subsets shows that final accuracies are ranging from $47 \%$ to $68 \%$.

The work presented in this study also provides a better understanding of the complexities related to slum identification and classification, especially in an Indian context. This includes the problem of differentiating between formallooking informal areas, and informal-looking formal building clusters, as these may lead to misclassification. For example, old city centres, road-side shops and markets in many Indian cities may appear similar to slum areas when 
observed from space. Additionally, the lack of reliable reference data could prove to be a hindrance for slum detection studies. However, considering these challenges and that only morphological indicators derived from images were used for slum classification, the accuracy results are acceptable. We concluded that the method produced useful results as it allowed location specific adaptation. The same basic approach and parameters can most likely be applied to a different context with local adaptation. Once the ruleset has been finalized an efficient monitoring of slums over time (e.g., size, density, changes in road network characteristics) becomes possible.

\subsubsection{Research objective 3}

Development of a hierarchical classification method in an OOA environment to detect and classify slums from VHR image.

In chapter 4, a hierarchical method for slum detection from VHR imagery based on the morphology of the built environment as expressed in generic slum ontology (GSO) is presented. Context-specific expert knowledge was integrated to form a local slum ontology suited for the city of Pune, India. Furthermore, texture and spatial metrics were calculated from a Quickbird image (red, green and blue bands) of the city. A hierarchical approach comprising the two levels of ontology, the object level (Level 1) and settlement level (Level 2), was used in an OOA environment. Our analysis identified that textural features derived from a grey level co-occurrence matrix (GLCM), contrast (GLCMCon), in addition to three bands of the image were appropriate for delineating segments of slum areas or parts thereof at the settlement level.

The Level 1 classification of basic land cover features such as built-up and vegetation were used as an input for Level 2 classification. Spatial metrics such as the size of segments, proportions of vegetation and built-up were used for slum detection. The parameters: relative area of shadow RA(S), mean green $(M G)$, distance to classified slums $D(S L)$ and enclosed by slums (ES) were useful for refining the classification. A clear difference in classification accuracy was revealed between the two ontological levels. The overall accuracy at Level 1 equal to $80.8 \%$ was remarkably high and adequate as an input to the GSO considering the limited spectral resolution of the Quickbird image. The accuracy of slum classification at the settlement level was equal to $60 \%$. This value was considered as promising, considering that a rather simple method was applied for slum identification. Our results are potentially useful for city managers especially in cities where no alternative data is available to get updates regarding slums on the ground. We concluded that the method combining the GSO, parameterization in OOA and V-I-S model has potential for successful slum detection in contexts with similar morphology. 


\subsubsection{Research objective 4}

\section{Quantification of uncertainties related to image interpretations of urban slums.}

Image interpretations can be used as reference data for accuracy assessment in OOA. This approach is also commonly used for evaluation of classified products in remote sensing. Image interpretations, however, also contain uncertainties which may negatively impact the accuracy results. Thus, there may be potential drawbacks in terms of the reliability of accuracy assessment. In chapter 5, we studied the spatial uncertainties related to the delineations of slums in three different contexts, Ahmedabad in India, Nairobi in Kenya and Cape Town in South Africa. Nineteen image interpretations and supplementary data per case were acquired by means of semi-structured questionnaires. These interpretations were combined and overlaid to study deviations in slum boundaries and to identify uncertain areas. Existential and extensional uncertainty, referring to the uncertainty related to the existence of a slum in reality and determination of boundary of slums respectively, were modeled using random sets.

This study identified the factors of the built environment that experts use to distinguish slums from non-slum areas and factors leading to deviations in slum identification in the three contexts and hence the reasons for uncertainties. In all contexts there was a considerable variation in the delineated areas suggesting that the concept slum, as used by the interpreters, is fuzzy. The level of experience of the interpreters did not significantly affect uncertainty i.e. the variation in slum maps produced by more experienced slum mappers was not significantly different from that of less experienced slum mappers. However, highly significant differences in the variability of slum extents were found between the three cities. Even after removing the more unlikely areas we found that the most likely slum areas range between 42 and $61 \%$ of the total delineated areas. The distinction between slum and non-slum areas was more evident and more clearly identifiable on the images for Nairobi and Cape Town. The more complex morphology of Ahmedabad, that is generally denser with much smaller buildings, and with a large historic city quarter which is visually similar to slum areas, resulted in a higher uncertainty of the slum interpretations. We concluded that local knowledge is important for the interpretation of slum areas, playing an even greater role in complex urban environments such as Ahmedabad. 


\subsection{Reflections}

This section reflects on the findings of the thesis. It consists of the summary of the main contributions and the recommendations for further research.

\subsubsection{Main contributions}

First of all, in the context of slum identification and mapping, a major contribution of this thesis is the conceptual framework for slums in the form of generic slum ontology (GSO). Variability in slum definitions and appearances are major obstacles in developing generically applicable slum identification methods. Previous studies mainly focused on developing methods for a particular context with limited scope for world-wide application. Further, local and regional perceptions of housing standards and social conditions can also influence the understanding of what is considered a slum. The ontological approach conceptualizes slums using indicators related to the morphology of the built environment which are flexible enough to be adapted to local conditions. The GSO is an important achievement towards an objective approach for slum mapping by providing a comprehensive description of spatial characteristics and their relationships to represent and characterize slums in an VHR satellite image.

The second major contribution of this thesis is presenting a case of applicability of GSO together with the development of an OOA method to classify slums. We explored how the ontology can bring knowledge into the OOA process in a structured and systematic manner. We particularly tested the transferability of the method on different subsets of a city resulting in a set of image parameters identified as most stable. This study presented an innovative method of utilizing expert knowledge encoded in the ontological framework as a basis for classification and thus addressing the issue of slum variability.

The third major contribution is the use of a combination of texture and spatial metrics to classify slums using a hierarchical classification in OOA. Contextual knowledge was included in the form of a local slum ontology. The study showed that abundance of built-up/impervious and low vegetation can be used as a proxies for the presence of slums and the approach has potential for successful application on contexts with similar morphology.

The final contribution of this study is the quantification of uncertainties related to slum identification. There are many potential applications of the classified slum products as presented in chapter 3 and 4 of this thesis - for example the estimation of slum populations, application in crisis management plans, in slum upgrading programs and updating of city development plans. Fitness of use of and hence the quality of remote sensing products is, however, determined by accuracy assessments. Among other factors, these assessments may be 
influenced by differences in conceptualization, image interpretation skills, and acquaintance with context. This work recognizes the importance of addressing the issue of data quality and provides an analysis of slum-related uncertainties.

\subsubsection{Recommendations for further research}

Having investigated the use of VHR imageries for slum identification and classification, the following topics may be considered for future research in the direction of slum mapping:

- The applicability of GSO should be further explored for image classification in cities of other parts of the world. Though envisaged for world-wide applicability, this thesis could provide test cases of two Indian cities for classification of slums. Applicability on different cultural spheres and urban morphologies should be tested.

- Classifying slums in larger areas is challenging as there is more variation in history and ground conditions and hence, more spatial variation. This unavoidably is likely to lead to a decrease in accuracy. Possible ways to overcome this reduction would be a hierarchical approach by splitting up the larger area in a mosaic of smaller subareas, classify those and then integrate towards the settlement level.

- Only morphological indicators derived from images were used for slum classification in this thesis. Additional information in the form of GIS layers may be helpful in refining the results further. For example, slum areas tend to have community water connections instead of individual taps in each household. If detailed data on water connections are available and can be integrated with population data of a settlement, the combination dense population with few water connections could then be used to locate probable slums. Similarly, GIS data on other utilities such as electricity or gas connections may also be useful. Such additional information may also address the problem of distinguishing formal-looking informal areas, and informal-looking formal building clusters.

- Inclusion of additional GIS layers to improve the analysis including additional information in terms of surface and terrain elevation conditions may help in improving the results. Many slums are located on steep slopes and surface height information can be used to differentiate slum buildings from formal, high-rise buildings.

- Further study of characteristics of slums in terms of patterns, size, density and their manifestations in the images may be useful. In such future research, relations with other classes, derived at the environs level of ontology may be used to refine results. 
- Research on use of other spatial metrics in combination of OOA should be explored.

- Use of the thermal band as additional information should be explored. This, specifically in areas where roofs are made of corrugated iron sheets or any other metals. The surface (roof) temperatures may tend to be higher in slums compared to other areas.

- Neighborhoods look different at different spatial scales. If an image is degraded several times, different textures will be obtained which may be quite unique for slums and non-slums. This kind of multi-scale analysis could be carried out in OOA. 
Appendix: Questionnaire for expert survey 
The following version of the questionnaire is an appendix of the second and the fifth chapter of this thesis.

\section{Slum Definition}

1. What is a slum - i.e. how do you define a slum?

2. Do you distinguish slums from other informal settlements, how?

3. Are there other types of settlements that you consider to be associated with sub-standard living conditions? If so, what are they and what makes them sub-standard?

\section{Personal experience}

4. Have you ever been to a slum or slums?

5. If yes, which city/cities, country or countries?

6. In your experience, is there much difference between slums in different contexts / countries or cities? Can you mention the names of different kind of slums in your context?

\section{Urban Morphology based Indicators for slums:}

In the following section, kindly provide information on each of the indicators based on your context. Please use knowledge of slums from your own city/country or the ones you are most familiar with. The "add more" section is for you to provide additional parameters, if any, not included in the table.

- Mention slum settlement/city name:

- Type of slum (local name, or major distinguishing factor):

- Does the slum, you are referring to, have a clear structure or chaotic structure?

- How did the slum grow over time: sudden growth or gradual growth over a long period? 


\begin{tabular}{|c|c|c|}
\hline $\begin{array}{l}\text { Indicator / } \\
\text { Description }\end{array}$ & Measure & Please specify \\
\hline \multirow{7}{*}{$\begin{array}{l}\text { Characteristic of } \\
\text { building structure }\end{array}$} & $\begin{array}{l}\text { Building type } \\
\text { ( e.g. attached, detached) }\end{array}$ & \\
\hline & Number of floors & \\
\hline & $\begin{array}{l}\text { Building footprint } \\
\text { (Specify the range of building sizes } \\
\text { typically found : } \\
<10 \mathrm{~m}^{2} \\
10-20 \mathrm{~m}^{2} \\
20-40 \mathrm{~m}^{2} \\
40-80 \mathrm{~m}^{2} \\
>80 \mathrm{~m}^{2} \text { ) }\end{array}$ & \\
\hline & $\begin{array}{l}\text { Building height } \\
\text { (approx. height in meters) }\end{array}$ & \\
\hline & $\begin{array}{l}\text { Roof material } \\
\text { ( e.g. tin, concrete) }\end{array}$ & \\
\hline & Wall material & \\
\hline & Floor material & \\
\hline
\end{tabular}

\begin{tabular}{|l|l|l|}
\hline $\begin{array}{l}\text { Indicator / } \\
\text { Description }\end{array}$ & Measure & Please specify \\
\hline \multirow{5}{*}{$\begin{array}{l}\text { Characteristic of } \\
\text { building structure }\end{array}$} & $\begin{array}{l}\text { Building type } \\
\text { ( e.g. attached, detached) }\end{array}$ & \\
\cline { 2 - 3 } & $\begin{array}{l}\text { Number of floors } \\
\text { (Specify the range of building sizes } \\
\text { typically found : } \\
\text { < } 10 \mathrm{~m}^{2} \\
10-20 \mathrm{~m}^{2} \\
20-40 \mathrm{~m}^{2} \\
40-80 \mathrm{~m}^{2} \\
>80 \mathrm{~m}^{2} \text { ) }\end{array}$ & \\
\cline { 2 - 3 } & $\begin{array}{l}\text { Building height } \\
\text { (approx. height in meters) }\end{array}$ & \\
\hline & $\begin{array}{l}\text { Roof material } \\
\text { (e.g. tin, concrete) }\end{array}$ & \\
\hline & Wall material & \\
\hline & Floor material & \\
\hline
\end{tabular}




\begin{tabular}{|l|l|l|}
\hline Indicator / Description & Measure & Please Specify \\
\hline \multirow{3}{*}{$\begin{array}{l}\text { Density (amount of built-up in } \\
\text { a settlement) }\end{array}$} & Roof Coverage (\%) & \\
\cline { 2 - 3 } & Open space (\%) & \\
\cline { 2 - 3 } & Vegetation (\%) & \\
\cline { 2 - 3 } & $\begin{array}{l}\text { Number of buildings per } \\
\text { hectare }\end{array}$ & \\
\hline
\end{tabular}

\begin{tabular}{|l|l|}
\hline Indicator / Description & $\begin{array}{l}\text { Can you specify certain shape for a slum } \\
\text { settlement, e.g. elongated, circular? Why? }\end{array}$ \\
\hline Settlement shape & \\
\hline
\end{tabular}

\begin{tabular}{|c|c|c|}
\hline Indicator & Measure & $\begin{array}{l}\text { Please circle the number that represents the site } \\
\text { condition of a slum in your context }\end{array}$ \\
\hline \multirow{5}{*}{$\begin{array}{l}\text { Site } \\
\text { condition }\end{array}$} & Flood zone & not likely $-1-----z------3------4------5--$ very likely \\
\hline & Steep slopes & not likely $-1-----z------3------4------5--$ very likely \\
\hline & $\begin{array}{l}\text { Proximity to } \\
\text { railway } \\
\text { lines, } \\
\text { highways, } \\
\text { major roads } \\
\end{array}$ & not likely $-1------2------3------4-----5--$ very likely \\
\hline & $\begin{array}{l}\text { Proximity to } \\
\text { high-voltage } \\
\text { power lines }\end{array}$ & not likely-4-----2------3-----4---- - 5-- very likely \\
\hline & $\begin{array}{l}\text { Proximity to } \\
\text { hazardous } \\
\text { industries }\end{array}$ & not likely-4-----2------3-----4---- - 5-- very likely \\
\hline
\end{tabular}




\begin{tabular}{|c|c|c|}
\hline $\begin{array}{l}\text { Indicator / } \\
\text { Description }\end{array}$ & Measure & $\begin{array}{l}\text { Please circle the number that represents the } \\
\text { neighborhood location of a slum in your context }\end{array}$ \\
\hline \multirow{4}{*}{$\begin{array}{l}\text { Neighb- } \\
\text { orhood } \\
\text { location }\end{array}$} & $\begin{array}{l}\text { Connected to } \\
\text { infrastructure } \\
\text { in neighboring } \\
\text { areas }\end{array}$ & 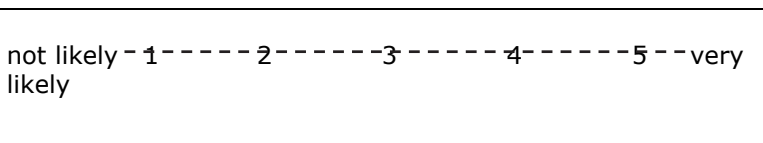 \\
\hline & $\begin{array}{l}\text { Close } \\
\text { to/inside } \\
\text { neighborhood } \\
\text { of low socio- } \\
\text { economic } \\
\text { status }\end{array}$ & $\begin{array}{l}\text { not likely- -1----- - }------3-----4------5-- \text { very } \\
\text { likely }\end{array}$ \\
\hline & $\begin{array}{l}\text { Close to } \\
\text { employment } \\
\text { (e.g. CBD, } \\
\text { industries ) }\end{array}$ & $\begin{array}{l}\text { not likely-1----- z------3------4------ 5-- very } \\
\text { likely }\end{array}$ \\
\hline & $\begin{array}{l}\text { Close to } \\
\text { neighborhood } \\
\text { of } \\
\text { middle/high } \\
\text { socio- } \\
\text { economic } \\
\text { status }\end{array}$ & $\begin{array}{l}\text { not likely-1----- z------3----- - } 4------5-\text {-very } \\
\text { likely }\end{array}$ \\
\hline
\end{tabular}

- Are there any other physical factors that you consider to be important for identifying and mapping slums?

Please have a look at the images provided to you. Identify any slum areas that you see, delineate them on Google earth and answer the following questions. The links to the corresponding. $\mathrm{kml}$ files are provided to you.

- What factors did you use to delineate boundary of slums?

- If you are unable to identify and delineate the extent, explain why?

- Please circle the number that represents how you feel about the quality of your delineation:

Very inaccurate --1----2-----3----4-----5- Very Accurate 


\section{Bibliography}

Agarwal, P. 2005. Ontological considerations in GIScience. International Journal of Geographical Information Science, 19(5), 501 - 536.

Agouris, P., Stefanidis, A., Bittner, T. and Winter, S., 1999. On Ontology in Image Analysis. Integrated Spatial Databases. Berlin / Heidelberg: Springer 168-191.

Aguilar, M., Saldaña, M. and Aguilar, F. 2013. GeoEye-1 and WorldView-2 pansharpened imagery for object-based classification in urban environments. International Journal of Remote Sensing, 34(7), 25832606.

Albrecht, F., Lang, S. and Hölbling, D. 2010. Spatial accuracy assessment of object boundaries for object-based image analysis. The International Archives of the Photogrammetry, Remote Sensing and Spatial Information Sciences, 38(4), C7.

Angeles, G., Lance, P., Barden-O'Fallon, J., Islam, N., Mahbub, A. Q. M. and Nazem, N. I. 2009. The 2005 census and mapping of slums in Bangladesh: design, select results and application. International Journal of Health Geographics, 8, 19.

Baatz, M. and Schape, A., 2000. Multiresolution segmentation: an optimization approach for high quality multi-scale image segmentation. In: Strobl, J. ed. Angewandte Geographische Informationsverarbeitung XII. Beiträge zum AGIT-Symposium. Salzburg, 12-23.

Baltsavias, E. P. 2004. Object extraction and revision by image analysis using existing geodata and knowledge: current status and steps towards operational systems. ISPRS Journal of Photogrammetry and Remote Sensing, 58(3-4), 129-151.

Baud, I., Kuffer, M., Pfeffer, K., Sliuzas, R. and Karuppannan, S. 2010. Understanding heterogeneity in metropolitan India: The added value of remote sensing data for analyzing sub-standard residential areas. International Journal of Applied Earth Observation and Geoinformation, 12(5), 359-374.

Baud, I., Pfeffer, K., Sridharan, N. and Nainan, N. 2009. Matching deprivation mapping to urban governance in three Indian mega-cities. Habitat International, 33(4), 365-377.

Baud, I., Sridharan, N. and Pfeffer, K. 2008. Mapping Urban Poverty for Local Governance in an Indian Mega-City: The Case of Delhi. Urban Studies, 45(7), 1385-1412.

Belgiu, M. and Drăgut, L. 2014. Comparing supervised and unsupervised multiresolution segmentation approaches for extracting buildings from very high resolution imagery. ISPRS Journal of Photogrammetry and Remote Sensing, 96, 67-75.

Belgiu, M., Drăgut, L. and Strobl, J. 2014a. Quantitative evaluation of variations in rule-based classifications of land cover in urban neighbourhoods using WorldView-2 imagery. ISPRS Journal of Photogrammetry and Remote Sensing, 87, 205-215.

Belgiu, M., Hofer, B. and Hofmann, P. 2014b. Coupling formalized knowledge bases with object-based image analysis. Remote Sensing Letters, 5(6), 530-538. 
Belmonte, M. V., Pérez-de-la-Cruz, J. L. and Triguero, F. 2008. Ontologies and agents for a bus fleet management system. Expert Systems with Applications, 34(2), 1351-1365.

Benslimane, D., Leclercq, E., Savonnet, M., Terrasse, M. N. and Yétongnon, K. 2000. On the definition of generic multi-layered ontologies for urban applications. Computers, Environment and Urban Systems, 24, 191214.

Benz, U. C., Hofmann, P., Willhauck, G., Lingenfelder, I. and Heynen, M. 2004. Multi-resolution, object-oriented fuzzy analysis of remote sensing data for GIS-ready information. ISPRS Journal of Photogrammetry and Remote Sensing, 58(3), 239-258.

Bhatt, M., 2003. Urban Slums Reports: The case of Ahmedabad, India. Nairobi: UN-HABITAT.

Blaschke, T. 2010. Object based image analysis for remote sensing. ISPRS Journal of Photogrammetry and Remote Sensing, 65, 2-16.

Blaschke, T., Lang, S. M. and Hay, G. J., 2008. Object based image analysis : spatial concepts for knowledge - driven remote sensing applications Berlin: Springer.

Campari, I., 1996. Uncertain boundaries in urban space. In: Burrough, P. and Frank, A. eds. Geographic objects with indeterminate boundaries. Britain: Taylor \& Francis Ltd, 57-69.

Champagne, C., McNairn, H., Daneshfar, B. and Shang, J. 2014. A bootstrap method for assessing classification accuracy and confidence for agricultural land use mapping in Canada. International Journal of Applied Earth Observation and Geoinformation, 29, 44-52.

Chandrasekaran, B., Josephson, J. R. and Benjamins, V. R. 1999. What are ontologies, and why do we need them? IEEE Intelligent Systems, 14(1), 20-26.

Cleve, C., Kelly, M., Kearns, F. R. and Moritz, M. 2008. Classification of the wildland-urban interface: A comparison of pixel- and object-based classifications using high-resolution aerial photography. Computers, Environment and Urban Systems, 32(4), 317-326.

COI, 2001. Census of India [online]. Office of the Registrar General and Census Commissioner, Government of India. Available from: http://www.censusindia.gov.in/ [Accessed 24 June, 2011].

COI, 2011. Census of India [online]. Office of the Registrar General and Census Commissioner, Government of India. Available from: http://www.censusindia.gov.in/ [Accessed 24 June, 2011].

Congalton, R. 1991. A review of assessing the accuracy of classifications of remotely sensed data. Remote Sensing of Environment, 37(1), 35-46.

Congalton, R. and Mead, R. A. 1983. A quantitative method to test for consistency and correctness in photointerpretation. Photogrammetric Engineering \& Remote Sensing, 49(1), 69-74.

Couclelis, H., 1996. Towards an operational typology of geographic entities with ill-defined boundaries. In: Burrough, P. and Frank, A. eds. Geographic objects with indeterminate boundaries. Britain: Taylor \& Francis Ltd, 45-55.

Davis, M., 2006. Planet of Slums. London: Verso.

Drăguţ, L., Tiede, D. and Levick, S. R. 2010. ESP: a tool to estimate scale parameter for multiresolution image segmentation of remotely sensed 
data. International Journal of Geographical Information Science, 24(6), 859-871.

Dubovyk, O., Sliuzas, R. and Flacke, J. 2011. Spatio-temporal modelling of informal settlement development in Sancaktepe district, Istanbul, Turkey. ISPRS Journal of Photogrammetry and Remote Sensing, 66(2), 235-246.

Ebert, A., Kerle, N. and Stein, A. 2009. Urban social vulnerability assessment with physical proxies and spatial metrics derived from air- and spaceborne imagery and GIS data. Natural Hazards, 48(2), 275-294.

Edwards, G. and Lowell, K. 1996. Modeling uncertainty in photointerpreted boundaries. Photogrammetric engineering and remote sensing, 62(4), 377-390.

Fan, Z. and Zlatanova, S., 2010. Exploring ontology potential in emergency management. Proceedings of the Gi4DM conference- Geomatics for disaster management Torino, Italy, 1-6.

Fernández López, M., Gómez-Pérez, A., Sierra, J. P. and Sierra, A. P. 1999. Building a Chemical Ontology Using Methontology and the Ontology Design Environment. IEEE Intelligent Systems, 14(1), 37-46.

Fonseca, F. T., Egenhofer, M. J., Davis, C. A. and Borges, K. A. V. 2000. Ontologies and knowledge sharing in urban GIS. Computers, Environment and Urban Systems, 24(3), 251-272.

Foody, G. M. and Boyd, D. S. 2013. Using volunteered data in land cover map validation: Mapping West African forests. IEEE journal of Selected Topics in Applied Earth Observations and Remote Sensing, 6(3), 13051312.

Gasevic, D., Djuric, D. and Devedzic, V., 2009. Model driven engineering and ontology development. Berlin: Springer.

Gruber, T. R. 1993. A translation approach to ontology specifications. Knowledge Acquisition, 5(2), 199-220.

Guarino, N. 1995. Formal ontology, conceptual analysis and knowledge representation. International Journal of Human-Computer Studies, 43(5), 625-640.

Guarino, N. 1997. Understanding, building and using ontologies. International Journal of Human-Computer Studies, 46(2-3), 293-310.

Haala, N. and Brenner, C. 1999. Extraction of buildings and trees in urban environments. ISPRS Journal of Photogrammetry and Remote Sensing, 54(2-3), 130-137.

Hall, G. B., Malcolm, N. W. and Piwowar, J. M. 2001. Integration of Remote Sensing and GIS to Detect Pockets of Urban Poverty: The Case of Rosario, Argentina. Transactions in GIS, 5(3), 235-253.

Haralick, R. M., Shanmugam, K. and Dinstein, I. H. 1973. Textural Features for Image Classification. IEEE Transactions on Systems, Man and Cybernetics, 3, 610-621.

Hasan, A. 2006. Orangi Pilot Project: the expansion of work beyond Orangi and the mapping of informal settlements and infrastructure. Environment and Urbanization, 18(2), 451-480.

Herold, M., Liu, X. and Clarke, K. C. 2003. Spatial metrics and image texture for mapping urban land use. Photogrammetric Engineering \& Remote Sensing, 69(9), 991-1001.

Herold, M., Mayaux, P., Woodcock, C. E., Baccini, A. and Schmullius, C. 2008. Some challenges in global land cover mapping: An assessment of 
agreement and accuracy in existing $1 \mathrm{~km}$ datasets. Remote Sensing of Environment, 112(5), 2538-2556.

Herold, M., Scepan, J. and Clarke, K. C. 2002. The use of remote sensing and landscape metrics to describe structures and changes in urban land uses. Environment and Planning A, 34(8), 1443-1458.

Hofmann, P., 2001. Detecting Informal Settlements from Ikonos Image Data Using Methods of Object Oriented Image Analysis-An Example from Cape Town (South Africa) In: Jürgens, C. ed. Remote Sensing of Urban Areas. 41-42.

Hofmann, P., Blaschke, T. and Strobl, J. 2011. Quantifying the robustness of fuzzy rule sets in object-based image analysis. International Journal of Remote Sensing, 32(22), 7359-7381.

Hofmann, P., Strobl, J., Blaschke, T. and Kux, H., 2008. Detecting informal settlements from Quickbird data in Rio De Janeiro using an object based approach. In: Blaschke, T., Lang, S. and Hay, G. J. eds. ObjectBased Image Analysis. Berlin: Springer, 531-553.

$\mathrm{Hu}, \mathrm{X}$. and Weng, Q. 2010. Impervious surface area extraction from IKONOS imagery using an object-based fuzzy method. Geocarto International, 26(1), 3-20.

Jain, S. 2007. Use of Ikonos satellite data to identify informal settlements in Dehradun, India. International Journal of Remote Sensing, 28(15), 3227-3233.

Jain, S., Sokhi, B. S. and Sur, U. 2005. Slum Identification Using Highresolution Satellite Data. GIM International, 19(9), 60.

Joshi, P., Sen, S. and Hobson, J. 2002. Experiences with surveying and mapping Pune and Sangli slums on a geographical information system (GIS). Environment and Urbanization, 14(2), 225-240.

Karanja, I. 2010. An enumeration and mapping of informal settlements in Kisumu, Kenya, implemented by their inhabitants. Environment and Urbanization, 22(1), 217-239.

Kit, O. and Lüdeke, M. 2013. Automated detection of slum area change in Hyderabad, India using multitemporal satellite imagery. ISPRS Journal of Photogrammetry and Remote Sensing, 83, 130-137.

Kit, O., Lüdeke, M. and Reckien, D. 2012. Texture-based identification of urban slums in Hyderabad, India using remote sensing data. Applied Geography, 32(2), 660-667.

Kohli, D., Sliuzas, R., Kerle, N. and Stein, A. 2012. An ontology of slums for image-based classification. Computers, Environment and Urban Systems, 36(2), 154-163.

Kohli, D., Warwadekar, P., Kerle, N., Sliuzas, R. and Stein, A. 2013. Transferability of Object-Oriented Image Analysis Methods for Slum Identification. Remote Sensing, 5(9), 4209-4228.

Kombe, W. J. 2005. Land use dynamics in peri-urban areas and their implications on the urban growth and form: the case of Dar es Salaam, Tanzania. Habitat International, 29(1), 113-135.

Kuffer, M., Barros, J. and Sliuzas, R. V. 2014. The development of a morphological unplanned settlement index using very-high-resolution (VHR) imagery. Computers, Environment and Urban Systems, 48, 138152. 
Lemma, T., Sliuzas, R. V. and Kuffer, M., 2006. A participatory approach to monitoring slum conditions: an example from Ethiopia. Participatory Learning and Action. 54-58.

Lepczyk, C. A., Lortie, C. J. and Anderson, L. J. 2008. An ontology for landscapes. Ecological Complexity, 5(3), 272-279.

Li, X., Liu, G., Ling, A., Zhan, J., An, N., Li, L. and Sha, Y., 2008. Building a Practical Ontology for Emergency Response Systems. Proceedings of the 2008 International Conference on Computer Science and Software Engineering. Wuhan, China: IEEE, 222-225.

Lu, D. S. and Weng, Q. H. 2005. Urban classification using full spectral information of Landsat ETM+ imagery in Marion County, Indiana. Photogrammetric engineering and remote sensing, 71(11), 12751284.

Lunetta, R. S., Liames, J., Knight, J., Congalton, R. G. and Mace, T. H. 2001. An assessment of reference data variability using a virtual field reference database. Photogrammetric engineering and remote sensing, 67(6), 707-715.

Martha, T., Kerle, N., Westen, C., Jetten, V. and Kumar, V. 2011. Segment optimization and data-driven thresholding for knowledge-based landslide detection by object-based image analysis. IEEE Transactions on Geoscience and Remote Sensing, 49(12), 4928-4943.

Martha, T., Kerle, N., Westen, C., Jetten, V. and Kumar, V. 2012. Objectoriented analysis of multi-temporal panchromatic images for creation of historical landslide inventories. ISPRS Journal of Photogrammetry and Remote Sensing, 67, 105-119.

Martínez, J. 2009. The use of GIS and indicators to monitor intra-urban inequalities. A case study in Rosario, Argentina. Habitat International, 33(4), 387-396.

MASHAL, 2011. Pune city slum atlas. Pune: Maharashtra Social Housing and Action League.

Mason, S. O. and Fraser, C. S. 1998. Image Sources for Informal Settlement Management. The Photogrammetric Record, 16(92), 313-330.

Matei, O. 2008. Ontology-Based Knowledge Organization for the Radiograph Images Segmentation. Advances in Electrical and Computer Engineering, 8(1), 56-61.

McGill, R. 1995. Urban management performance: an assessment framework for Third World city managers. Cities, 12(5), 337-351.

Molenaar, M., Three conceptual uncertainty levels for spatial objects. ed. GIS Fundamentals and Spatial Databases, 2000 Amsterdam: International archives of photogrammetry and remote sensing, 670-677.

Myint, S. W., Galletti, C. S., Kaplan, S. and Kim, W. K. 2013. Object vs. pixel: a systematic evaluation in urban environments. Geocarto International, 28(7), 657-678.

Myint, S. W., Gober, P., Brazel, A., Grossman-Clarke, S. and Weng, Q. 2011. Per-pixel vs. object-based classification of urban land cover extraction using high spatial resolution imagery. Remote Sensing of Environment, 115(5), 1145-1161.

Neuwirth, R., 2005. Shadow cities : a billion squatters, a new urban world. New York: Routledge.

Niebergall, S., Loew, A. and Mauser, W., 2007. Object-Oriented Analysis of Very High-Resolution QuickBird Data for Mega City Research in 
Delhi/India. Urban Remote Sensing Joint Event. Paris, France: IEEE, 18.

Nobrega, R. A. A., O'Hara, C. G. and Quintanilha, J. A., 2008. An object-based approach to detect road features for informal settlements near Sao Paulo, Brazil. In: Blaschke, T., Lang, S. and Hay, G. J. eds. ObjectBased Image Analysis. Berlin/Heidelberg: Springer 589-607.

Novack, T. and Kux, H. J. H. 2010. Urban land cover and land use classification of an informal settlement area using the open-source knowledge-based system InterIMAGE. Journal of Spatial Science, 55(1), 23-41.

OED, 2011. Oxford English Dictionary. Oxford: Oxford University Press.

Oleire-Oltmanns, S., Coenradie, B. and Kleinschmit, B. 2011. An Object-Based Classification Approach for Mapping Migrant Housing in the MegaUrban Area of the Pearl River Delta (China). Remote Sensing, 3(8), 1710-1723.

Ooi, G. and Phua, K. 2007. Urbanization and Slum Formation. Journal of Urban Health, 84, 27-34.

Paar, P. and Rekittke, J. 2011. Low-Cost Mapping and Publishing Methods for Landscape Architectural Analysis and Design in Slum-Upgrading Projects. Future Internet, 3(4), 228-247.

Patino, J. E. and Duque, J. C. 2013. A review of regional science applications of satellite remote sensing in urban settings. Computers, Environment and Urban Systems, 37, 1-17.

PMC, 2012. Integrated ward level disaster management plan. Pune: Pune Municipal corporation.

Pontius, R. G. and Millones, M. 2011. Death to Kappa: birth of quantity disagreement and allocation disagreement for accuracy assessment. International Journal of Remote Sensing, 32(15), 4407-4429.

Ridd, M. K. 1995. Exploring a V-I-S (vegetation-impervious surface-soil) model for urban ecosystem analysis through remote sensing: comparative anatomy for cities. International Journal of Remote Sensing, 16(12), $2165-2185$.

Salehi, B., Zhang, Y., Zhong, M. and Dey, V. 2012. Object-Based Classification of Urban Areas Using VHR Imagery and Height Points Ancillary Data. Remote Sensing, 4(8), 2256-2276.

Scepan, J. 1999. Thematic validation of high-resolution Global Land-Cover Data Sets. Photogrammetric engineering and remote sensing, 65(9), 1051-1060.

Sen, S., Hobson, J. and Joshi, P. 2003. The Pune Slum Census: creating a socio-economic and spatial information base on a GIS for integrated and inclusive city development. Habitat International, 27(4), 595-611.

Shekhar, S., 2010. Applying GIS and RS for modeling the growth of slums in Pune City, Maharashtra, India. Thesis (M.Sc.). University of Salzburg.

Sliuzas, R., Mboup, G. and Sherbinin, A., 2008a. Expert Group Meeting on Slum Identification and Mapping. Enschede, The Netherlands: ITC.

Sliuzas, R. V., 2004. Managing informal settlements : a study using geoinformation in Dar es Salaam, Tanzania. PhD Dissertation. Utrecht University, ITC Publication Series No. 112.

Sliuzas, R. V., Kerle, N. and Kuffer, M., 2008b. Object-oriented mapping of urban poverty and deprivation. Proceedings of the 4th EARSeL (European Association of Remote Sensing Laboratories) worshop on 
remote sensing for developing countries in conjunction with GISDECO 8. Istanbul, Turkey.

Sliuzas, R. V. and Kuffer, M., 2008. Analysing the spatial heterogeneity of poverty using remote sensing : Typology of poverty areas using selected RS based indicators. In: Jurgens, C. ed. Remote sensing : new challenges of high resolution - EARSeL workshop. Bochum, Germany.

Sowa, J. F., 2000. Knowledge Representation : Logical, Philosophical and Computational Foundations. Pacific Grove, CA: Brooks Cole

Stein, A., Hamm, N. A. S. and Qinghua, Y. 2009. Handling uncertainties in image mining for remote sensing studies. International Journal of Remote Sensing, 30(20), 5365-5382.

Stoler, J., Daniels, D., Weeks, J. R., Stow, D. A., Coulter, L. L. and Finch, B. K. 2012. Assessing the Utility of Satellite Imagery with Differing Spatial Resolutions for Deriving Proxy Measures of Slum Presence in Accra, Ghana. GIScience \& Remote Sensing, 49(1), 31-52.

Stow, D., Lopez, A., Lippitt, C., Hinton, S. and Weeks, J. 2007. Object-based classification of residential land use within Accra, Ghana based on QuickBird satellite data. International Journal of Remote Sensing, 28(22), 5167-5173.

Stow, D. A., Lippitt, C. D. and Weeks, J. R. 2010. Geographic object-based delineation of neighborhoods of Accra, Ghana using QuickBird satellite imagery. Photogrammetric engineering and remote sensing, 76(8), 907-914.

Swartout, W. and Tate, A. 1999. Guest Editors' Introduction: Ontologies. IEEE Intelligent Systems, 14(1), 18-19.

Taubenböck, H. and Kraff, N. J. 2014. The physical face of slums: a structural comparison of slums in Mumbai, India, based on remotely sensed data. Journal of Housing and the Built Environment, 29(1), 15-38.

Teller, J., Lee, J., Roussey, C. and Laurini, R., 2007. Pre-consensus Ontologies and Urban Databases. Ontologies for Urban Development. Springer Berlin / Heidelberg, 27-36.

Trimble, 2014. Reference Book. eCognition Developer 9.0. München, Germany.

UN-HABITAT, 2003a. The Challenge of Slums: Global Report on Human Settlements. UK and USA: United Nations Human Settlements Programme

UN-HABITAT, 2003b. Guide to Monitoring Target 11: Improving the lives of 100 million slum dwellers. Nairobi, Kenya: United Nations Human Settlements Programme.

UN-HABITAT, 2003c. Slums of the World : The face of urban poverty in the new millennium. Nairobi, Kenya: United Nations Human Settlements Programme.

UN-HABITAT, 2005. Situation analysis of informal settlements in Kisumu. Nairobi, Kenya: United Nations Human Settlements Programme.

UN-HABITAT, 2006. Analytical Perspective of Pro-poor Slum Upgrading Frameworks. Nairobi, Kenya: United Nations Human Settlements Programme.

UN-HABITAT, 2010. State of World's Cities 2010/2011. UK and USA: United Nations Human Settlements Programme.

UN-HABITAT, 2012. State of World's Cities 2012/2013. Nairobi, Kenya: United Nations Human Settlements Programme. 
UN-HABITAT, 2014. Streets as Tools for Urban Transformation in Slums : A Street-Led Approach to Citywide Slum Upgrading. Nairobi, Kenya: United Nations Human Settlements Programme.

UNESCO, 2011. Historic City of Ahmedabad [online]. Available from: http://whc.unesco.org/en/tentativelists/5616/ [Accessed 27 July, 2013].

Van Coillie, F. M. B., Gardin, S., Anseel, F., Duyck, W., Verbeke, L. P. C. and De Wulf, R. R. 2014. Variability of operator performance in remotesensing image interpretation: the importance of human and external factors. International Journal of Remote Sensing, 35(2), 754-778.

Walker, J. S. and Blaschke, T. 2008. Object-based land-cover classification for the Phoenix metropolitan area: optimization vs. transportability. International Journal of Remote Sensing, 29(7), 2021-2040.

Weeks, J., Hill, A., Stow, D., Getis, A. and Fugate, D. 2007. Can we spot a neighborhood from the air? Defining neighborhood structure in Accra, Ghana. GeoJournal, 69(1), 9-22.

Winter, S. 2001. Ontology: buzzword or paradigm shift in GI science? International Journal of Geographical Information Science, 15(7), 587590.

Zhao, X., Stein, A. and Chen, X. 2010. Application of random sets to model uncertainties of natural entities extracted from remote sensing images. Stochastic Environmental Research and Risk Assessment, 24(5), 713723.

Zhou, L. and Stein, A. 2013. Application of random sets to model uncertainty of road polygons extracted from airborne laser points. Computers, Environment and Urban Systems, 41, 289-298. 


\section{Summary}

The proliferation of slums in cities in developing countries is a major concern for local, national and international organizations. Slums are directly related to poverty. Therefore, consistent information on their nature and extent is useful for planning, implementing and monitoring urban poverty alleviation programs. Systematic quantification of slums requires spatial statistical methods for a consistent identification. This thesis aimed at developing and testing remote sensing (RS) based methods for identifying and classifying slum areas using very high resolution (VHR) satellite images. For this purpose, four studies were conducted addressing various aspects of slum mapping using RS.

Variability in slum definitions and appearances are major obstacles in developing generically applicable slum identification methods. To address this, the first study demonstrated the conceptualization of slums for identification and delineation with VHR satellite images, taking UN-HABITAT's definition of a slum household as a basis. The focus was to identify physical aspects derivable by means of RS to distinguish slums from non-slum areas using input from fifty domain-experts, covering sixteen different countries. The resulting Generic Slum Ontology (GSO) comprised concepts identified at three spatial levels: the environs level, the settlement level and the object level. The GSO serves as a comprehensive basis for image-based classification of slums, in particular, using object-oriented image analysis techniques (OOA).

The second study used the GSO as a basis and tested the transferability of an OOA method for slum classification from a single image area to two other image areas. Expert knowledge was included to classify slums in the city of Ahmedabad, India. A set of image-based parameters, that worked on all three image areas with minor adaptation, was identified as stable for differentiating slums from non-slum areas. The analysis on three image areas showed that the method produced useful results as it allowed location specific adaptation.

The third study demonstrated the implementation of a segmentation and hierarchical classification approach using OOA and GSO to detect slums in the Quickbird image of Pune city, India. Results showed that a combination of texture and spatial metrics can be used for delineating segments representing slum areas and classifying slum settlements respectively. The method followed a rather simple approach and is potentially useful for application on urban contexts with a comparable morphology.

The fourth study focused on spatial uncertainties related to the slum interpretations and delineations, in three different contexts, performed by nineteen respondents, all of whom were a part of the expert group which was the basis for the GSO. The percentage of slum area with a high level of 
agreement and the uncertain area were determined. Additionally, existential and extensional uncertainty of slums was modelled using random sets and a bootstrapped sampling method. The results were used to identify the factors of the built environment that experts use to distinguish slums from non-slum areas and also study the factors resulting in uncertainties in slum identification. The study suggests that the complexity of the urban morphology in a given city is more important for slum interpretation than professional expertise.

To conclude, this research covered various aspects of RS-based slum mapping and highlighted the opportunities and limitations of respective approaches. Starting with the ontological framework, development of advanced image analysis methods for slum classification and ending with the quantification of uncertainty in slum interpretations, the four studies integrated issues that received little attention in the past. This thesis has shown that RS data combined with spatial statistical methods are indispensable to make a solid quantitative analysis of slum developments worldwide. The various methods presented serve as a basis for further research in related fields. 


\section{Samenvatting}

De proliferatie van sloppenwijken in steden in ontwikkelingslanden is een grote zorg voor lokale, nationale en internationale organisaties. Sloppenwijken zijn direct gerelateerd aan armoede. Om die reden is het belangrijk om consistente informatie over hun aard en omvang te hebben ten behoeve van de planning, uitvoering en monitoring van bestrijdingsprogramma's voor stedelijke armoede. Een systematische kwantificering van sloppenwijken vereist ruimtelijke statistische methoden voor een consistente identificatie. Dit proefschrift richt zich op het ontwikkelen en testen van methoden gebaseerd op remote sensing (RS) voor het identificeren en classificeren van sloppenwijken vanaf zeer hoge resolutie (VHR) satellietbeelden. Hiervoor zijn vier studies uitgevoerd die diverse aspecten van sloppenwijken in kaart brengen met behulp van RS.

Variabiliteit in de definities en het voorkomen van sloppenwijk zijn belangrijke obstakels bij de ontwikkeling van generiek toepasbare identificatiemethoden voor sloppenwijken. Om dit aan te pakken, is allereerst onderzoek uitgevoerd naar de conceptualisering van sloppenwijken zoals ze kunnen worden geïdentificeerd en afgebakend vanaf VHR satellietbeelden. Hierbij is de UNHABITAT definitie van een huishouden in een sloppenwijk als basis gebruikt. De nadruk ligt op de fysieke aspecten die door middel van RS kunnen worden bepaald en die helpen om sloppenwijken van niet-sloppenwijken te onderscheiden. Hierbij is input van vijftig domein-experts uit zestien verschillende landen gebruikt. De resulterende Generic Slum Ontology (GSO) bestaat uit concepten die zijn op drie ruimtelijke niveaus zijn gedefinieerd: het omgevingsniveau, het nederzettingsniveau en het object niveau. De GSO dient als een basis voor een classificatie op basis van beelden van de sloppenwijken waarbij in het bijzonder gebruik wordt gemaakt van objectgeoriënteerde beeldanalyse-technieken (= Object Oriented Analys, OOA).

De tweede studie gebruikte de GSO als basis en testte de overdraagbaarheid van de parameters die bij een OOA classificatie zijn gebruikt voor een sloppenwijk in één gebied naar sloppenwijken in twee andere delen van het beeld. Expert kennis is meegenomen om sloppenwijken te classificeren in de stad Ahmedabad, India. Een verzameling beeld specifieke parameters die op alle drie de delen van het beeld met een kleine aanpassing werkte, is als 'stabiel' geïdentificeerd om sloppenwijken te onderscheiden van nietsloppenwijken. De analyse van de drie delen van het beeld liet zien dat de methode bruikbare resultaten opleverde omdat het lokaal-specifieke aanpassingen toestond.

De derde studie richtte zich op de implementatie van een segmentatie en een hiërarchische classificatie met behulp van OOA en GSO om sloppenwijken 
vanaf een Quickbird beeld van de stad Poona, India te detecteren. De resultaten toonden aan dat een combinatie van structuur elementen en ruimtelijke metrieken kan worden gebruikt voor de afbakening van segmenten die sloppenwijken representeren of classificeren. De gevolgde methode is een vrij eenvoudige benadering en is potentieel bruikbaar voor toepassingen binnen stedelijke contexten met een vergelijkbare morfologie.

De vierde studie richtte zich op ruimtelijke onzekerheden met betrekking tot interpretaties en afbakeningen van sloppenwijken in drie verschillende contexten, uitgevoerd door negentien respondenten. Zij behoorden allen tot de groep van deskundigen, die ook aan de basis stonden voor de GSO. Het percentage van de sloppenwijk met een hoge mate van overeenstemming en de onzekere omgeving ervan werden bepaald. Bovendien zijn existentiële en extensionele onzekerheden van sloppenwijken gemodelleerd met behulp van random sets en een bootstrap sampling methode. De resultaten zijn gebruikt om de factoren van de gebouwde omgeving vast te stellen die experts gebruiken om sloppenwijken van niet-sloppenwijken te onderscheiden en om de factoren te bestuderen waardoor onzekerheden optreden in de identificatie van sloppenwijken. De studie laat zien dat de complexiteit van de stedelijke morfologie in een bepaalde stad van meer belang is voor interpretatie van sloppenwijken dan professionele deskundigheid.

Tot slot omvat dit onderzoek een studie naar de verschillende aspecten die bij het in kaart brengen van een sloppenwijk met op RS-gebaseerde methoden een rol spelen. De resultaten wijzen erop dat de de respectieve benaderingen zowel mogelijkheden als beperkingen kennen. Dit begint bij het ontologische kader en loopt via de ontwikkeling van geavanceerde beeldanalyse methoden voor de classificatie van sloppenwijken naar de kwantificering van de onzekerheid in de interpretaties van sloppenwijken. Op deze manier hebben de vier studies verschillende onderwerpen geïntegreerd die in het verleden weinig aandacht gekregen.

Dit proefschrift heeft aangetoond dat RS gegevens gecombineerd met ruimtelijke statistische methoden onontbeerlijk zijn om een goedonderbouwde kwantitatieve analyse te maken van ontwikkelingen in sloppenwijken wereldwijd. De verschillende gepresenteerde methoden dienen als basis voor verder onderzoek binnen gerelateerde onderwerpen. 


\section{Author's Biography}

I received Masters in Geoinformatics in 2007 under the collaboration program between ITC and Indian Institute of Remote Sensing, India. My thesis involved a dynamic analysis of spatial and temporal patterns of land cover change in an urban environment, considering a combination of spatial metrics and gradient modeling. From 2007 to 2009, I was employed as a Technical Associate with the Forest Survey of India (www.fsi.org.in) in Dehradun. I worked on different projects involving GIS and remote

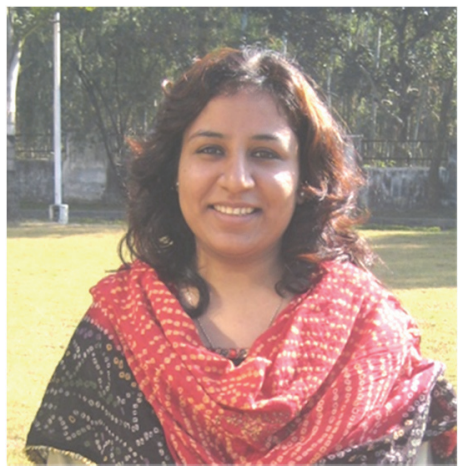
sensing techniques for assessment of forest resources in India. It was during my masters' thesis research that I developed interest in urban remote sensing. To study further in the field, in 2009, I applied and was awarded grant to pursue PhD at ITC under the Erasmus Mundus scholarship program. My PhD research aimed at developing and testing earth observation techniques for identifying and classifying slums in a variety of contexts. This research has culminated in the form of this thesis and the following publications:

Kohli, D., Sliuzas, R., Kerle, N., Stein, A., 2012. An ontology of slums for image-based classification. Computers, Environment and Urban Systems 36, 154-163.

Kohli, D., Warwadekar, P., Kerle, N., Sliuzas, R., Stein, A. 2013. Transferability of object-oriented image analysis methods for slum identification. Remote Sensing, 5(9), 4209-4228.

Kohli, D., Sliuzas, R., Stein, A. 2013. Urban slum detection using texture and spatial metrics derived from satellite imagery. Journal of Spatial Science (Undergoing revision).

Kohli, D., Stein, A., Sliuzas, R. 2013. Uncertainty analysis for image interpretations of urban slums (Submitted to Journal of Computers, Environment and Urban Systems).

Kohli, D. (2013). Spatial metrics and image texture for slum detection. NAERUS XIV / GISDECO Conference, 12-14 September, 2013. Enschede: NAERUS.

Kohli, D., Kerle, N. and Sliuzas, R.V. (2012) Local ontologies for object - based slum identification and classification. In: Proceedings of GEOBIA 2012, the 4th Geobia: Geographic object - based image analysis, May 7-9, 2012 Rio de 
Janeiro Brazil. São José dos Campos, Brazilian National Institute for Space Research (INPE), 2012. ISBN 978-85-17-00059-1 pp. 201-205.

Kohli, D., Sliuzas, R.V. and Kerle, N. (2010) Ontology of slums as a basis for object - oriented image classification. Presented at Earsel Joint SIG Workshop: Urban - 3D - Radar - Thermal Remote Sensing and Developing Countries, 2224 September 2010, Ghent, Belgium. 8 p. 


\section{ITC Dissertation List}

http://www.itc.nl/research/phd/phd graduates.aspx 